\title{
Load Flexibility Analysis of Residential HVAC and Water Heating and Commercial Refrigeration
}

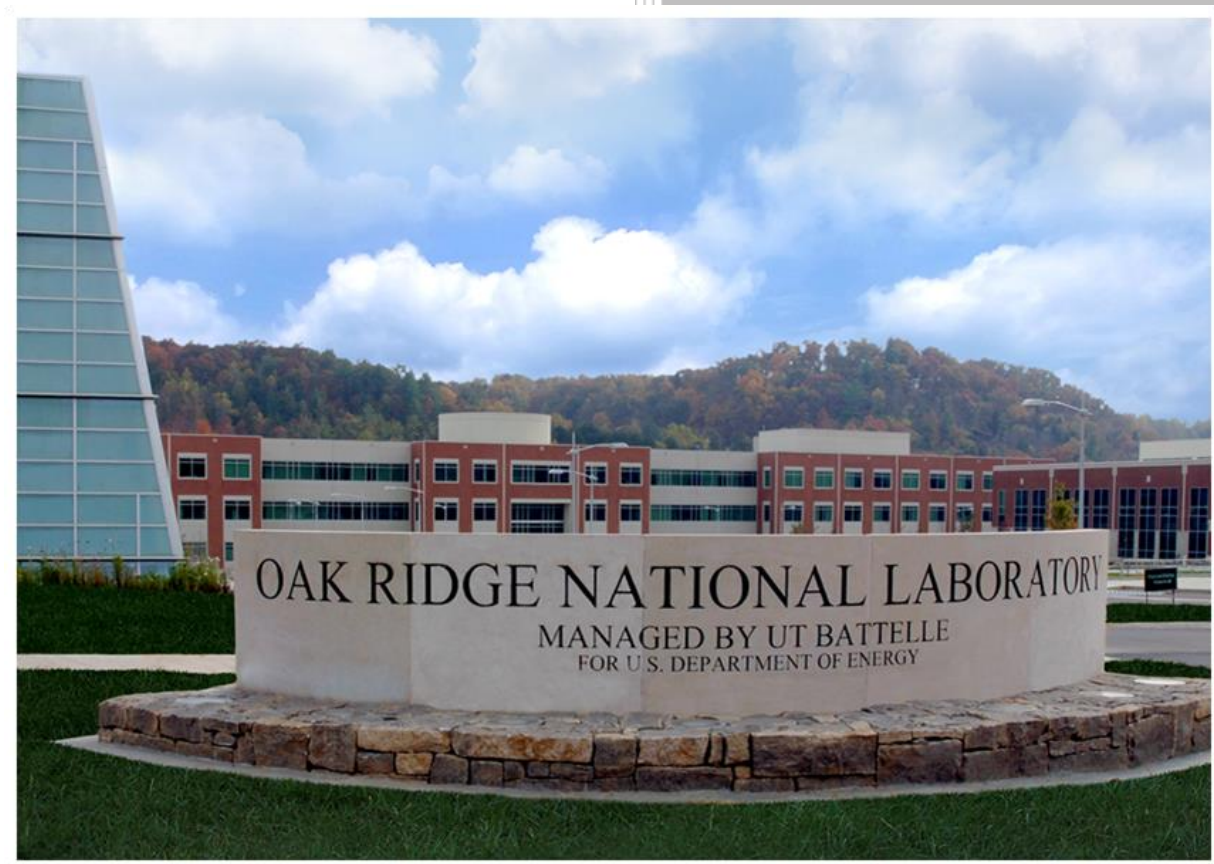

Borui Cui Jaewan Joe Jeffrey Munk Jian Sun

Teja Kuruganti

September 2019 


\section{DOCUMENT AVAILABILITY}

Reports produced after January 1, 1996, are generally available free via US Department of Energy (DOE) SciTech Connect.

Website www.osti.gov

Reports produced before January 1, 1996, may be purchased by members of the public from the following source:

National Technical Information Service

5285 Port Royal Road

Springfield, VA 22161

Telephone 703-605-6000 (1-800-553-6847)

TDD 703-487-4639

Fax 703-605-6900

E-mail info@ntis.gov

Website http://classic.ntis.gov/

Reports are available to DOE employees, DOE contractors, Energy Technology Data Exchange representatives, and International Nuclear Information System representatives from the following source:

Office of Scientific and Technical Information

PO Box 62

Oak Ridge, TN 37831

Telephone 865-576-8401

Fax 865-576-5728

E-mail reports@osti.gov

Website http://www.osti.gov/contact.html

This report was prepared as an account of work sponsored by an agency of the United States Government. Neither the United States Government nor any agency thereof, nor any of their employees, makes any warranty, express or implied, or assumes any legal liability or responsibility for the accuracy, completeness, or usefulness of any information, apparatus, product, or process disclosed, or represents that its use would not infringe privately owned rights. Reference herein to any specific commercial product, process, or service by trade name, trademark, manufacturer, or otherwise, does not necessarily constitute or imply its endorsement, recommendation, or favoring by the United States Government or any agency thereof. The views and opinions of authors expressed herein do not necessarily state or reflect those of the United States Government or any agency thereof. 
Energy and Transportation Science Division

\title{
LOAD FLEXIBILITY ANALYSIS OF RESIDENTIAL HVAC AND WATER HEATING AND COMMERCIAL REFRIGERATION
}

\author{
Borui Cui \\ Jaewan Joe \\ Jeffrey Munk \\ Jian Sun \\ Teja Kuruganti
}

Date Published: September 2019

Prepared by

OAK RIDGE NATIONAL LABORATORY

Oak Ridge, TN 37831-6283

managed by

UT-BATTELLE, LLC

for the

US DEPARTMENT OF ENERGY

under contract DE-AC05-00OR22725 



\section{CONTENTS}

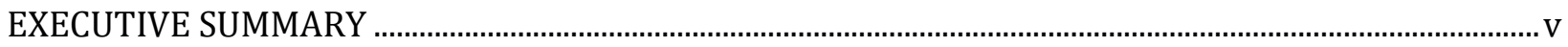

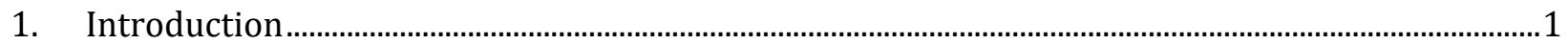

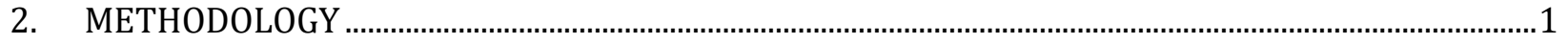

2.1 MODELING APPROACH

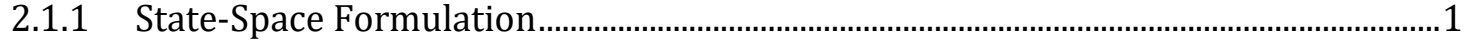

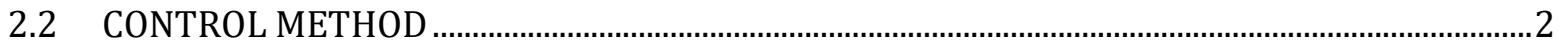

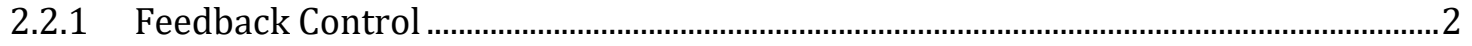

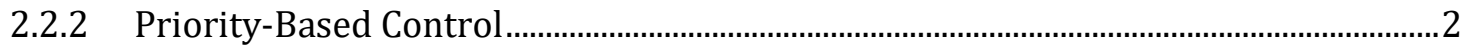

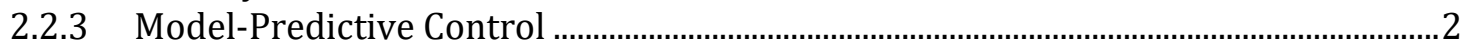

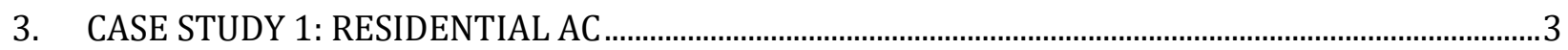

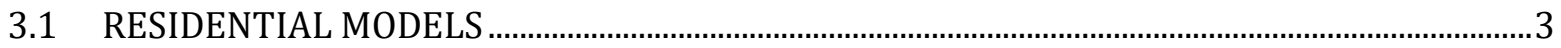

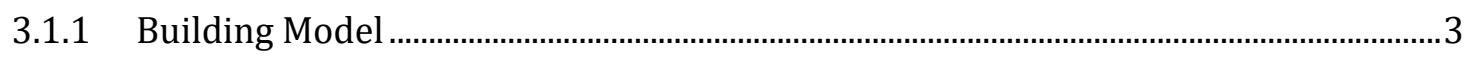

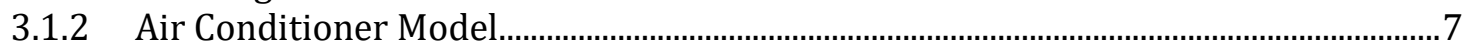

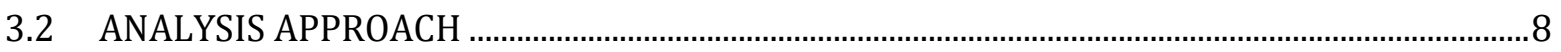

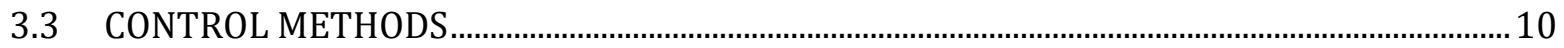

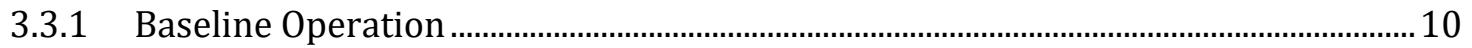

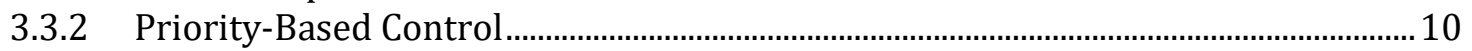

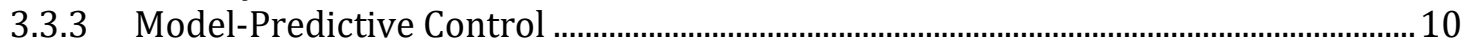

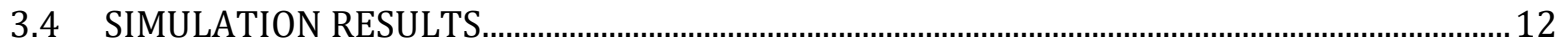

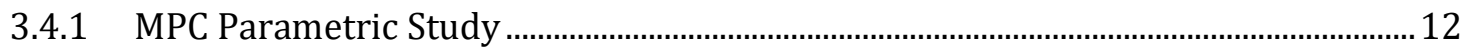

3.4.2 Hourly Load Flexibility with Demand Charge Analysis.................................................14

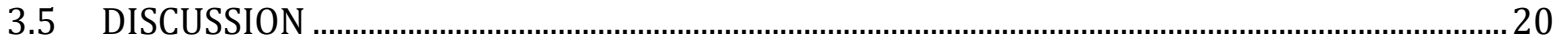

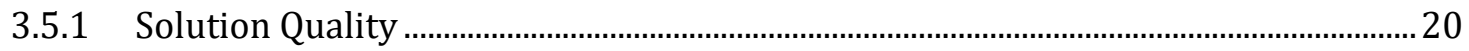

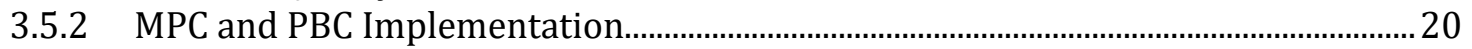

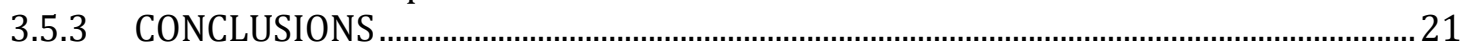

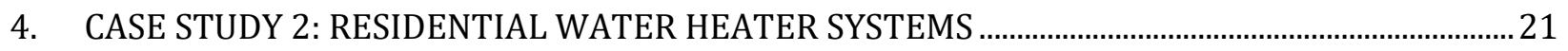

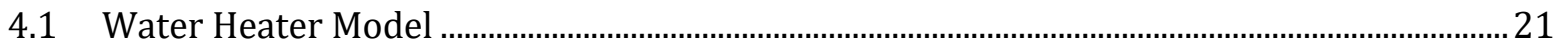

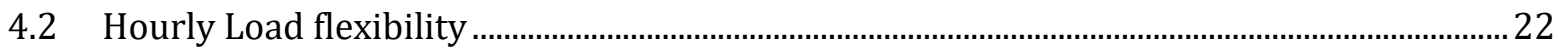

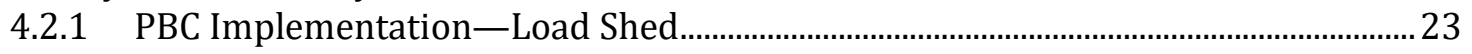

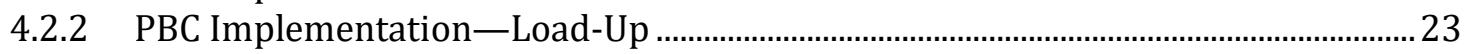

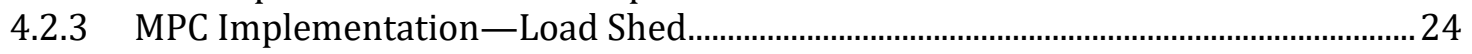

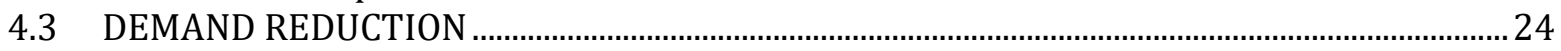

4.3.1 PBC Implementation-Demand Reduction.................................................................25

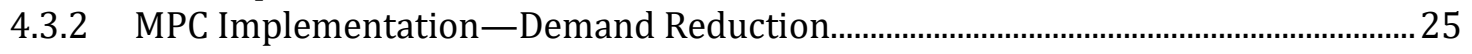

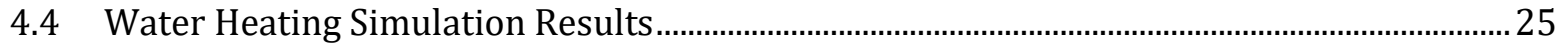

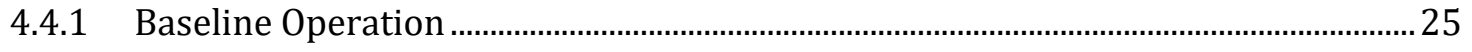

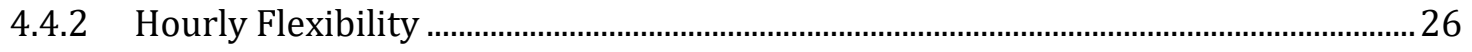

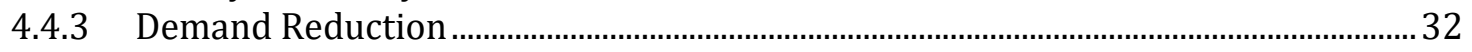

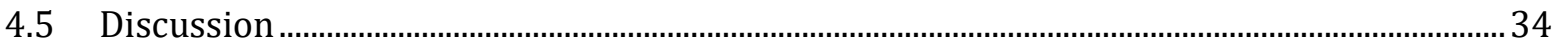

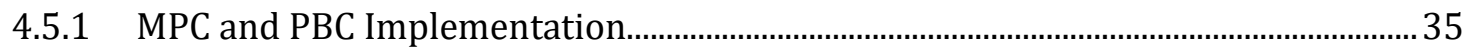

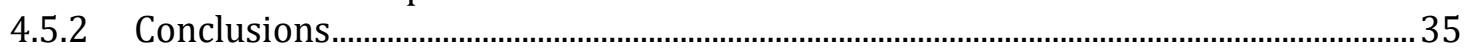

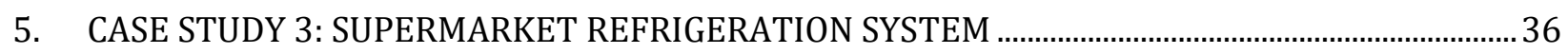

$5.1 \quad \mathrm{CO}_{2}$ open-case refrigerator display case thermal model ........................................................... 36

5.1.1 Simplified Compressor Model for Medium Temperature Refrigeration Cycle........38

5.1.2 Training and Testing Results of the Developed Models.................................................39

5.1.3 Hourly Load Flexibility Through Priority-Based Control............................................... 44

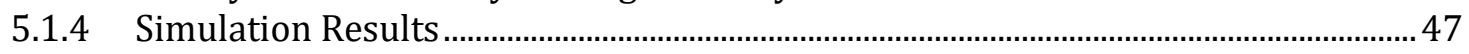

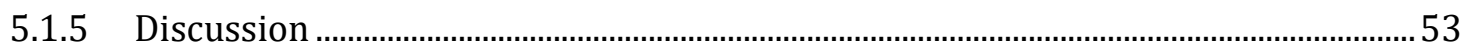




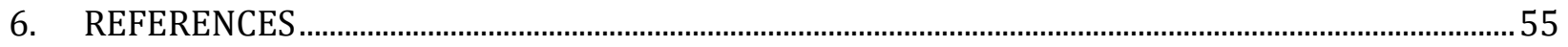




\section{EXECUTIVE SUMMARY}

The emergence of connected devices and the Internet of Things has opened the door to the concept of widespread use of building equipment for load-side flexibility. This stems from the increased use of distributed and clean energy resources for electric generation and the growing demand for a more resilient and reliable grid [1].

A significant challenge associated with the use of building equipment as a distributed energy resource is quantifying the magnitude of power or energy flexibility. Unlike batteries that have well-defined and relatively constant capacity and maximum charge/discharge rates, building equipment often has characteristics that vary based on external factors (e.g., weather or occupant behavior). This makes quantifying the availability and flexibility of these resources difficult.

Controlling a fleet of equipment while minimizing any adverse impacts to the occupants (e.g., discomfort or food spoilage) is a nontrivial matter. Although there are many different control methodologies available, this study focuses on two: priority-based control (PBC) and modelpredictive control (MPC). These were selected because they represent two ends of the control spectrum. PBC is a relatively simple control strategy that requires only the current state of the devices to be controlled and an estimate of how much power each device consumes when turned on. MPC is much more complex and requires a model of the system and forecasts of any disturbances that influence the system. Because MPC has a predictive element, it can prepare for a desired response before the event and therefore is expected to provide a more optimal control relative to PBC. Both MPC and PBC are compared with a baseline simulation that uses feedback control typical of the thermostatically controlled devices being evaluated.

This report investigates the load flexibility of three building systems: residential air conditioners, residential water heaters, and commercial refrigeration. Air heating and cooling and water heating account for $45 \%$ of residential electricity use [2], making them good candidates for providing load flexibility. Refrigeration in commercial buildings accounts for $16 \%$ of commercial building electricity use [3], making it another good candidate.

Simplified models of each system were generated and validated based on measured data from actual equipment. These models were used to simulate the thermal and power response of the systems using PBC and MPC for a fleet of devices trying to achieve a variety of different responses, including hourly load shed, hourly load up, and daily peak demand reduction.

Significant findings from each piece of equipment follow.

\section{RESIDENTIAL AIR CONDITIONERS}

- The averaged cost savings with MPC compared with the baseline control is $10.6 \%$. The savings varies from $3.2 \%$ to $23.3 \%$ with respect to the prediction horizon, comfort bound, and time of use price structure. The savings potential increases with a larger comfort bound, longer prediction horizon, and larger on-peak-to-off-peak price ratios.

- The target-hour demand shedding potential of MPC is $94 \%$ to $99.8 \%$ relative to the baseline (peak power reduction of up to $2 \mathrm{~kW}$ per air conditioner and energy reduction of up to $1.6 \mathrm{kWh}$ per $\mathrm{AC}$ ) while that of $\mathrm{PBC}$ is about $40 \%$ compared with the baseline.

- In the target-hour load-up simulation, about $85 \mathrm{kWh}(1.7 \mathrm{kWh}$ per $\mathrm{AC})$ of additional electricity can be consumed per hour relative to the baseline. 
- The demand shedding potential of PBC ranges from $18.2 \%$ to $31.1 \%$ in each city compared with the baseline case. The potential of MPC ranges from $24.7 \%$ to $41.8 \%$ and from $25.6 \%$ to $46.0 \%$ in $3{ }^{\circ} \mathrm{C}$ and $4^{\circ} \mathrm{C}$ comfort bounds. For the Knoxville weather case, the demand reduction potential for MPC is around $40 \%$ or $800 \mathrm{~W}$ per $\mathrm{AC}$.

- The monthly total cost reduction percentage with the demand shedding strategy is $2.4 \%$ to $11.3 \%$ and $4.0 \%$ to $13.2 \%$ in $3^{\circ} \mathrm{C}$ and $4^{\circ} \mathrm{C}$ comfort bounds with different demand costs.

\section{RESIDENTIAL WATER HEATERS}

- The hourly power reduction potential follows a pattern that closely matches that of hot water use. This results in the highest load reduction potential during weekdays from 6:00 am to 9:00 am. The peak power reduction is $\sim 1,000 \mathrm{~W}$ per water heater, and the energy reduction is $\sim 800 \mathrm{Wh}$ per water heater.

- The hourly power increase potential is highest during the night and early morning when there is little hot water use, but there is still an opportunity for the water heaters to be turned on to recover from tank losses. The maximum sustained power increase over an hour is $\sim 500 \mathrm{~W}$ per water heater for midnight to 4:00 am. The energy use increase over the hour for this time frame is $\sim 500 \mathrm{Wh}$ per water heater. During other hours of the day this falls to $250 \mathrm{Wh}$ per water heater. The power increase potential is similar for weekdays and weekends.

- The daily demand reduction potential for water heaters is $\sim 900$ to $1,200 \mathrm{~W}$ per water heater for weekdays and 600 to $700 \mathrm{~W}$ per water heater for weekends.

- Both PBC and MPC performed very well when controlling a fleet of water heaters to achieve a desired power change. It is likely that simple control methodologies like PBC will be easier to implement and provide similar results to more advanced algorithms.

\section{COMMERCIAL REFRIGERATION}

- The power reduction potential of refrigerators is related to ambient temperature, initial statuses (e.g., defrost status, recover status, air-in-case temperature, food temperature, case temperature, and accumulated running time), and parameter values $\left(C_{f o o d}\right)$. There is no significant power peak for the baseline simulation with variations due to case defrost patterns having a similar magnitude because of changes in outdoor air temperature. If the outdoor air temperature had larger swings between day and night, this might not be the case.

- Typical peak power reduction over the course of an hour does not vary significantly with time of day and is more closely tied to the defrost pattern of the cases. This value is around $300 \mathrm{~W}$ per refrigerated case. The energy reduction potential for each hour is $\sim 250 \mathrm{Wh}$ per refrigerated case.

- Peak power increase potential over an hour is not significant and energy increase potential for an hour is also small, averaging less than $50 \mathrm{Wh}$ per case.

- Note that the results of this study look only at one control mechanism, turning refrigeration on or off to the display cases. Given the complexity of refrigeration systems, there are many other control mechanisms that could yield increased power flexibility. These options are being investigated and include changing case set point temperatures, changing the compressor target suction saturation temperature, directly controlling the variable speed compressor, directly controlling the fixed speed compressors, and adjusting and coordinating the defrost schedules of the cases. 


\section{INTRODUCTION}

The emergence of connected devices and the Internet of Things have opened the door to the concept of widespread use of building equipment for load-side flexibility. This stems from the increased use of distributed and clean energy resources for electric generation, and the growing demand for a more resilient and reliable grid [1].

A significant challenge associated with the use of building equipment as a distributed energy resource is quantifying the magnitude of power or energy flexibility. Unlike batteries that have well-defined and relatively constant capacity and maximum charge/discharge rates, building equipment often has characteristics that vary based on external factors (e.g., weather or occupant behavior). This makes quantifying the availability and flexibility of these resources difficult.

To address this challenge, this study takes several approaches to characterizing the flexibility of different building equipment to provide results that are representative of typical operation of a fleet of units. The end result is a quantification of the average equipment flexibility on a per unit basis that could be expected when controlling a fleet of units.

Residential buildings consume 4.3 quads of electricity annually, approximately $37 \%$ of the total US electricity consumption [2]. Heating, ventilation, and air-conditioning (HVAC) and water heating account for $32 \%$ and $14 \%$ of residential electricity use, respectively, making them prime candidates for demandside load control [2]. Commercial buildings consume 4.2 quads of electricity annually, with refrigeration accounting for $16 \%$ of this. These three building resources are evaluated for their flexibility in this study.

\section{METHODOLOGY}

The flexibility of the equipment is analyzed through the use of simulations. This requires models of the equipment and its associated thermal storage (the house for HVAC, water storage tank for water heating, and display case for commercial refrigeration). The general approach for the models is described in Section 2.1, while the detailed model information for each piece of building equipment is located in its respective section: 3,4 , or 5 .

Controlling a fleet of equipment while minimizing any adverse impacts to the occupants (e.g., discomfort or food spoilage) is a nontrivial matter. Although there are many different control methodologies available, this study focuses on two: priority-based control (PBC) and model-predictive control (MPC). These were selected because they represent two ends of the control spectrum. PBC is a relatively simple control strategy that requires only the current state of the devices to be controlled. MPC is much more complex and requires a model of the system and forecasts of any disturbances that influence the system. Because MPC has a predictive element, it can prepare for a desired response before the event and therefore is expected to provide more optimal control relative to PBC. Both MPC and PBC are compared with a baseline simulation that uses feedback control typical of the thermostatically controlled devices being evaluated.

\subsection{MODELING APPROACH}

\subsubsection{State-Space Formulation}

Thermal systems of buildings are built from heat balance equations in the form of first-order differential equations with parameters such as resistance $(\mathrm{R})$ and capacitance $(\mathrm{C})$ and some coefficient multiplied to 
the control input and disturbances. To use the advance control strategies, those systems of equations are formulated with one system of matrix, which is the state-space formulation.

A continuous-time state-space linear system is defined by Equations 1 and 2:

$$
\begin{aligned}
& \dot{x}(t)=A(t) x(t)+B(t) u(t) . \\
& y(t)=C(t) x(t)+D(t) u(u) .
\end{aligned}
$$

These formulations are discretized, which can be implemented on an actual thermal system with measurement and actuation for the system operation/control.

\subsection{CONTROL METHOD}

In this project, we cover a wide range of control methods from conventional feedback (as a baseline) to PBC and MPC. Feedback control and PBC are simple control methods based only on current measured values and set points. While MPC is more advanced and uses equipment models and forecasted inputs (e.g., weather or occupant behavior patterns).

\subsubsection{Feedback Control}

Feedback control is used for baseline simulations of the equipment and is formulated to mimic the behavior of a thermostat. A thermostat is used to maintain a measured temperature within a certain range. To prevent excessive cycling of the equipment, it is typical for a thermostat to have hysteresis between the on/off control and the temperature change. For example, a thermostat might turn on the air conditioner in a home when the temperature increases to $75^{\circ} \mathrm{F}$ but might not turn off the air conditioner until the temperature reaches $73^{\circ} \mathrm{F}$.

\subsubsection{Priority-Based Control}

PBC is a strategy that involves ranking a group of equipment to create a prioritized list of which equipment will be turned on/off first. At each time interval, the current status of all equipment is received and a list is created based on a defined ranking metric. For example, if the desire is to shed load, then a fleet of water heaters may be ordered based on which water heaters are the warmest and currently turned on. In this way the water heaters that are the hottest are shut off first limiting the amount of discomfort that could be experienced by the occupants. Since PBC considers only the current status of all equipment, power and energy use can only be deferred to a later time.

\subsubsection{Model-Predictive Control}

MPC is a strategy that uses models and forecasts to predict operation of the equipment. Constraining equations and an objective function are then used to achieve optimal performance. Constraints may be used to limit the temperature range in which the equipment is operated to ensure comfort. The objective function could then be defined as the aggregate power or energy use of a fleet over a desired time range that would be minimized during the optimization process. Since MPC uses a model and forecasting, it is capable of shifting energy use both earlier and later in time. 


\section{CASE STUDY 1: RESIDENTIAL AC}

\subsection{RESIDENTIAL MODELS}

\subsubsection{Building Model}

The models were tested by training the $\mathrm{R}$ and $\mathrm{C}$ values of the model using a particle swarm optimization (PSO) routine in $\mathrm{R}$ software. Once the optimal $\mathrm{R}$ and $\mathrm{C}$ values were determined through training, the values were validated by forecasting the indoor temperature of a different set of data. After several iterations of increasing the model complexity, the 4R4C model was found to provide good results during validation and is explained in detail subsequently and in Reference [4].

Figure 1 shows a schematic of the 4R4C gray-box, simplified building model that was developed. Building physical properties that affect thermal transfer are mainly those of exterior walls, roof, and internal mass, which are handled separately in this model. The developed model can therefore reflect the thermal status and response of different building components. Note that only sensible load is considered in this model. Meanwhile, all Rs and Cs are assumed to be time invariant. This model is able to capture the thermal $\mathrm{C}$ of the exterior walls, indoor air, interior walls and furnishings, as well as the attic. All of these items can have a significant effect on the temperature response of the indoor air.

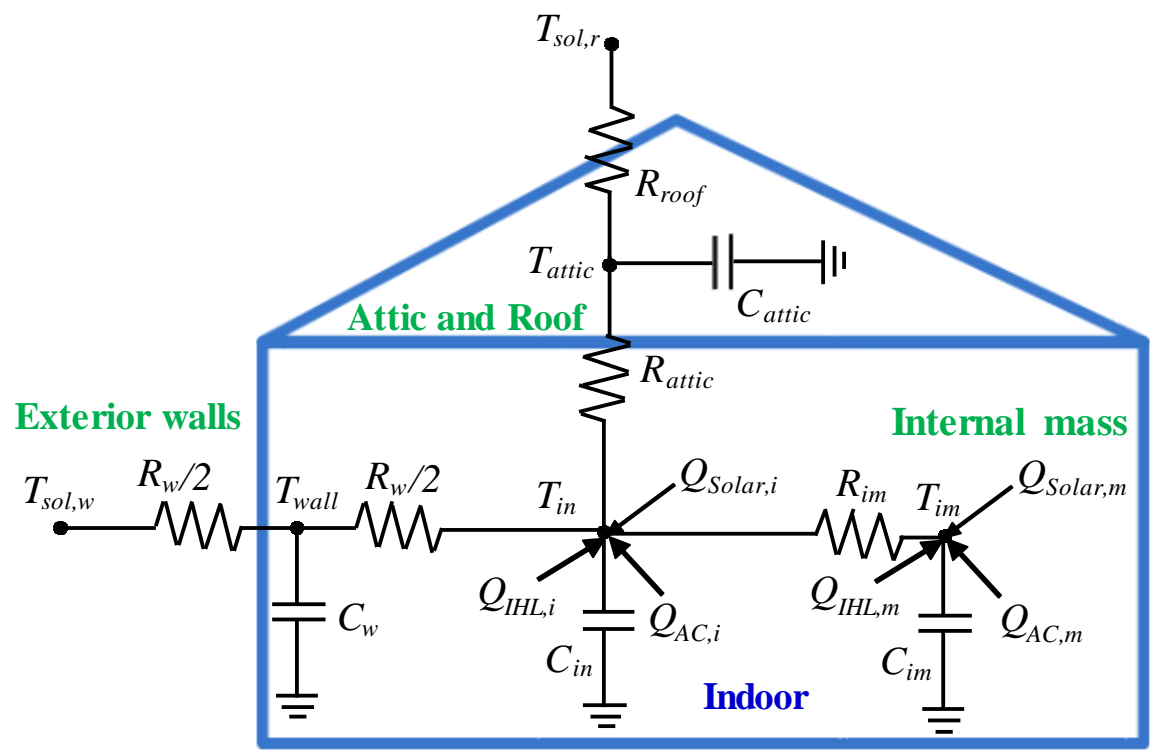

Figure 1: Schematic of the simplified building thermal network model (4R4C).

The heat transfer in the building model is described using Equations 3, 4, 5, and 6.

$$
\begin{gathered}
C_{w} \frac{d T_{\text {wall }}(t)}{d t}=\frac{T_{\text {sol, },}(t)-T_{\text {wall }}(t)}{R_{w} / 2}-\frac{T_{\text {wall }}(t)-T_{\text {in }}(t)}{R_{w} / 2} . \\
C_{\text {in }} \frac{d T_{\text {in }}(t)}{d t}=\frac{T_{\text {wall }}(t)-T_{\text {in }}(t)}{R_{w} / 2}+\frac{T_{\text {attic }}(t)-T_{\text {in }}(t)}{R_{\text {attic }}}+\frac{T_{\text {im }}(t)-T_{\text {in }}(t)}{R_{\text {im }}} \\
+C_{1} s p_{1} Q_{I H L, i}+C_{2} s p_{2} Q_{A C, i}+C_{3} s p_{3} Q_{\text {solar }, i}
\end{gathered}
$$




$$
\begin{gathered}
C_{\text {attic }} \frac{d T_{\text {attic }}(t)}{d t}=\frac{T_{\text {sol, } r}(t)-T_{\text {attic }}(t)}{R_{\text {roof }}}-\frac{T_{\text {attic }}(t)-T_{\text {in }}(t)}{R_{\text {attic }}} . \\
C_{i m} \frac{d T_{i m}(t)}{d t}=-\frac{T_{i m}(t)-T_{\text {in }}(t)}{R_{i m}}+C_{1}\left(1-s p_{1}\right) Q_{I H L, m}+C_{2}\left(1-s p_{2}\right) Q_{A C, m}+C_{3}\left(1-s p_{3}\right) Q_{\text {solar }, m}
\end{gathered}
$$

$C_{w}, C_{a t t i c}, C_{i m}$, and $C_{i n}$ are the thermal capacitances of exterior wall, air in attic, internal mass, and indoor air, respectively. $R_{w}, R_{\text {attic }}, R_{r o o f}$, and $R_{i m}$ are the thermal resistances of exterior walls, attic floor, roof, and internal mass, respectively. $T_{\text {in }}$ is the (average) (equivalent) indoor temperature. $T_{\text {wall }}$ is the (equivalent) envelope temperature. $T_{i m}$ is the (equivalent) indoor thermal mass. $T_{a t t i c}$ is the attic temperature. $T_{s o l, w}$ and $T_{s o l, r}$ are the sol-air temperatures on walls and roof, respectively; they represent the overall effects of outdoor ambient temperature and solar radiation on walls/roof. $Q_{\text {solar }}(\mathrm{W})$ is solar radiation through windows.

One assumption in this research is that the available information is limited in terms of numbers of measure points (e.g., only one indoor temperature data measurement is available) and specific properties of building materials. The effective heating/cooling gain coefficients $C_{1}, C_{2}$, and $C_{3}$ are therefore introduced as one main innovation. $C_{1}, C_{2}$, and $C_{3}$ are used to adjust $Q_{I H L}, Q_{A C}$, and $Q_{\text {solar }}$ for unknown factors. All $C_{1}, C_{2}$, and $C_{3}$ data are assumed to be unknown and need to be identified by the searching algorithm. $S p_{1}, S p_{2}$, and $S p_{3}$ are the convection fractions, which are also identified.

Building internal mass includes floors, interior partitions, furniture, etc. It absorbs radiant heat through the windows and from occupants, lighting, etc. It then releases (or absorbs) heat gradually to the air via convection [5]. Because the effect of building internal mass on cooling/heating energy consumption and indoor temperature is significant, it is necessary to independently consider the building internal mass.

$Q_{I H L}$ is the sensible heat gains from indoor heat resources (W) (e.g., human, equipment, and lighting) and is estimated by Equation 7, which takes the total electricity use of the house and subtracts the electricity sources that do not convert their electricity use to heat within the building envelope. $Q_{A C}$ is the total cooling capacity $(\mathrm{W})$ of the AC.

$$
Q_{I H L}=W_{\text {house }}-W_{A C}-W_{W H}-W_{\text {dryer }}-W_{\text {garage }}-W_{\text {ex }, l} .
$$

The searching process for optimal values of the undetermined parameters in this model is a nonlinear optimization process. Given a set of parameters, the gray-box model can predict the indoor air temperature $\left(T_{i n}\right)$ profile. An objective function is used to evaluate the fitness between the predicted results and measured data collected from the reference building during the training period. The objective function $J$ of such optimization is to minimize the integrated root-mean-square error (RMSE), as defined in Equation 8:

$$
J\left(R_{w}, R_{\text {roof }}, R_{\text {attic }}, R_{\text {im }}, C_{w}, C_{i m}, C_{\text {attic }}, C_{i n,}, C_{1}, C_{2}, C_{3}\right)=\sqrt{\frac{\sum_{k=1}^{N}\left(T_{i n, a c t}-T_{i n, s i m u}\right)^{2}}{N-1}}
$$

where, $T_{i n, a c t}$ is the measured building indoor dry bulb temperature and $T_{i n, s i m u}$ is the indoor temperature resulting from the model. The parameters are identified by a PSO method. PSO is a computational method that optimizes a problem by iteratively trying to improve a candidate solution with regard to a given measure of quality. It solves a problem by having a population of candidate solutions and moving these particles around in the search-space according to simple mathematical formulae over the particle's position and velocity.

The data collected from the reference building in different consecutive time periods with various operating conditions (e.g., different schedules of air conditioner [AC] indoor temperature set points, as 
well as different outdoor weather conditions) are used to train and validate the model. The data collected from September 1-26, 2012, are used for the training session (Figure 2). The indoor temperature set point is scheduled as $20^{\circ} \mathrm{C}$ from 9:00 a.m. to 1:00 p.m., $\sim 27.8^{\circ} \mathrm{C}$ from 1:00 a.m. to 7:00 p.m., and $\sim 25.6^{\circ} \mathrm{C}$ from 7:00 p.m. to 9:00 a.m. the next day. $T_{i n, a c t}$ is the measured indoor temperature, and $T_{i n, s i m u}$ is the predicted indoor temperature from the model. Table 1 lists the resulting parameters identified by PSO.

Table 1: Building model parameters identified by PSO training

\begin{tabular}{cc}
\hline Variable & Value \\
\hline$R_{w}$ & $8.52 \times 10^{-3} \mathrm{~K} / \mathrm{W}$ \\
$R_{\text {attic }}$ & $3.44 \times 10^{-2} \mathrm{~K} / \mathrm{W}$ \\
$R_{\text {roof }}$ & $3.11 \times 10^{-4} \mathrm{~K} / \mathrm{W}$ \\
$R_{\text {im }}$ & $6.61 \times 10^{-3} \mathrm{~K} / \mathrm{W}$ \\
$C_{w}$ & $9.72 \times 10^{6} \mathrm{~J} / \mathrm{K}$ \\
$C_{\text {attic }}$ & $5.02 \times 10^{5} \mathrm{~J} / \mathrm{K}$ \\
$C_{\text {im }}$ & $2.00 \times 10^{7} \mathrm{~J} / \mathrm{K}$ \\
$C_{\text {in }}$ & $6.67 \times 10^{6} \mathrm{~J} / \mathrm{K}$ \\
$C_{1}$ & 0.732 \\
$C_{2}$ & 0.495 \\
$C_{3}$ & 0.050 \\
$S p_{1}$ & 0.913 \\
$S p_{2}$ & 0.920 \\
$S p_{3}$ & 0.111 \\
\hline
\end{tabular}

The data collected from July 1-24, 2011, and from Oct 1-20, 2011, are used for validation. The results are shown in Figure 3 and Figure 4. From July 1-7, the indoor temperature set point was set as $25.6^{\circ} \mathrm{C}$. From July 8-24, the set point was scheduled as $29.4^{\circ} \mathrm{C}$ from 12:00 p.m. to 5:00 p.m., $\sim 28.3^{\circ} \mathrm{C}$ from 5:00 p.m. to 7:00 p.m., and $\sim 25.6^{\circ} \mathrm{C}$ from 7:00 p.m. to 12:00 p.m. the next day. For October, the set point was $\sim 24.4^{\circ} \mathrm{C}$. 


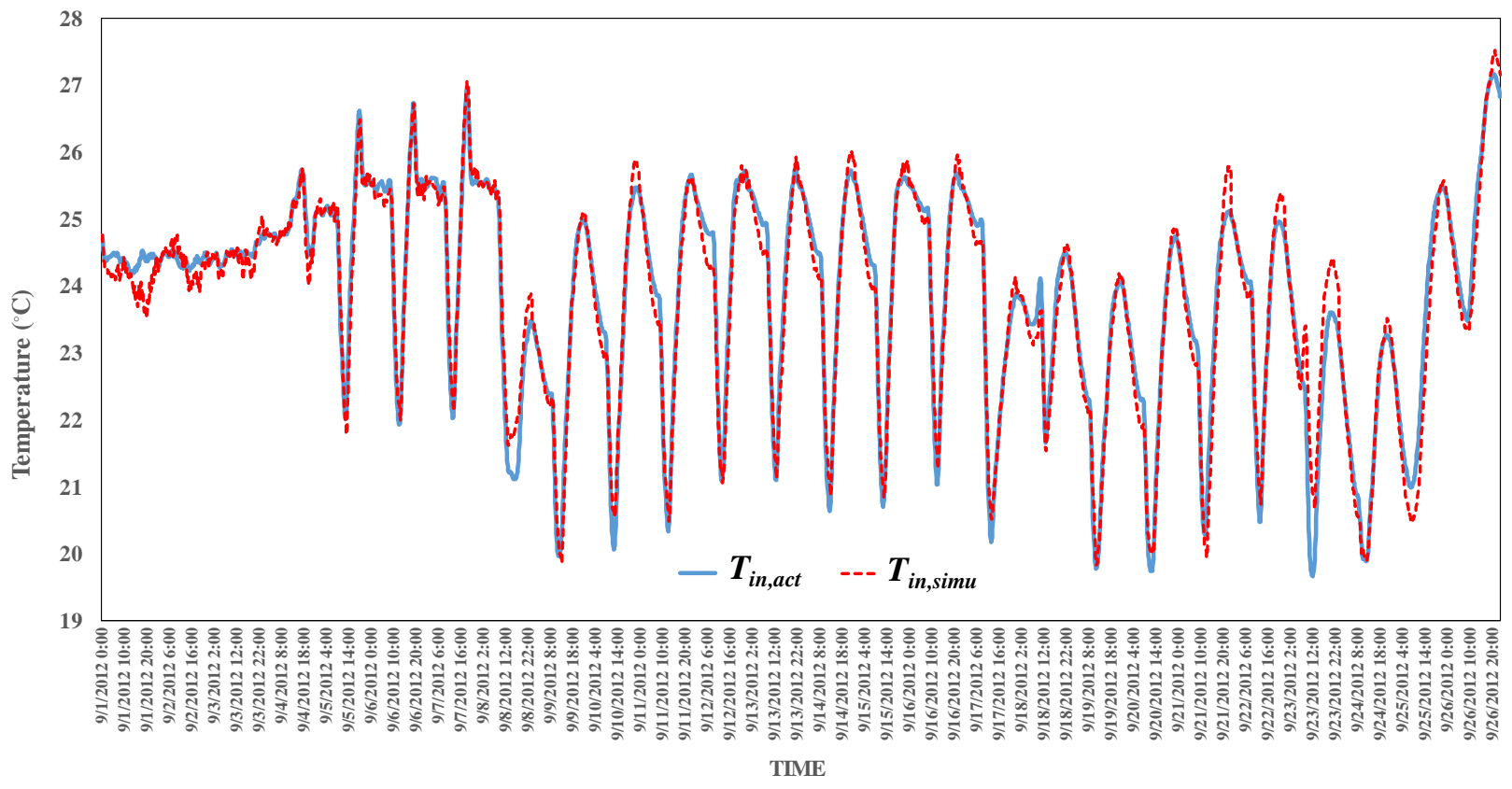

Figure 2: Training results from September 1-26, 2012.

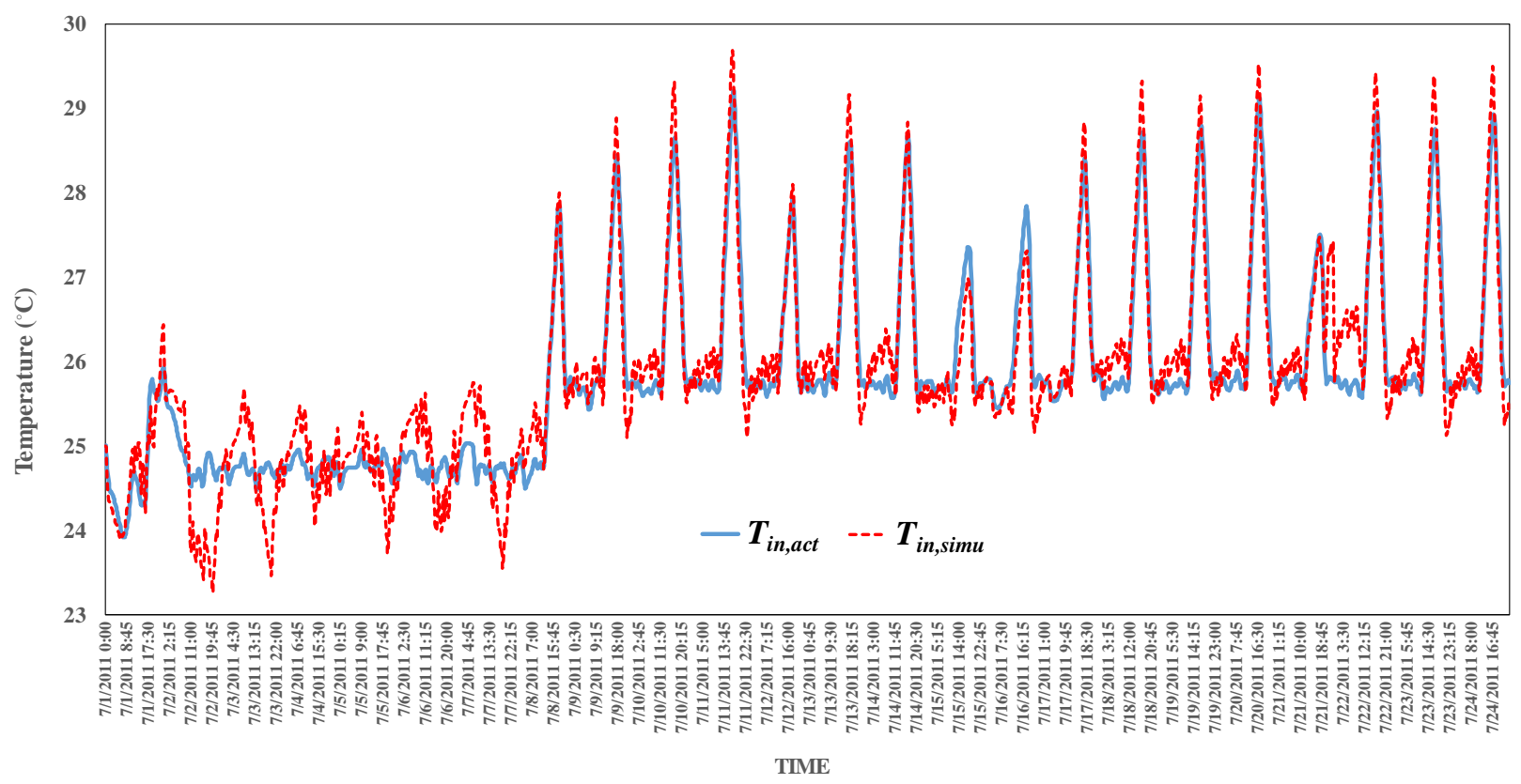

Figure 3: Validation results from July 1-24, 2011. 


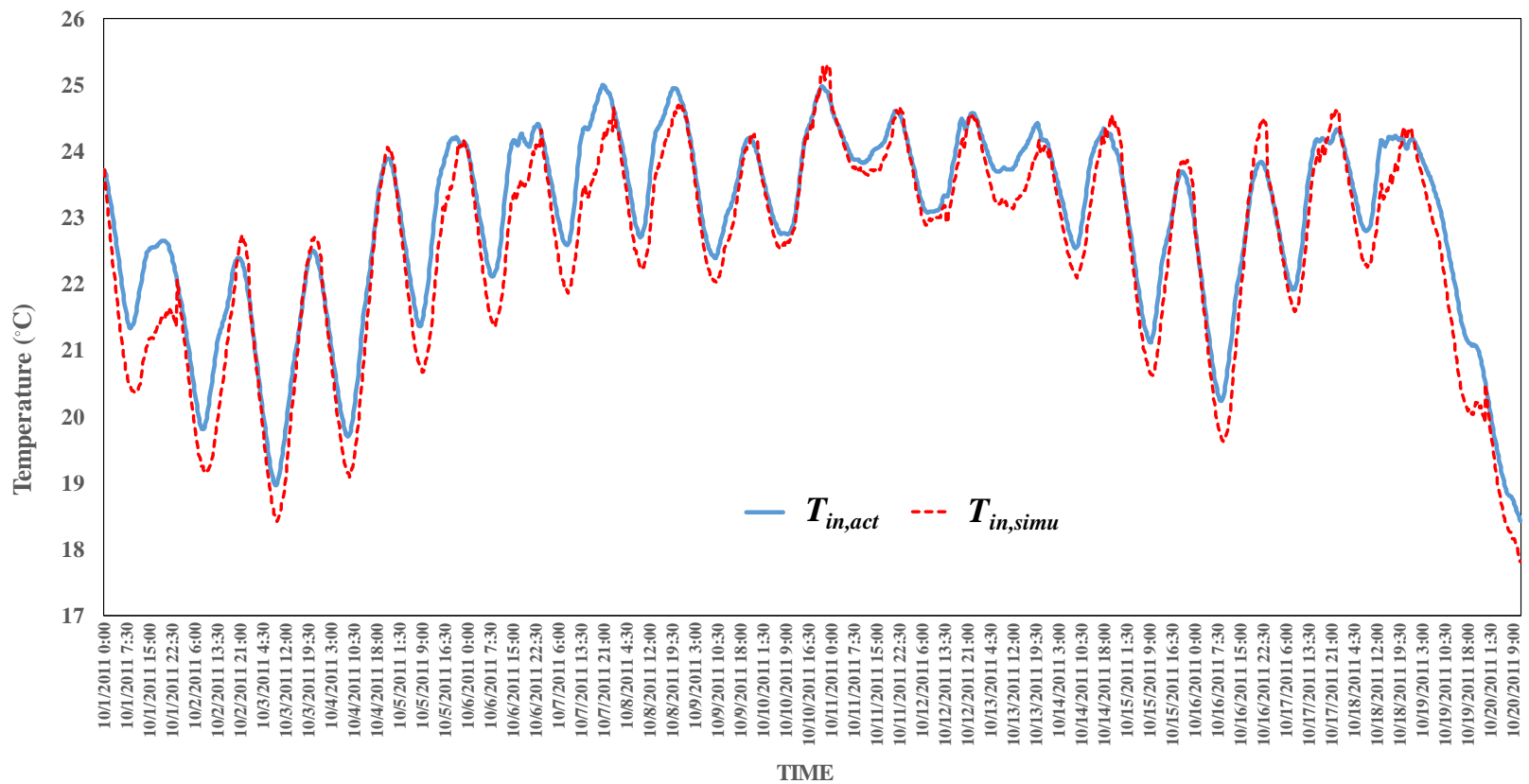

Figure 4: Validation results from October 1-20, 2011.

To quantify the deviations of the predicted data from the measured data in both training session and validation sessions, three indices are used to evaluate the deviations: mean absolute error (MAE), mean absolute percentage error (MAPE), and RMSE.

Table 2 lists the three accuracy indices of the developed model in training and validation sessions. It can be found that the developed model has satisfactory performance in prediction of the building indoor temperature under different scenarios.

Table 2: Accuracy indices of the developed model

\begin{tabular}{ccccc}
\hline Time & Training/validation & $\begin{array}{c}\text { MAE } \\
\left({ }^{\circ} \mathbf{C}\right)\end{array}$ & $\begin{array}{c}\text { MAPE } \\
(\mathbf{\%})\end{array}$ & $\begin{array}{c}\text { RMSE } \\
\left({ }^{\circ} \mathbf{C}\right)\end{array}$ \\
\hline Sep 1-26, 2012 & Training & 0.209 & 0.90 & 0.292 \\
Jul 1-24, 2011 & Validation & 0.300 & 1.16 & 0.388 \\
Oct 1-20, 2011 & Validation & 0.425 & 1.94 & 0.528 \\
\hline
\end{tabular}

\subsubsection{Air Conditioner Model}

The AC used in the simulations is based on a typical, single-stage 13 seasonal energy efficiency ratio unit. The capacity and efficiency are modeled as a function of outdoor temperature using performance curves from BEopt software (Equations 9 and 10) [6]. The curves are also a function of the indoor wet bulb temperature, but for this study a fixed indoor wet bulb temperature of $17.2^{\circ} \mathrm{C}\left(63.0^{\circ} \mathrm{F}\right)$ was assumed. The equations yield multipliers that can be used with the rated capacity and energy input ratio (EIR) to determine the cooling capacity and efficiency at different indoor and outdoor conditions. The rated capacity and EIR are determined at an outdoor dry bulb temperature of $35.0^{\circ} \mathrm{C}\left(95.0^{\circ} \mathrm{F}\right)$ and an indoor wet bulb temperature of $19.4^{\circ} \mathrm{C}\left(67.0^{\circ} \mathrm{F}\right)$. Since the building model considers only the sensible cooling capacity of the AC, it is also assumed that the sensible capacity follows the same trend as the total capacity. 


$$
\begin{gathered}
f_{c a p}=C_{1}+C_{2} T_{i n, w b}+C_{3} T_{i n, w b}^{2}+C_{4} T_{o u t, d b}+C_{5} T_{o u t, d b}^{2}+C_{6} T_{i n, w b} T_{o u t, d b} \\
f_{E I R}=C_{7}+C_{8} T_{i n, w b}+C_{9} T_{i n, w b}^{2}+C_{10} T_{o u t, d b}+C_{11} T_{o u t, d b}^{2}+C_{12} T_{i n, w b} T_{o u t, d b}
\end{gathered}
$$

where:

$T_{i n, w b}=$ indoor wet bulb temperature in degrees Celsius

$T_{\text {out }, d b}=$ outdoor dry bulb temperature in degrees Celsius

$f_{c a p}=$ factor used to correct the rated capacity for different indoor and outdoor conditions

$f_{E I R}=$ factor used to correct the rated EIR for different indoor and outdoor conditions

$C_{1}=1.55736$

$C_{2}=-0.0744482$

$C_{3}=0.00309859$

$C_{4}=0.00145958$

$C_{5}=-4.1148 \times 10^{-5}$

$C_{6}=-0.00042671$

$C_{7}=-0.350448$

$C_{8}=0.116810$

$C_{9}=-0.00339951$

$C_{10}=-0.00122609$

$C_{11}=0.000600809$

$C_{12}=-0.00046688$

\subsection{ANALYSIS APPROACH}

To analyze the load flexibility potential of the homes and ACs, multiple simulations were run using a fleet of 50 homes. The 50 homes were made unique by varying the parameter achieved from the real building training results in Section 3.1.1. The trained values were assumed to be of a typical home, and two additional sets of variables were derived from this set by adjusting the insulation values to improve the envelope. The ranges of the parameters for the three homes are shown in Table 2. Each home efficiency level had randomized small variations in the parameters indicated by the $+/$ - percentages, also shown in Table 2. This adds additional variation to the homes to ensure that homes are not identical. The mix of building efficiency levels in the group of 50 homes is shown in Table 4, with the majority of the homes using parameters that closely match those of the real building used to train the model.

The load flexibility of ACs is analyzed by looking at the overall demand reduction capability, as well as the load shed and load-up flexibility for specific hours of the day. The potential cost savings for the demand reduction case are also investigated for different occupant comfort ranges, time of use rates, demand charges, and climates. 
Table 3: Build parameters for second set of simulations

\begin{tabular}{lccccc}
\hline & Typical & Improved & High Eff & $\begin{array}{c}\text { +Range } \\
(\boldsymbol{\%})\end{array}$ & $\begin{array}{c}\text {-Range } \\
(\boldsymbol{\%})\end{array}$ \\
\hline Rwall & 0.00852 & 0.011797 & 0.0150738 & 5 & 5 \\
Rattic & 0.0344 & 0.043573 & 0.0561867 & 5 & 5 \\
Cwall & 9719515 & 9719515 & 9719515 & 5 & 5 \\
Cin & 6666569 & 6666569 & 6666569 & 10 & 10 \\
C1 & 0.732 & 0.732 & 0.732 & 2 & 2 \\
C2 & 0.495 & 0.495 & 0.495 & 2 & 2 \\
C3 & 0.05 & 0.05 & 0.05 & 50 & 50 \\
Cattic & 501509 & 501509 & 501509 & 10 & 10 \\
Rroof & 0.000311 & 0.000311 & 0.000311 & 5 & 5 \\
Cmass & 19999128 & 19999128 & 19999128 & 25 & 25 \\
Rmass & 0.00661 & 0.00661 & 0.00661 & 5 & 5 \\
Sp1 & 0.913 & 0.913 & 0.913 & 0 & 0 \\
Sp2 & 0.92 & 0.92 & 0.92 & 0 & 0 \\
Sp3 & 0.111 & 0.111 & 0.111 & 0 & 0 \\
Qrated & 4 & 3.6 & 3.2 & 30 & 10 \\
EIRrated & 0.29670435 & 0.284342 & 0.272968 & 5 & 5 \\
EERrated & 11.5 & 12 & 12.5 & & \\
Fraction of load & 1 & 0.9 & 0.8 & & \\
(sizing) & & & & & \\
\hline
\end{tabular}

Table 4: Fraction of each home efficiency level in the group of homes

\begin{tabular}{lc}
\hline Home type & Fraction of total \\
\hline Typical & 0.75 \\
\hline Improved & 0.2 \\
\hline High Eff & 0.05 \\
\hline
\end{tabular}

To investigate the state-wise potential, different weather from a representative nine cities were used. Typical cooling season data were adapted from typical meteorological year data. The data from June, July, and August were averaged respectively, and three days of data were refined as representative summer data for each city, as shown in Figure 5. Those are used to investigate the state-wise impact on shedding potential of MPC in Section 3.4.2.3, whereas the Knoxville-only data where the building model is estimated is used for the rest of simulations. 


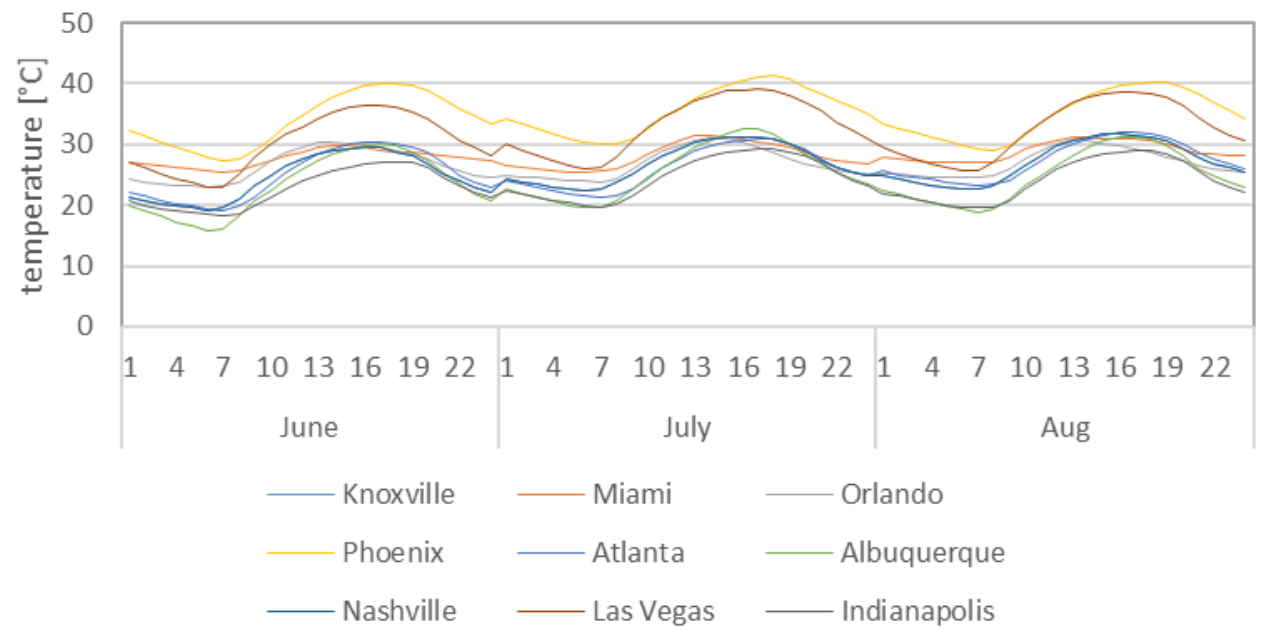

Figure 5: Outdoor air temperatures of nine major cities.

\subsection{CONTROL METHODS}

\subsubsection{Baseline Operation}

For the baseline operation, the thermostat deadband was assumed to be $23.5^{\circ} \mathrm{C}-22.0^{\circ} \mathrm{C}\left(74.3^{\circ} \mathrm{F}-71.6^{\circ} \mathrm{F}\right)$. This means that the $\mathrm{AC}$ was turned on to cool the building when the indoor temperature reached $23.5^{\circ} \mathrm{C}$ or higher and shut off when the indoor temperature reached $22.0^{\circ} \mathrm{C}$ or below. A five-minute time interval was used for control so the AC state is maintained for the entire interval.

\subsubsection{Priority-Based Control}

In the second control strategy, we consider priority-based coordination and control of an aggregate of on/off HVAC systems, which are widely spread in residential buildings in addition to many small-tomedium-size commercial buildings. We show in this section that by proper control and coordination of a fleet of on/off HVAC systems (available in one or many building[s]), this would provide the required flexibility for limiting peak power consumption while limiting the excursion of the indoor temperatures from the desired set points.

Without loss of generality, by considering the cooling case in HVAC units and assuming all buildings have the same temperature set points, the centralized PBC strategy is designed as follows: when shedding load, the controller should shut off units in homes that are closest to the bottom of their deadband, allowing houses that are warmed to continue cooling. So, the controller should decrease the power provided to HVAC units with the lowest temperature zones or buildings.

\subsubsection{Model-Predictive Control}

We developed an MPC strategy for coordinating the operation of many HVAC units to reduce the electricity cost while limiting the peak aggregate power demand and maintaining the comfort of each house in the desired temperature range. The MPC uses simplified building models for each home, along with weather and internal load forecasts and AC performance curves to identify optimal on/off schedules for each system to meet the peak demand reduction.

The estimated parameters in systems of equations (Equations 11 and 12) are constructed with state-space formulation as shown in Equation 2.1 and then discretized with respect to the time step. Input $(u)$ to the model is split to the disturbance input $(w)$ and control input $(U)$ : 


$$
\begin{gathered}
x(t+1)=A_{d} x(t)+B_{d} u(t)=A_{d} x(t)+B_{d, w} w(t)+B_{d, U} U(t), \\
y(t)=C_{d} x(t)+D_{d} u(u),
\end{gathered}
$$

where:

$$
\begin{aligned}
& \mathrm{X}=\left[\mathrm{T}_{\text {wall }} \mathrm{T}_{\text {in }} \mathrm{T}_{\text {attic }} \mathrm{T}_{\text {mass }}\right] \\
& \mathrm{U}=\left[\mathrm{Q}_{\mathrm{AC}}\right] \\
& \mathrm{W}=\left[\mathrm{T}_{\mathrm{sol}, \mathrm{w}} \mathrm{T}_{\mathrm{sol}, \mathrm{r}} \mathrm{Q}_{\mathrm{IHL}} \mathrm{Q}_{\text {solar }}\right]
\end{aligned}
$$

The state-space formulation is stacked in a time series and generates the chunk of matrices. The control input $(U)$ and temperature trajectories $(x)$ are in an explicit linear relation with the observability matrix $\left(\Omega_{\mathrm{x}}\right)$, controllability matrix $\left(\Omega_{\mathrm{w}}\right.$ and $\left.\Omega_{\mathrm{u}}\right)$, and disturbance input $(w)$, as seen in Equation 13 .

$$
\underbrace{\left[\begin{array}{c}
x(1) \\
x(2) \\
\vdots \\
x(n)
\end{array}\right]}_{X}=\underbrace{\left[\begin{array}{c}
A_{d} \\
A_{d}{ }^{2} \\
\vdots \\
A_{d}{ }^{n}
\end{array}\right]}_{\Omega_{x}} \underbrace{x(0)}_{x_{0}}+\underbrace{\left[\begin{array}{cccc}
B_{d, w} & 0 & \cdots & 0 \\
A_{d} B_{d, w} & B_{d, w} & \cdots & 0 \\
\vdots & \vdots & \ddots & \vdots \\
A_{d}{ }^{n-1} B_{d, w} A_{d}{ }^{n-2} B_{d, w} & \cdots & B_{d, w}
\end{array}\right]}_{\boldsymbol{\Omega}_{w}} \underbrace{\left[\begin{array}{c}
w(0) \\
w(1) \\
\vdots \\
w(n-1)
\end{array}\right]}_{w}+\underbrace{\left[\begin{array}{cccc}
B_{d, u} & 0 & \cdots & 0 \\
A_{d} B_{d, u} & B_{d, u} & \cdots & 0 \\
\vdots & \vdots & \ddots & \vdots \\
A_{d}{ }^{n-1} B_{d, u} A_{d}{ }^{n-2} B_{d, u} & \cdots & B_{d, u}
\end{array}\right]}_{\mathbf{\Omega}_{u}} \underbrace{\left[\begin{array}{c}
u(0) \\
u(1) \\
\vdots \\
u(n-1)
\end{array}\right]}_{U} .
$$

Then optimization for one house (referred as $i$ ) is formulated with Equation Error! Reference source not found.:

$$
\begin{gathered}
\min \varepsilon_{\text {cost }} \cdot \underbrace{f_{\text {EIR }, i} \cdot f_{\text {cap }, i}}_{Q_{\text {cap }, i}} \cdot U_{i} \\
\text { such that } \\
U_{i}=0 \text { or } 1, \\
\underbrace{\left[\begin{array}{c}
C_{T} \cdot \Omega_{\mathrm{u}, \mathrm{i}} \cdot Q_{\text {cap }, i} \\
-C_{T} \cdot \Omega_{\mathrm{u}, \mathrm{i}} \cdot Q_{\text {cap }, i}
\end{array}\right]}_{A_{\text {ineq }, i}} U \leq \underbrace{\left[\begin{array}{c}
T_{\text {upper }}-C_{T} \cdot\left(\Omega_{X, i} \cdot \mathrm{X}_{0}-\Omega_{w} \cdot w\right) \\
-T_{\text {lower }}+C_{T} \cdot\left(\Omega_{X, i} \cdot \mathrm{X}_{0}-\Omega_{w} \cdot w\right)
\end{array}\right]}_{B_{\text {ineq }, i}} .
\end{gathered}
$$

The control input is $U_{i}$, which is a vector of control inputs in prediction horizon (i.e., its dimension is identical to the prediction horizon). $C_{T}$ is the predefined matrix for extracting the target temperature from the state (e.g., air temperature). $T_{\text {upper }}$ and $T_{\text {lower }}$ are comfort bounds and are regulated with hard constraint in inequality constraint.

To investigate the demand shedding of aggregated multiple houses, extensive optimization is formulated with additional inequality constraint limiting the summed peak power of all houses lower than the criteria, $Q_{\max }$, as shown in Equation Error! Reference source not found.:

$$
\begin{aligned}
& \min \left[\varepsilon_{\text {cost }} \cdot Q_{c a p, 1} \quad \cdots \quad \varepsilon_{\text {cost }} \cdot Q_{c a p, n}\right] \cdot\left[\begin{array}{c}
U_{1} \\
\vdots \\
U_{n}
\end{array}\right] \\
& \text { such that } \\
& {\left[\begin{array}{c}
U_{1} \\
\vdots \\
U_{n}
\end{array}\right]=0 \text { or } 1}
\end{aligned}
$$




$$
\min \left[\begin{array}{ccc}
A_{\text {ineq }, 1} & 0 & 0 \\
0 & \ddots & 0 \\
0 & 0 & A_{\text {ineq }, n} \\
Q_{\text {cap }, 1} & \cdots & Q_{\text {cap }, n}
\end{array}\right] \cdot\left[\begin{array}{c}
U_{1} \\
\vdots \\
U_{n}
\end{array}\right] \leq\left[\begin{array}{c}
B_{\text {ineq }, 1} \\
\vdots \\
B_{\text {ineq }, n} \\
Q_{\max }
\end{array}\right]
$$

Obviously, this turns out to be a mixed integer programming problem that can be solved by certain commercial solvers, such as Gurobi, CPLEX, and MOSEK.

\subsection{SIMULATION RESULTS}

This section describes the extensive simulation studies carried out to investigate the savings potential of the PBC and MPC in cost and demand charge. A typical electricity price of $\$ 0.127 / \mathrm{kWh}$ is used, which is the state-averaged cost [7]. Also, time of use (TOU) is applied [8]; on-peak price is twice the off-peak price, while their average is $\$ 0.127 / \mathrm{kWh}$.

\subsubsection{MPC Parametric Study}

In the simulation studies, we evaluate the cost-saving potential of the MPC compared with the baseline control (feedback control). The estimated house model is selected for the analysis. Knoxville weather data is used where the building model was estimated. Three different comfort bounds are considered $\left(2^{\circ} \mathrm{C}\right.$, $3^{\circ} \mathrm{C}$, and $4^{\circ} \mathrm{C}$ ). The upper bound, $23.5^{\circ} \mathrm{C}$, is set to the set point for the baseline case. Three different TOU price ratios are used where on-peak price is twice, four times, and six times higher than off-peak price, while the average is still $\$ 0.127 / \mathrm{kWh}$. Five prediction horizons are tested from 2 to 10 hours in 2 hour increments. The parameters for the study are summarized in Table 5.

Table 5: Parameters for MPC study

\begin{tabular}{cccc}
\hline Comfort bound & \multicolumn{2}{c}{ TOU price structure } & $\begin{array}{c}\text { Prediction horizon } \\
(\mathrm{h})\end{array}$ \\
\cline { 2 - 3 } & $\begin{array}{c}\text { Off-peak } \\
(/ \mathrm{kWh})\end{array}$ & $\begin{array}{c}\text { On-peak } \\
(/ \mathrm{kWh})\end{array}$ & \\
\hline $23.5^{\circ} \mathrm{C}-21.5^{\circ} \mathrm{C}\left(74.3^{\circ} \mathrm{F}-70.7^{\circ} \mathrm{F}\right)$ & $\$ 0.0847$ & $\$ 0.1693$ & 2 \\
$23.5^{\circ} \mathrm{C}-20.5^{\circ} \mathrm{C}\left(74.3^{\circ} \mathrm{F}-68.9^{\circ} \mathrm{F}\right)$ & $\$ 0.0508$ & $\$ 0.2032$ & 4 \\
$23.5^{\circ} \mathrm{C}-19.5^{\circ} \mathrm{C}\left(74.3^{\circ} \mathrm{F}-67.1^{\circ} \mathrm{F}\right)$ & $\$ 0.0363$ & $\$ 0.2177$ & 6 \\
& & & 8 \\
& & & 10 \\
\hline
\end{tabular}

Figure 6 shows example results for the comparison of baseline and MPC with a $3^{\circ} \mathrm{C}$ comfort bound $\left(20.5-23.5^{\circ} \mathrm{C}\right), 6$ hour prediction horizon, and TOU structure level 2. MPC tends to consume the energy when the electricity price is low and the AC efficiency is higher, thus shifting the cooling load from onpeak to off-peak price periods by using precooling. MPC uses more energy, $66.3 \mathrm{kWh}$ compared with $65.20 \mathrm{kWh}$ for the baseline over the three days. However, the energy cost is lower for MPC at \$7.51 compared with $\$ 8.59$ for the baseline, which yields $12.62 \%$ cost savings. MPC uses more energy because of precooling. The house is maintained at a lower average temperature than the baseline, which requires more energy use. This increased energy load is partially offset by the higher AC efficiency during the precooling period but not completely. 


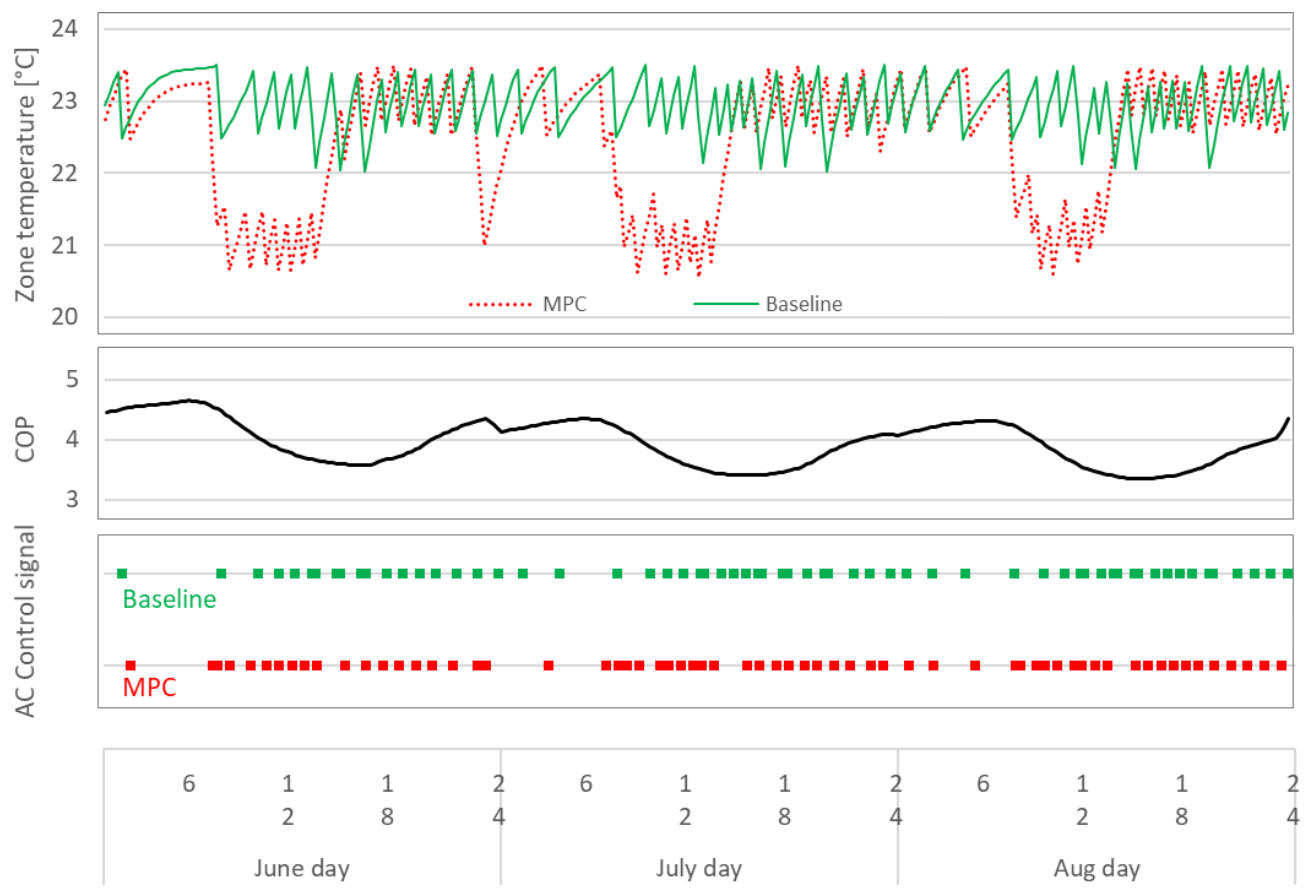

Figure 6: Example of MPC parametric study $\left(3^{\circ} \mathrm{C}\right.$ comfort bound, 6 hour prediction horizon, and TOU structure level 2).

Figure 7 shows the final simulation results of the MPC savings potential compared with the baseline with respect to the TOU price structure, prediction horizon, and comfort range. As expected, the MPC cost savings increases with longer prediction horizon, larger comfort bound, and larger ratio between on-peak and off-peak price. However, a longer prediction horizon (e.g., 10 hours) does not always give good results because of the computational burden of the optimization, which is a nonlinear/nonconvex problem. Higher savings might be achieved with more powerful computation tools or advanced optimization algorithms. Also, the MPC savings potential with narrow comfort bounds (e.g., 2C) is limited even with a higher prediction horizon and large on-peak to off-peak TOU price structures.

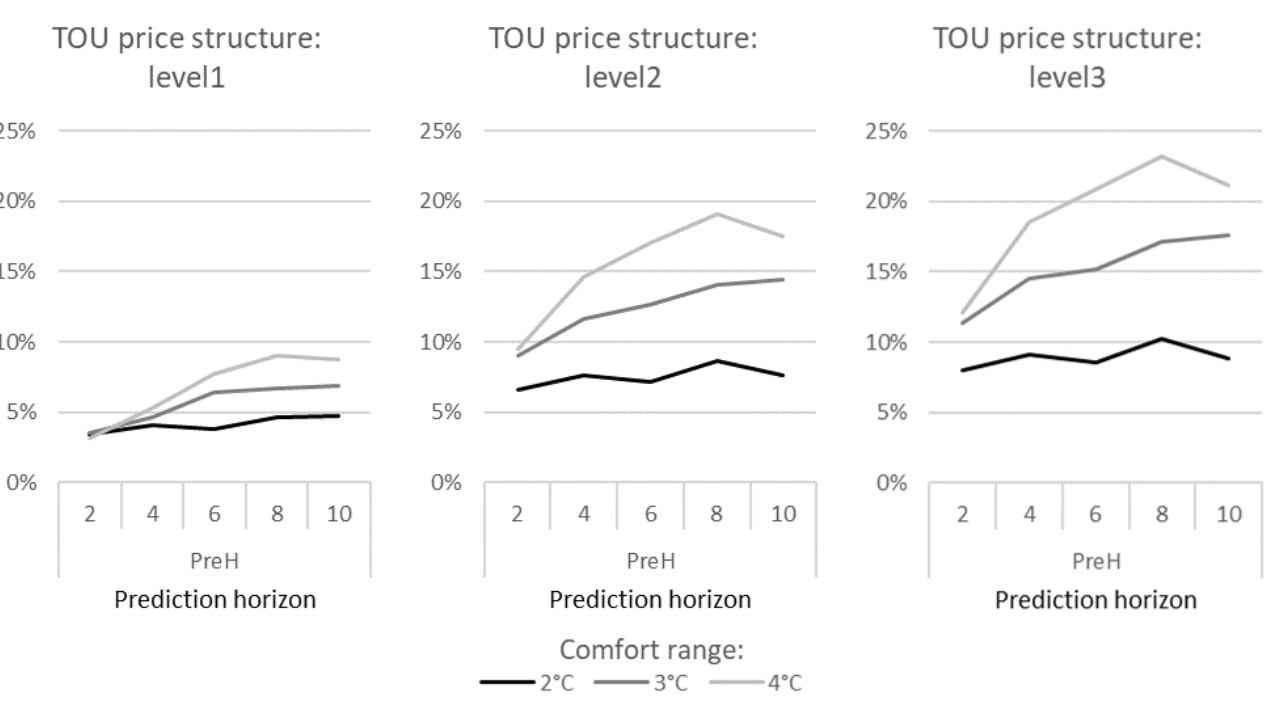

Figure 7: MPC savings potential with respect to TOU price, prediction horizon, and comfort bounds. 


\subsubsection{Hourly Load Flexibility with Demand Charge Analysis}

In this section, load flexibility of residential ACs is simulated with PBC and MPC for 1 hour time intervals. A set of 50 houses using Knoxville weather, as explained in Section 3.23.3.2, are considered. Load shedding and increasing load are independently simulated for each hour of the day for the fleet of 50 homes. Two comfort bounds are considered $\left(2^{\circ} \mathrm{C}\right.$ and $\left.3^{\circ} \mathrm{C}\right)$ for MPC with 4 hours of prediction horizon. The upper bound, $23.5^{\circ} \mathrm{C}$, is set to the set point for the baseline and $\mathrm{PBC}$ (i.e., the room air temperature is not higher than the set point).

Demand shedding potential in a whole simulation period (three days) is evaluated with different weather from major cities in the United States. TOU level 1 (the on-peak price is twice the off-peak price) is used for the electricity price.

\subsubsection{Target-hour shedding}

PBC simulations run with shedding strategy as explained in Section 3.3.2. In each time step, the deviations between the set points and room air temperatures are calculated for all houses, and the most deviated houses (e.g., with the lowest room air temperature) are turned off one by one as long as the room air temperature is not violated (i.e., is not higher than the set point). MPC simulations run by increasing the number of houses simultaneously operating the ACs in each target hour; MPC starts with target hour 1 (midnight to $1 \mathrm{a} . \mathrm{m}$.) turning off all ACs and then increasing the number of ACs (zero to one) if the temperature constraint is violated. This process is repeated for each hour of the day.

Figure 8 shows the shedding with the target hour at 12 p.m. as an example. The maximum number of houses operating the $\mathrm{AC}$ at the target hour of $12 \mathrm{p} . \mathrm{m}$. is 25,14 , and 3 in baseline, PBC, and MPC cases, respectively. The shedding potential of MPC is much higher compared with the $\mathrm{PBC}$ as it is capable of using precooling to shift load earlier in time as well as later (the only capability of PBC). After the shedding, MPC shows a rebound peak. To mitigate the size of this peak, a global constraint is set limiting the number of running units to a maximum of 28 , the maximum number of units running in the baseline case.

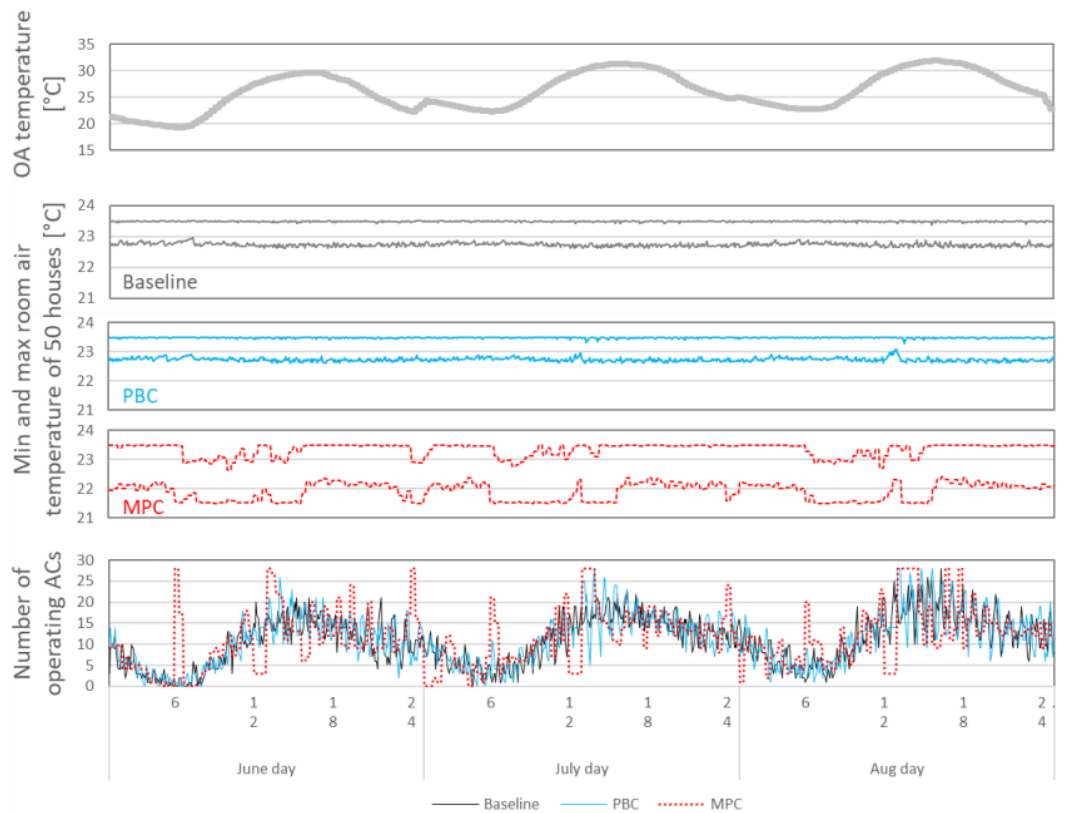

Figure 8: Example of target-hour shedding (12 p.m.-1 p.m.). 
Figure 9 shows the shedding potential of PBC and MPC compared with the baseline for each hour of the day. The results are averaged from three days of simulation (average day of June, July, and August) and the maximum number of houses is picked in each hour. The highest peak of the baseline is 28 , as shown with the black line (left graph). PBC can reduce almost half shedding (40\% on average), while MPC can achieve almost full shedding $\left(94 \%\right.$ and $99.8 \%$ on average with $2{ }^{\circ} \mathrm{C}$ and $3{ }^{\circ} \mathrm{C}$ comfort ranges, respectively).

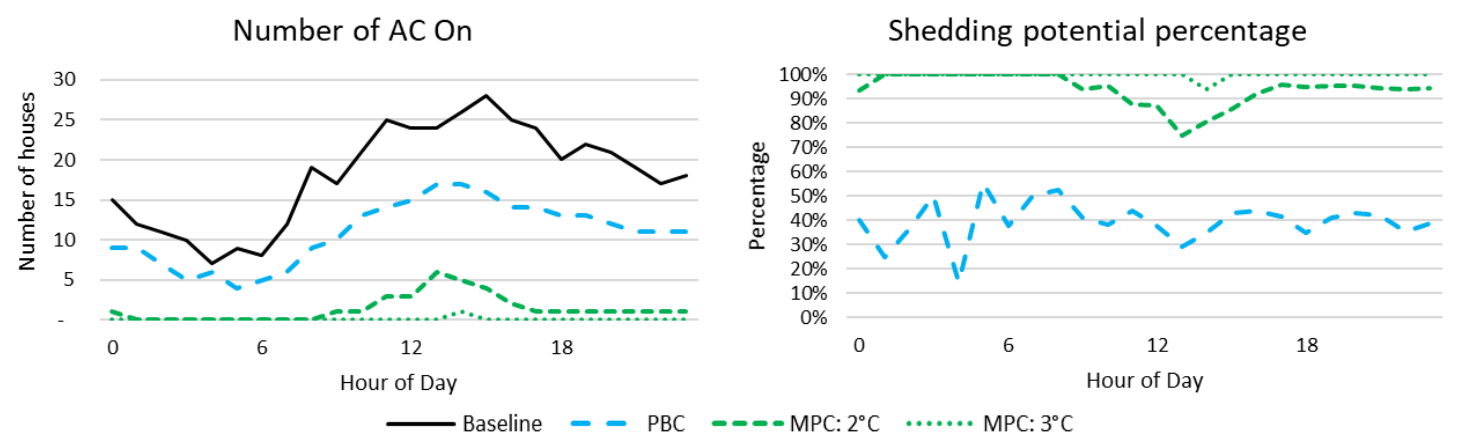

Figure 9: Number of AC on (left) and their shedding percentage (right).

Simulation results in terms of peak and total power are also shown in Figure 10. Shedding potential with peak and total power is decreased in PBC compared with the previous analysis calculating only the number of houses. MPC achieves almost $100 \%$ of the shedding potential of each target hour, for nearly all hours. All 50 ACs can be shut off for an hour with appropriate precooling.

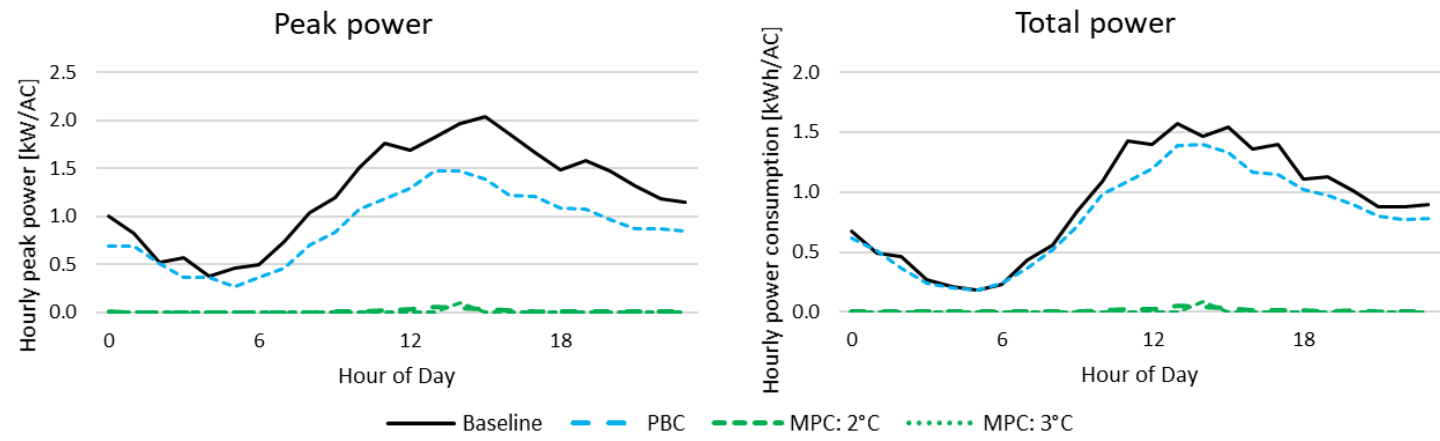

Figure 10: Hourly peak power (left) and total power (right) of one house in shedding case.

\subsubsection{Target-hour load-up}

Considering the case where electricity generated from renewable sources is excessive and it is beneficial to consume power, the maximum power load-up for specific target hours is evaluated. Figure 11 shows the hourly profile of ACs for one normalized house (e.g., divided by 50 houses). Similar with previous shedding cases, room air temperature is maintained in comfort bounds. Only the PBC case is considered, and it is compared with the baseline case. About $1.7 \mathrm{kWh}$ of additional electricity can be used relative to the baseline for each hour with very little variation throughout the day. 


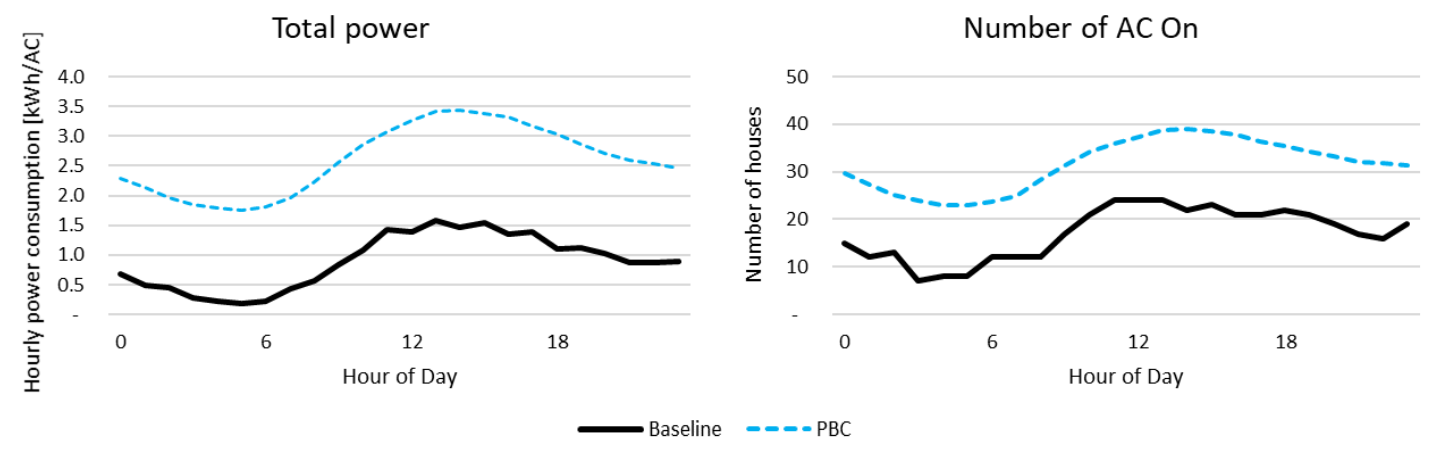

Figure 11: Peak and total power of one house in load-up case.

\subsubsection{24-hour flat shedding}

In this section, demand shedding potential throughout the simulation period is investigated. In these simulations a global power limit is applied based on a percentage of the baseline peak power use $(87.1 \mathrm{~kW})$. This has the effect of flattening the load. Simulations are run until the lowest peak power, across the entire simulation period, is achieved while maintaining the house temperatures within the comfort bounds.

Figure 12 shows the simulations with Knoxville weather. MPC with a $4{ }^{\circ} \mathrm{C}$ comfort bound is able to reduce peak power by $22.5 \%(19.4 \mathrm{~kW})$ compared with $\mathrm{PBC}$ and $46.0 \%(56.7 \mathrm{~kW})$ compared with the baseline case. Shifted cooling load by maximizing the precooling is shown (e.g., AC starts at $\sim 6$ a.m). This savings potential is possible only with the large comfort range of $4{ }^{\circ} \mathrm{C}$, and it decreases to $41.5 \%$ and $16.5 \%$ compared with the baseline case and $\mathrm{PBC}$ case, respectively.

Figure 13 shows the box and whisker plot of outdoor air temperatures in each city, and Figure 14 represents the demand savings potential of $\mathrm{PBC}$ and $\mathrm{MPC}$ with $3^{\circ} \mathrm{C}$ and $4^{\circ} \mathrm{C}$ comfort bounds compared with the baseline. A larger comfort bound increases the demand shedding potential as expected, and a higher outdoor air temperature reduces the demand shedding potential (e.g., Phoenix and Las Vegas). Also, interestingly, the lowest shedding potential is seen in Miami, the minimum air temperature of which is high even though the maximum is not high. 

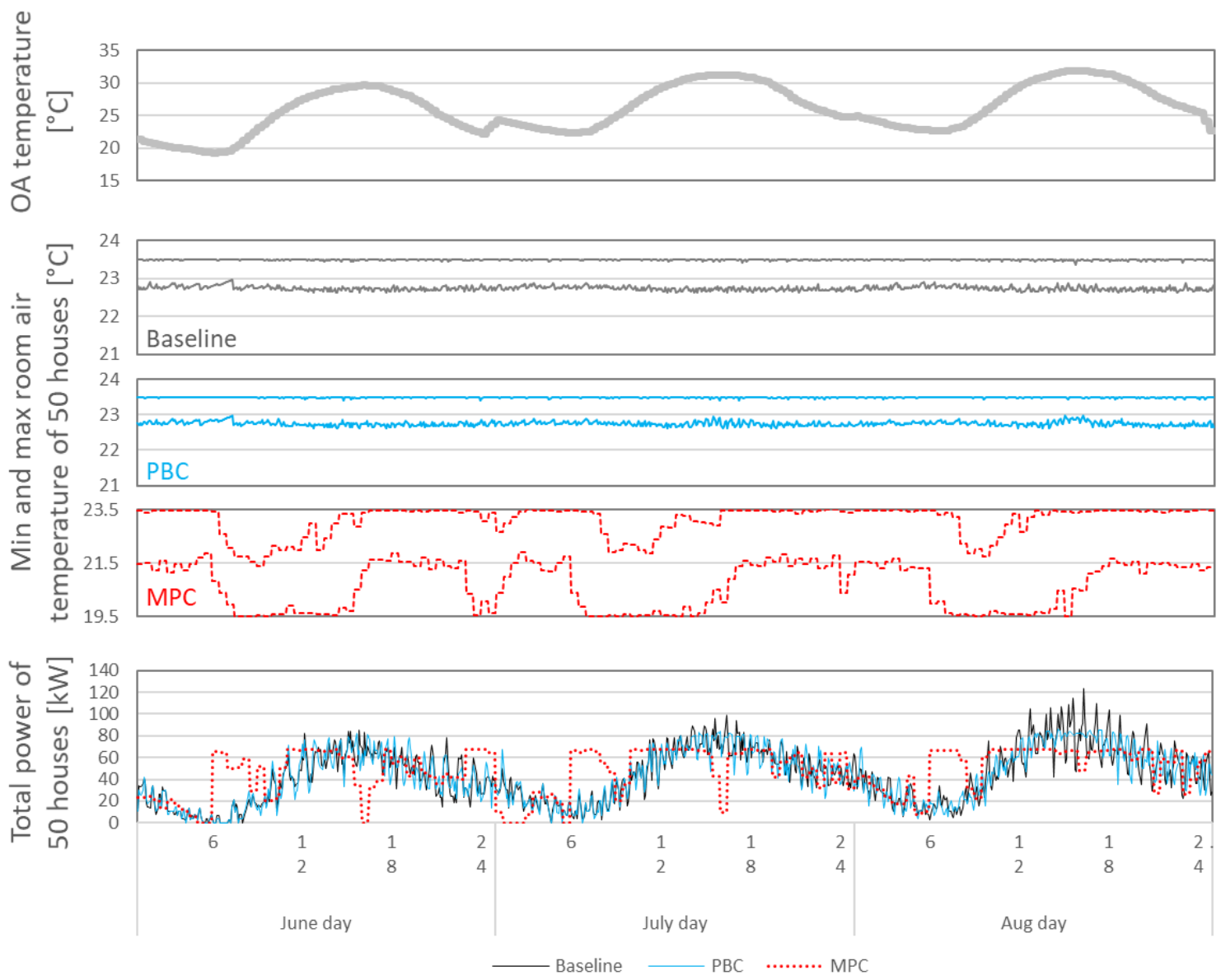

Figure 12: Example of 24 hour shedding (with Knoxville weather).

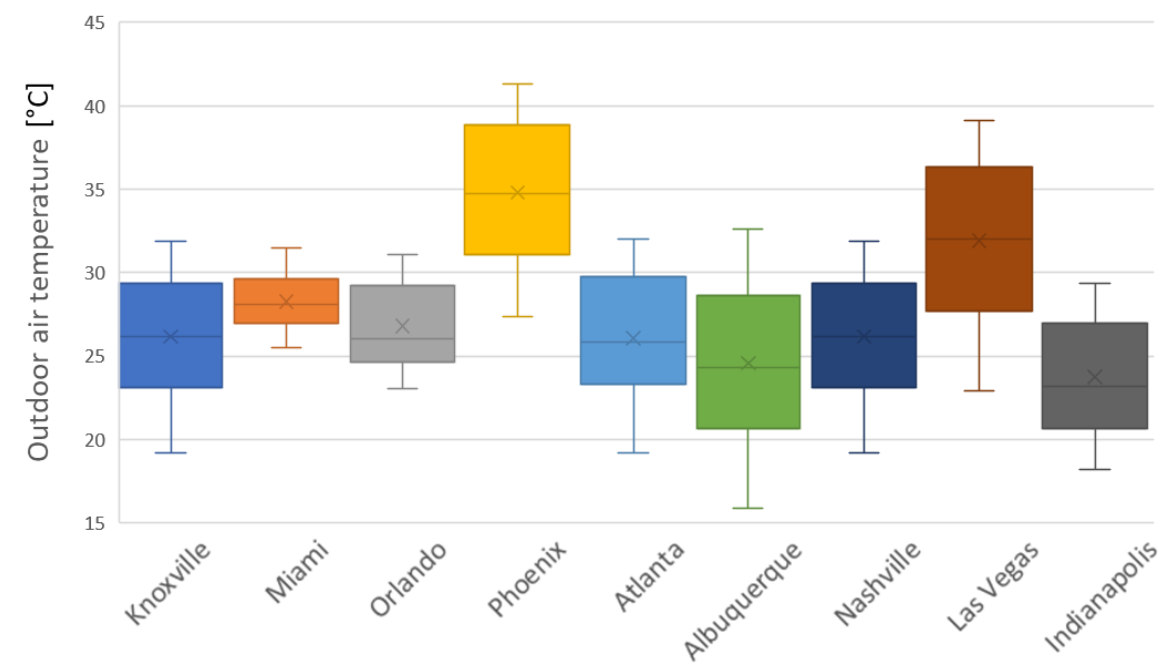

Figure 13: Box and whisker plot of outdoor air temperatures. 


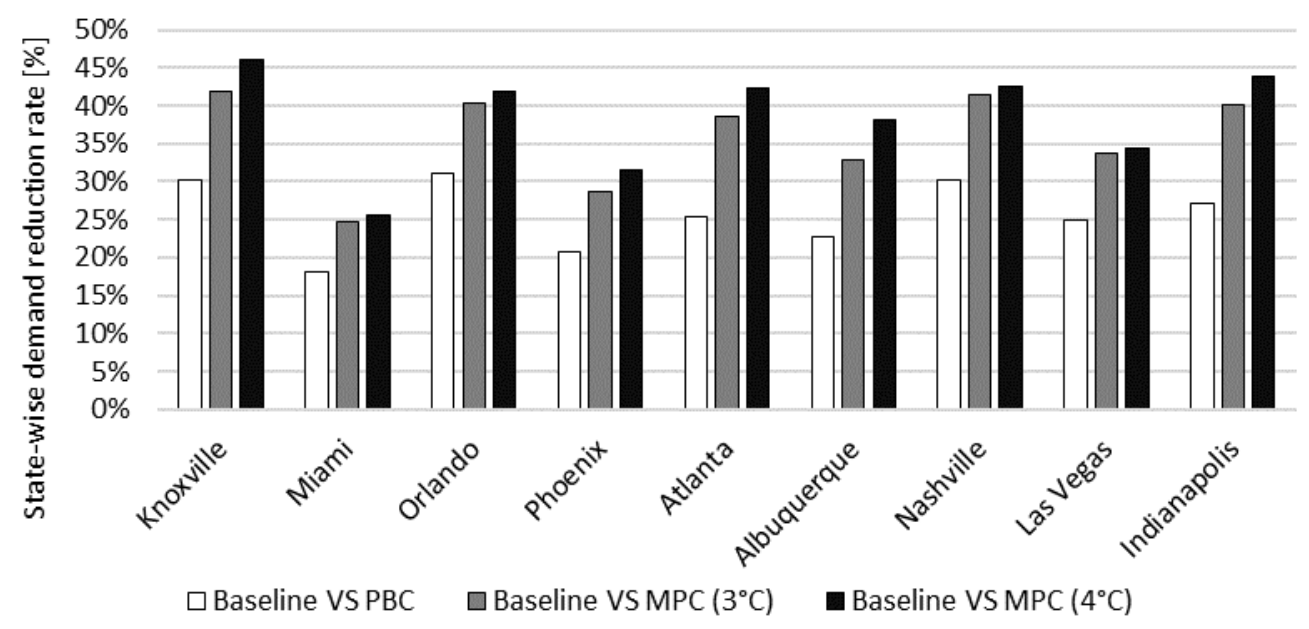

Figure 14: Demand savings potential of each city.

Even with the high savings potential in demand charges due to peak power reduction, overall savings needs to be analyzed in the context of whole energy/electricity usage in a month. Demand charge varies with utilities and states [8], and this study applies four different levels of demand charges: $\$ 5 / \mathrm{kW}$, $\$ 10 / \mathrm{kW}, \$ 15 / \mathrm{kW}$, and $\$ 20 / \mathrm{kW}$. Figure 15 shows the total electricity bill of each city with respect to the different demand cost; the cost is calculated by scaling up the 3 day simulations to 30 days (multiplying by 10). It is observed that the portion of the demand charge becomes large when higher demand cost is introduced. Also, their saving percentages compared with the baseline case are shown in Figure 16. Averaged saving percentages of all cities are $2.4-11.3 \%$ and $4.0-13.2 \%$ for the $3^{\circ} \mathrm{C}$ and $4^{\circ} \mathrm{C}$ comfort bounds, respectively. 


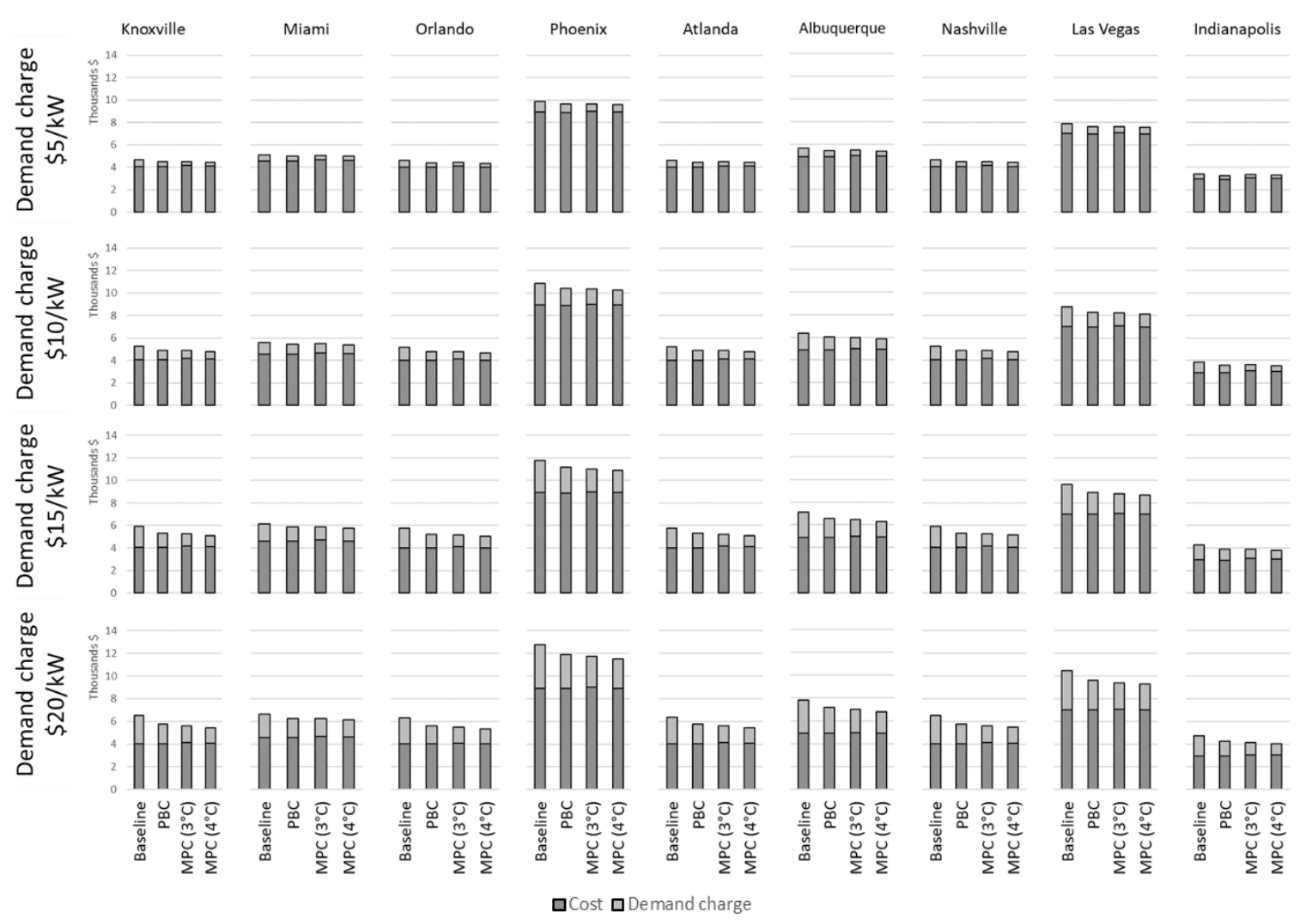

Figure 15: Total electricity cost for AC for 50 homes in different cities.

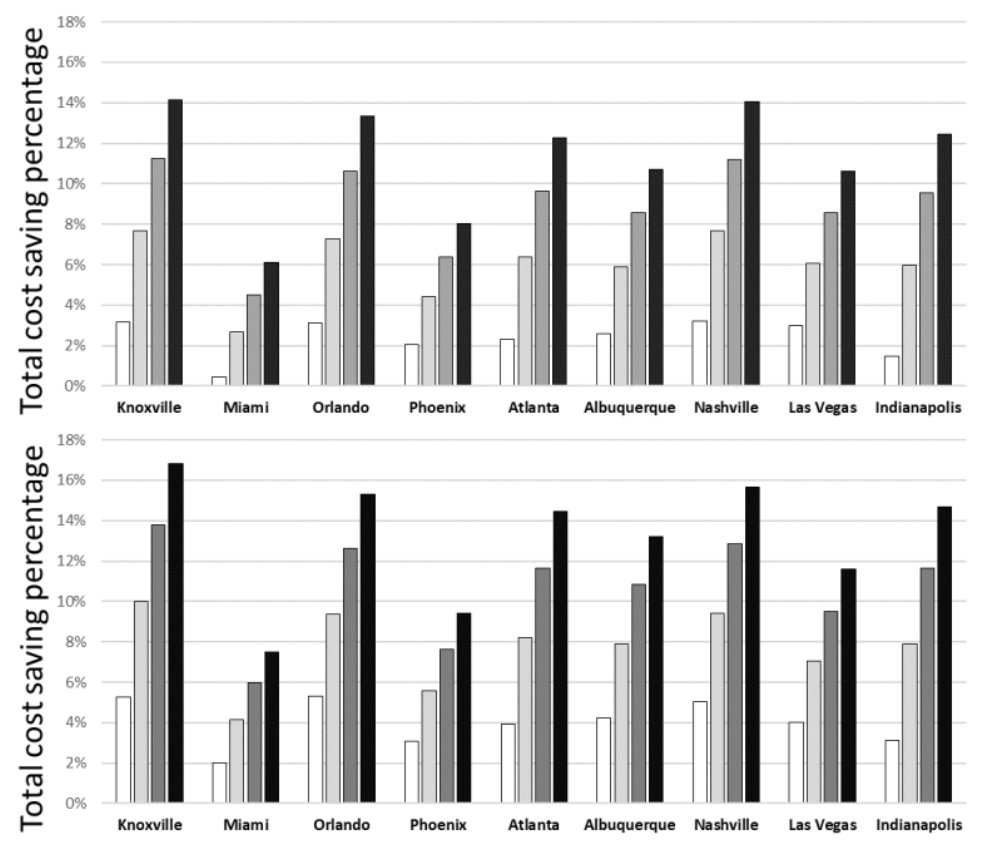

$\square$ Demand charge $\$ 5 / \mathrm{kW} \square$ Demand charge $\$ 10 / \mathrm{kW} \square$ Demand charge $\$ 15 / \mathrm{kW}$ a Demand charge $\$ 20 / \mathrm{kW}$

Figure 16: Total cost saving percentage of MPC compared with baseline case with $3^{\circ} \mathrm{C}$ (top) and $4^{\circ} \mathrm{C}$ comfort bounds (bottom). 


\subsection{DISCUSSION}

\subsubsection{Solution Quality}

Simulations in this study are carried out separately for MPC savings (Section 3.4.1) and demand savings (Section 3.4.2). Obviously, there is interaction between the two control strategies that contradicts the savings potential of each other (e.g., MPC savings potential would be offset by demand charge strategy). Appropriate weight between MPC savings and demand savings can be identified that would vary with the outdoor environment in a given whole month. Also, the two strategies could be applied separately; for example, "MPC without demand charge" can be applied for typical summer weather while demand charge only can be implemented for the hottest days.

\subsubsection{MPC and PBC Implementation}

To implement the MPC strategy a significant amount of data is required including the weather forecast, a forecast of building internal heat loads, building model parameters, and characteristics of the AC system. With time, it is expected that much of this data can be learned or trained with minimal sensors (e.g., indoor temperature and historical weather data). Some basic information on the building orientation, roof color, cladding type and color, etc., could also be useful for training the building model. All of these inputs to the MPC are subject to uncertainty and can cause error in the MPC output. This is the major disadvantage of the MPC strategy.

The major benefits of the MPC strategy include the ability to simulate the peak demand limiting event beforehand to determine what the peak load reduction potential is for a given number of homes with any set of allowable deadbands. In this way, the peak load can be planned ahead of time with a reasonable assurance that the temperature limits of the customers will not be exceeded (relying on the accuracy of MPC inputs). Because of this, the on/off schedules of the ACs can be determined hours ahead of the loadlimiting event and be dispatched from an aggregator. There is no need for high-frequency communication between the homes and an aggregator.

MPC is much more computationally expensive than PBC. Optimizations using relatively complex building models and with forecast horizons sufficient to enable precooling of buildings ( 4-6 hours) can require a lot of computing power, particularly when coordinating among many different homes and using intervals of less than five minutes.

To implement the PBC strategy, reliable and continuous communication is required between the homes and an aggregating agent that issues control commands. Each home needs to provide an updated indoor temperature, set point, and $\mathrm{AC}$ state to the aggregator, and the aggregator must provide on/off control commands to the $\mathrm{AC}$ of each house. If a home loses communication with the aggregator, it cannot be issued new commands and therefore is no longer participating in the control. This study used 5-minute and 1-minute intervals for updating and issuing new commands to each $\mathrm{AC}$. The 5-minute interval is consistent with the industry-standard 5-minute off-delay; however, most units do not have minimum limits for the on-time of a cycle.

The main benefit of the PBC strategy is that no building models are required. The only required information is on the power consumption of the $\mathrm{AC}$, the current state of the $\mathrm{AC}$, the cooling set point, and the current indoor temperature. Aside from the AC power, the other values are typically available from the thermostat and just need to be communicated to an aggregate controller. 


\subsubsection{CONCLUSIONS}

In this project, a control-oriented residential building model is developed with actual measurement. Simulation case studies are carried out based on the estimated building model and AC model. Cost savings potentials of MPC are investigated compared with the conventional method (feedback control). Also, considering the residential building cluster (50 houses), demand shedding strategies with PBC and MPC are tested focusing on the target-hour (1-hour duration) and global regulation (all day long). Their potential is discussed in the context of monthly bill cost. The main findings are summarized as follows:

- The averaged cost savings with MPC compared with the baseline control is $10.6 \%$ and varies from 3.2 to $23.3 \%$ with respect to the prediction horizon, comfort bound, and TOU price structure. The savings potential increases with larger comfort bound, longer prediction horizon, and larger on-peak to off-peak price ratios.

- The target-hour demand shedding potential of MPC is $94-99.8 \%$ relative to the baseline (peak power reduction of up to $2 \mathrm{~kW}$ per $\mathrm{AC}$ and energy reduction of up to $1.6 \mathrm{kWh}$ per $\mathrm{AC}$ ), while that of $\mathrm{PBC}$ is about $40 \%$ compared with the baseline.

- In the target-hour load-up simulation, about $85 \mathrm{kWh}(1.7 \mathrm{kWh}$ per AC) of additional electricity can be consumed per hour relative to the baseline.

- The demand shedding potential of PBC ranges from $18.2 \%$ to $31.1 \%$ in each city compared with the baseline case. The demand shedding potential of MPC is $24.7 \%-41.8 \%$ and $25.6 \%-46.0 \%$ in $3{ }^{\circ} \mathrm{C}$ and $4^{\circ} \mathrm{C}$ comfort bounds. For the Knoxville weather case, the demand reduction potential for MPC is $\sim 40 \%$ or $800 \mathrm{~W}$ per AC.

- The monthly total cost reduction percentage with the demand shedding strategy is $2.4 \%-11.3 \%$ and $4.0 \%-13.2 \%$ in $3^{\circ} \mathrm{C}$ and $4^{\circ} \mathrm{C}$ comfort bounds with different demand cost.

\section{CASE STUDY 2: RESIDENTIAL WATER HEATER SYSTEMS}

\subsection{WATER HEATER MODEL}

The water heater is thermally modeled as a 2-node model split into upper and lower halves. A network of thermal resistances and capacitances is used to model the heat transfer in the water heater, as depicted in Figure 17. During hot water draws, water is removed from the upper node, which is replaced with water from the lower node (at temperature $T_{\text {low }}$ ). The water that moves from the lower node to the upper node is replaced with cold water (at temperature $\mathrm{T}_{\text {cold }}$ ). If the temperature of the lower node is ever calculated to be higher than that of the upper mode, then that time step of the simulation is rerun using a single-node model. This is done to model buoyancy-driven advection caused by temperature differentials in the tank. Since this phenomenon happens very quickly, the short time during which warmer water is located below colder water can be neglected, and a single-node model is sufficiently accurate (also depicted in Figure 17). Equations 16-18 are used to calculate the change in temperature of the water heater nodes.

$$
\begin{gathered}
T_{u p}(t+1)=T_{\text {up }}(t)+\frac{\Delta t}{C_{u p}}\left(\frac{T_{\text {ambient }}-T_{u p}}{R_{u p}}+\dot{V} \rho c_{p}\left(T_{\text {low }}-T_{\text {up }}\right)\right) \text { for } T_{\text {up }}(t+1) \geq T_{\text {low }}(t+1), \\
T_{\text {low }}(t+1)=T_{\text {low }}(t)+\frac{\Delta t}{C_{\text {low }}}\left(\frac{T_{\text {ambient }}-T_{\text {low }}}{R_{\text {low }}}+\dot{V} \rho c_{p}\left(T_{\text {cold }}-T_{\text {low }}\right)\right) \text { for } T_{\text {up }}(t+1) \geq T_{\text {low }}(t+1), \\
T_{\text {avg }}(t+1)=T_{\text {avg }}(t)+\frac{\Delta t}{C_{\text {low }}+C_{\text {up }}}\left(\left(T_{\text {ambient }}-T_{\text {avg }}\right)\left(\frac{1}{R_{\text {low }}}+\frac{1}{R_{u p}}\right)+\dot{V} \rho c_{p}\left(T_{\text {cold }}-T_{\text {avg }}\right)\right),
\end{gathered}
$$


where $\mathrm{R}_{\text {up }}=1.6 \mathrm{~K} / \mathrm{W}, \mathrm{R}_{\text {low }}=1.6 \mathrm{~K} / \mathrm{W}, \mathrm{C}_{\text {up }}=300 \mathrm{~kJ} / \mathrm{K}, \mathrm{C}_{\text {low }}=300 \mathrm{~kJ} / \mathrm{K}, \mathrm{T}_{\text {cold }}=15.6^{\circ} \mathrm{C}, \Delta \mathrm{t}=300 \mathrm{~s}, \dot{V}$ is the cold-water flow rate entering the water heater in $\mathrm{m}^{3} / \mathrm{s}, \rho$ is the density of cold water in $\mathrm{kg} / \mathrm{m}^{3}$, and $\mathrm{c}_{\mathrm{p}}$ is the specific heat capacity of cold water in $\mathrm{J} / \mathrm{kg}-\mathrm{K}$.

The controls of the water heater are modeled to match those of a typical electric resistance water heater. The lower element has a $5.6^{\circ} \mathrm{C}\left(10^{\circ} \mathrm{F}\right)$ deadband, where the element is turned on when the temperature falls below the set point minus the deadband and turns off when the temperature increases above the set point. The upper element has a deadband of $8.3^{\circ} \mathrm{C}\left(15^{\circ} \mathrm{F}\right)$ and follows the same controls. Only one element can be energized at any point in time, with the upper element having operational priority over the lower element.

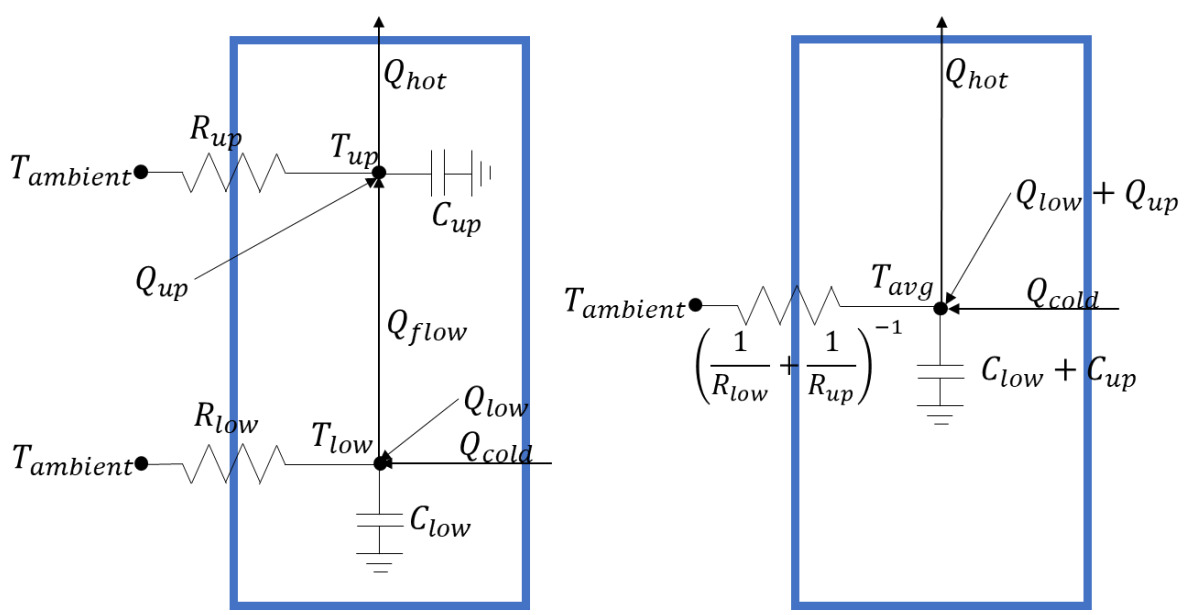

Figure 17: Thermal model of water heater: (left) 2-node model; (right) 1-node model.

\subsection{HOURLY LOAD FLEXIBILITY}

To quantify the flexibility of residential water heaters, hour-long load shed and load-up periods are simulated for all hours of the day. The load shed and load-up results are analyzed using two metrics: the peak power change and energy flexibility potential. The peak power flexibility is the difference between the 5-minute peak power use during the shed/load-up hour of the baseline and the PBC or MPC cases. The energy reduction potential is the difference between the energy use during the shed/load-up hour for the baseline and the PBC or MPC cases. Since the load-up condition is typically associated with unexpected overgeneration, only the $\mathrm{PBC}$ is evaluated for that case.

Measured hot water use data from 50 occupied homes is used in the simulations. Four different days are analyzed, Sunday through Wednesday for a week in January. As seen in Figure 18, the hot water use profile is similar for the weekdays and the use on Sunday is significantly different. Therefore, the results will show average load reduction for weekdays using the Monday through Wednesday data and the weekend results will be for the Sunday data. 


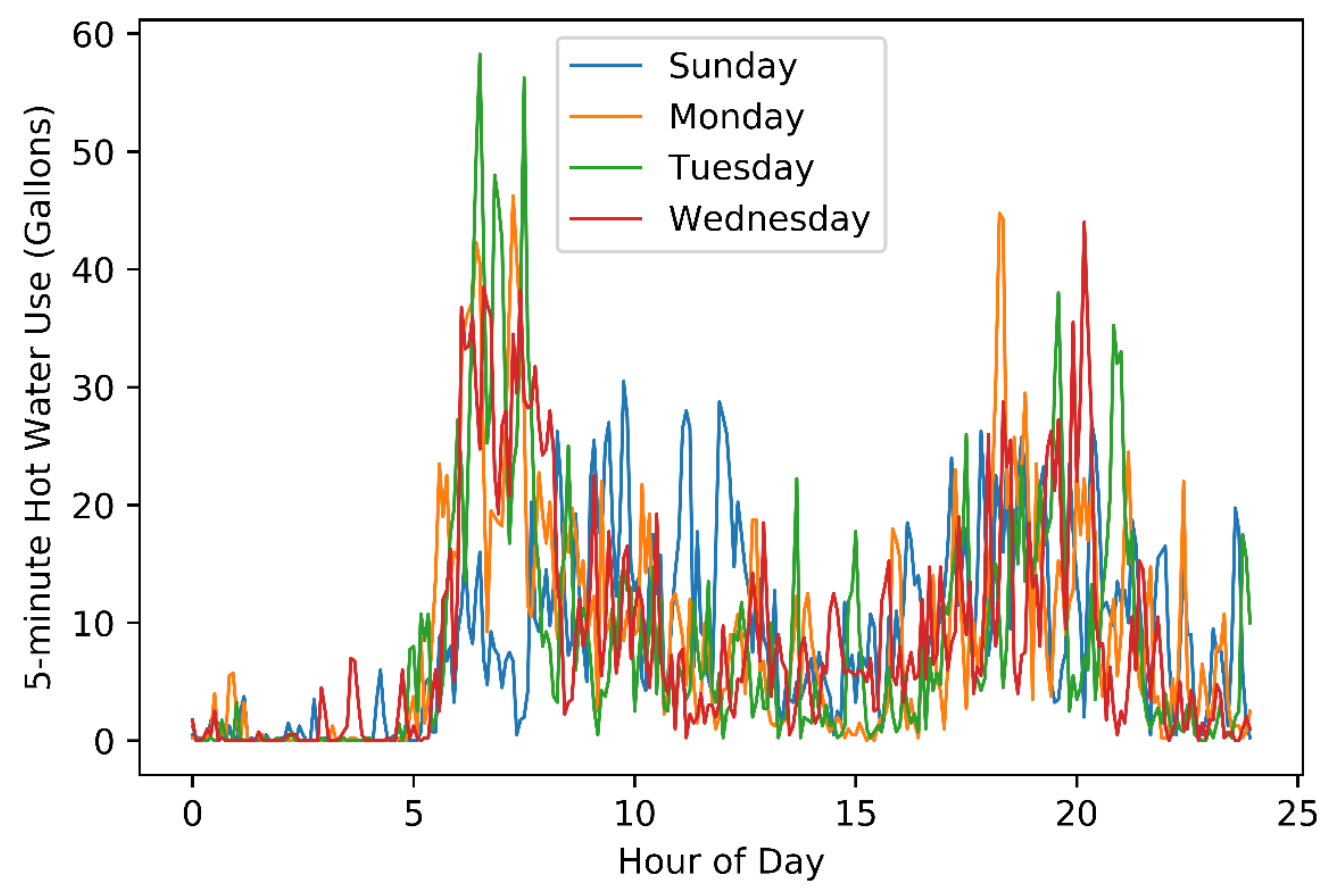

Figure 18: Aggregate hot water use for 50 occupied homes on different days.

\subsubsection{PBC Implementation-Load Shed}

PBC was implemented for each hour of the day (e.g., a 1-hour shed was called for from 6:00 a.m. to 7:00 a.m. for one simulation, and another simulation was run with a 1-hour shed from 7:00 a.m. to 8:00 a.m.). The initial trial for each hour was to turn off the lower element for all units during the load-shed hour. If the upper elements for any of the units turned on, then the trial was unsuccessful. Following an unsuccessful trial, the number of water heaters allowed on during any time step was increased by one and PBC was implemented under these constraints. This process was repeated until the minimum number of units were running each hour while not requiring additional upper element use relative to the baseline. Additionally, the peak power use at any time of the day was limited to the baseline peak. This was done to reduce the size of the rebound peak following the shed. Given the diverse use cases for load flexibility, a single optimal control methodology for controlling the rebound peak is not available. It may be desirable to further limit the rebound peak, which would result in decreased flexibility.

\subsubsection{PBC Implementation-Load-Up}

Similar to the load-shed case, $\mathrm{PBC}$ was implemented for each hour of the day for the load-up case. For the initial trial, all water heaters were turned on during the load-up hour. If this power level could not be maintained for the entire hour, then the number of water heaters that were required to be on during the shed hour was reduced by one. This process continued until a consistent power level was achieved for the load-up hour. Note that in some cases this resulted in a reduced peak power during the load-up hour relative to the baseline because we were looking for constant power use over the entire hour. 


\subsubsection{MPC Implementation-Load Shed}

The MPC used a linear, mixed-integer optimization, so the two-node water heater model was simplified to a single-node model to keep the problem linear. While the MPC was performed on a single-node model, the control decisions from the MPC were then simulated using the two-node water heater model. The MPC was trying to minimize the objective function shown in Equation 19 subject to the constraining Equations 20-34. The MPC was run every hour with a forecast horizon of four hours. In this way, the MPC dispatched controls for an hour based on a predictive simulation over the next four hours. This ensured that the MPC simulation included time intervals both before and after a shed hour to allow for an appropriate amount of preheating to reduce the likelihood of having too-cold water in the water heaters at the end of the shed.

$$
\begin{gathered}
\text { Obj }=\sum_{t, \text { shed }} \sum_{w h} W_{1} \alpha(t) P(t, w h)+W_{2} T_{\text {max }}^{*}(t, w h)+W_{3} T_{\text {min }}^{*}(t, w h)+W_{4} T_{\text {target }}^{*}(t, w h), \\
T_{\text {max }}^{*}(t, w h) \geq T_{\text {avg }}(t, w h)-T_{\text {max }}(w h), \\
T_{\text {max }}^{*}(t, w h) \geq 0, \\
T_{\text {min }}^{*}(t, w h) \geq T_{\text {min }}(w h)-T_{\text {avg }}(t, w h), \\
T_{\text {min }}^{*}(t, w h) \geq 0, \\
T_{\text {target }}^{*}(t, w h) \geq T_{\text {target }}(w h)-T_{\text {avg }}(t, w h), \\
T_{\text {target }}^{*}(t, w h) \geq 0, \\
T_{\text {min }}(w h)=0.5\left(T_{\text {set }}(w h)+T_{\text {cold }}\right), \\
T_{\text {target }}(w h)=\left(T_{\text {set }}(w h)+0.5 \text { Deadband }{ }_{\text {low }}\right), \\
M a x\left(\sum_{w h} P(t, w h)\right) \leq M a x\left(\sum_{w h} P_{\text {baseline }}(t, w h)\right),
\end{gathered}
$$

where $T_{\max }$ is the maximum temperature the water heater is allowed to heat the water, $T_{\min }$ is the lowest average tank temperature desired, and $T_{\text {target }}$ is the lowest temperature before heating should be turned on.

An MPC optimization was run every hour for a four-hour period, which ensured that the MPC had ample time to preheat before the shed periods. The MPC was run every hour to provide better performance by dispatching only the first hour of the four-hour optimization. This is because the decisions made in the latter half of the optimization cannot take into account anything beyond the four-hour period and can therefore leave the water heaters in a state that is not optimal for the upcoming hot water use. Therefore, dispatching control decisions from the first quarter of the optimization eliminates this issue.

\subsection{DEMAND REDUCTION}

In addition to evaluating the hourly load flexibility of water heaters, the demand reduction capability of water heaters is also analyzed. In the demand reduction approach, the aggregate power use of the water heaters is limited over the entirety of the day. As the peak demand is reduced, the valleys tend to be filled providing a load-leveling effect. 


\subsubsection{PBC Implementation-Demand Reduction}

When implementing the demand reduction strategy with PBC, simulations were run to find the minimum number of water heaters that could be operated at a time. The PBC is only allowed to control the lower water heater elements, so if the upper element turned on because of low tank temperatures, the minimum number of water heaters allowed on was increased by one.

\subsubsection{MPC Implementation-Demand Reduction}

The MPC objective function was modified to achieve a power limit across the entire day. This was achieved by adding a variable that was set to zero if the number of units operating at any time step was below the desired limit and a value equal to the difference between these numbers if more units than desired were operating. This variable was then included in the objective function to be minimized. In this way, the MPC was not penalized for using any power up until the targeted power limit. Above the power limit, the MPC objective function was penalized. This constraint is shown in Equations 29 and 30 and in the objective function in Equation 31.

$$
\begin{gathered}
\operatorname{WHon}^{*}(t) \geq \sum_{w h} \operatorname{On}(t, w h)-N, \\
W \operatorname{Hon}^{*}(t) \geq 0, \\
C \sum_{t} \operatorname{WHon}^{*}(t),
\end{gathered}
$$

where WHon* is the number of water heaters operating above the target number, $\mathrm{N}$, and $\mathrm{C}$ is a weighting factor for the objective function.

\subsection{WATER HEATING SIMULATION RESULTS}

\subsubsection{Baseline Operation}

The baseline operation of the fleet of 50 water heaters was simulated for the four days of hot water draw data. The power consumption of the fleet is shown in Figure 19. As with the hot water use, the power use profile of the water heaters on weekdays is similar, while the weekend day (Sunday) is significantly different. The maximum possible power consumption of the fleet of water heaters is shown with the black dashed line to illustrate that at any point in time the maximum number of water heaters that are on is approximately one-third of the fleet. The power consumption, and therefore the power reduction potential, of the water heaters is very low during the early morning and late evening hours. Power consumption is highest during the morning hours when many people take showers. 


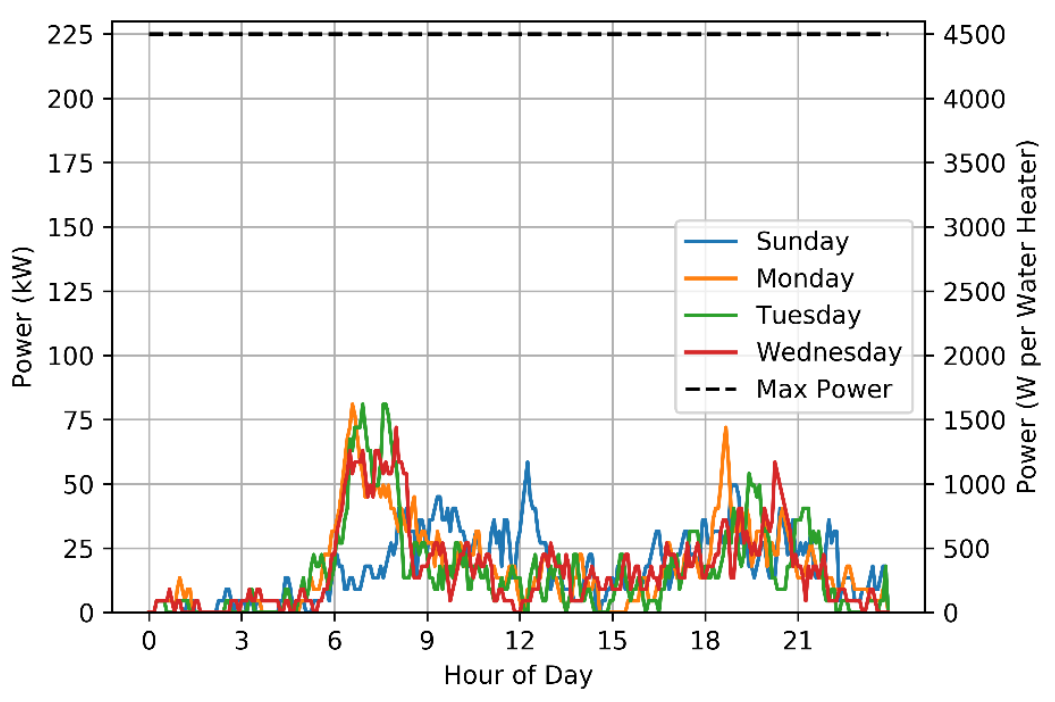

Figure 19: Aggregate power use of 50 water heaters on different days.

\subsubsection{Hourly Flexibility}

\subsubsection{Priority-based control}

As mentioned in Section 4.2, simulations were run to limit the peak power for each hour of the day using the PBC. Sample plots showing the results for a shed from 6:00 a.m. to 7:00 a.m. and from 6:00 p.m. to 7:00 p.m. on a Monday are shown in Figure 20. Likewise, results for a load-up for the same two time periods on the same day are shown in Figure 21. 

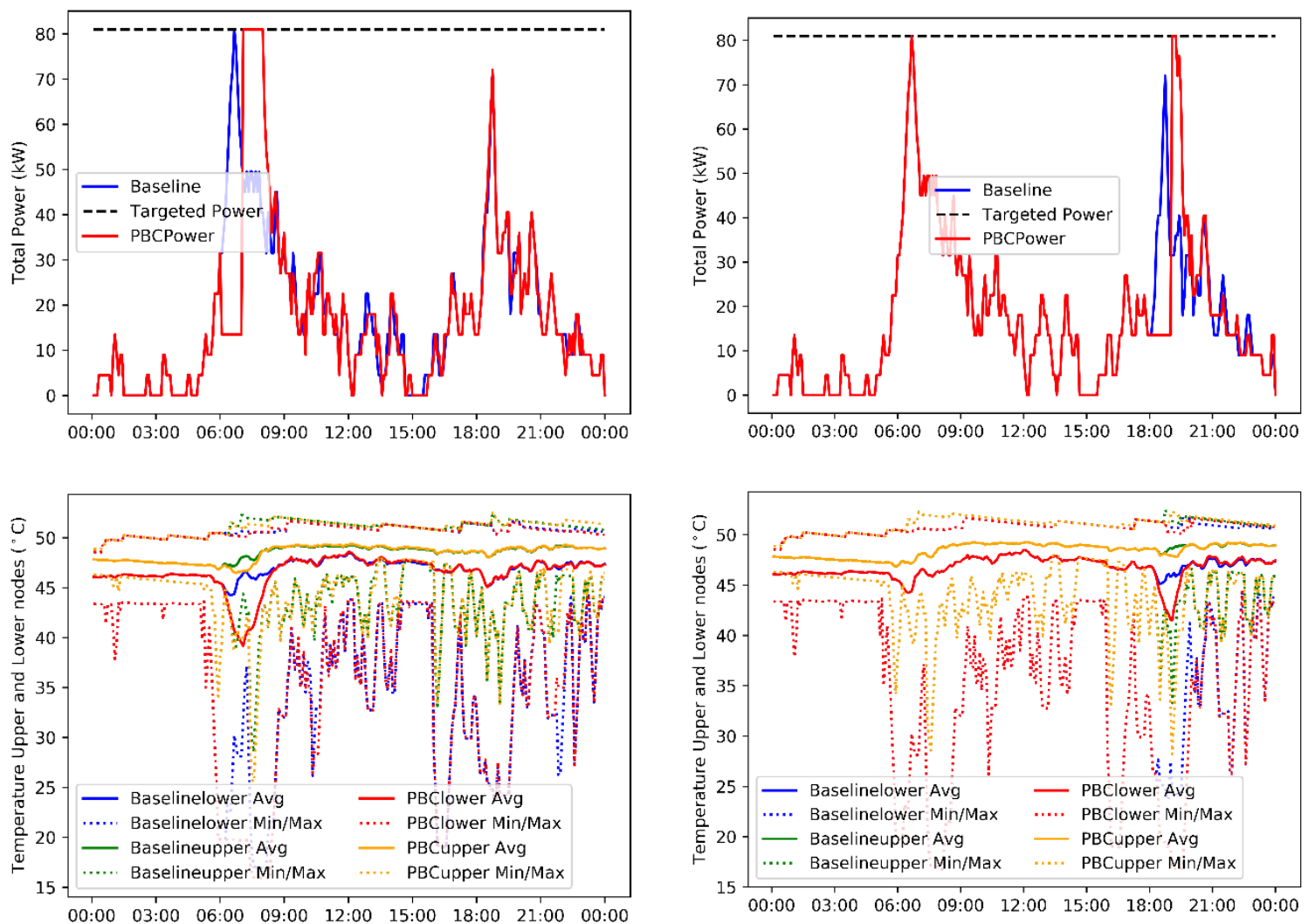

Figure 20: Power and temperature profiles for baseline and $\mathrm{PBC}$ with shed periods of 6:00 a.m. to 7:00 a.m. (left) and 6:00 p.m. to 7:00 p.m. (right) for Monday. 

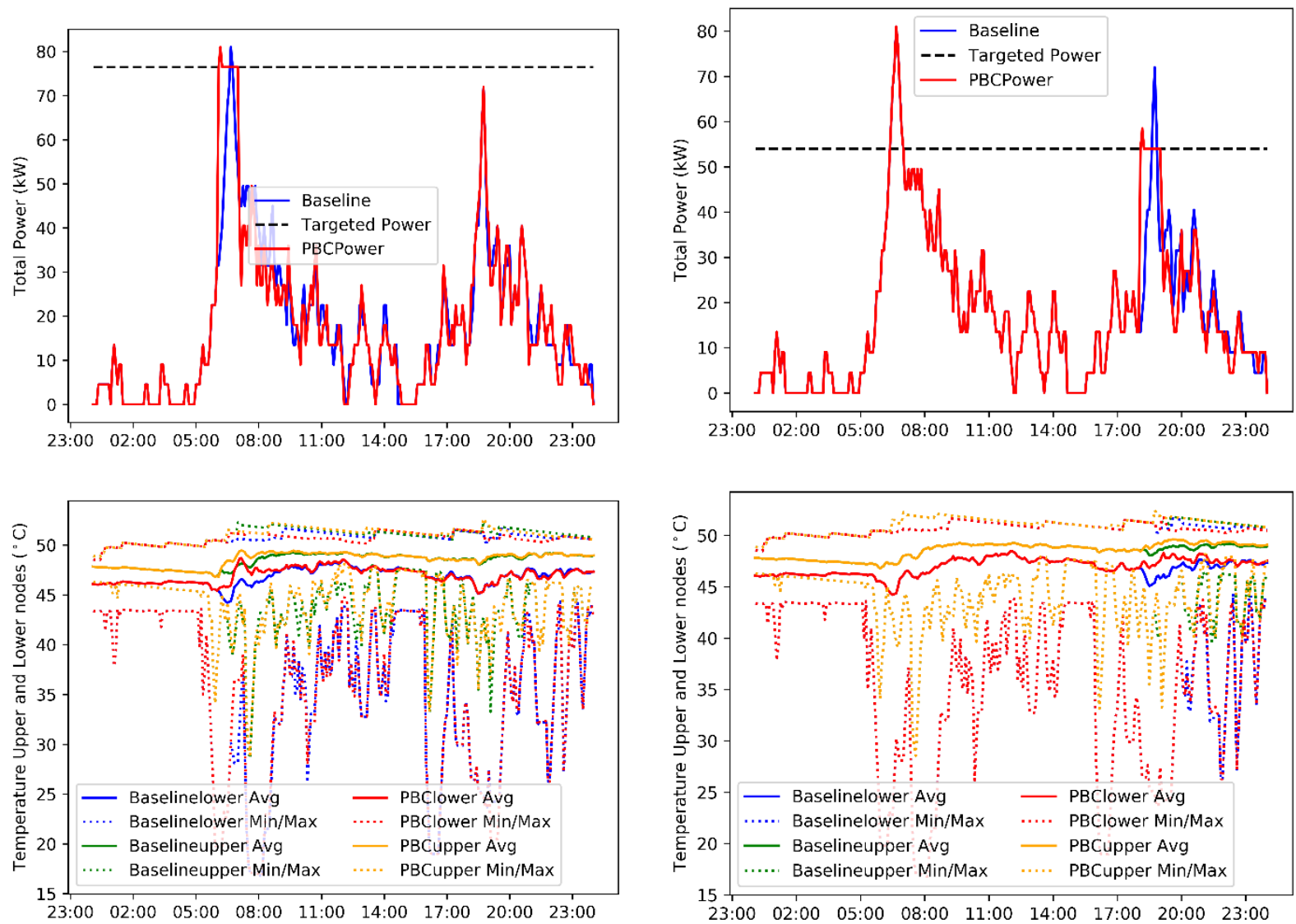

Figure 21: Power and temperature profiles for baseline and PBC with load-up periods of 6:00 a.m. to 7:00 a.m. (left) and 6:00 p.m. to 7:00 p.m. (right) for Monday.

\subsubsection{Model-predictive control}

As mentioned in Section 4.2, simulations were run to limit the peak power for each hour of the day using MPC. Sample plots showing the results for a shed from 6:00 a.m. to 7:00 a.m. and 6:00 p.m. to 7:00 p.m. on a Monday (the same time period as those shown in Figure 20 for PBC) are shown in Figure 22. As with the PBC case, the MPC limited the rebound peak to no higher than the baseline peak power. A small spike of power before the shed hour can also be seen as the MPC preheats the water in some of the water heaters.

\subsubsection{Comparative results}

Focusing on the load-shed or load-up hour, we can show the variations in peak power and energy flexibility with time. Since the hot water use pattern varies between weekdays and weekends, results are shown separately for these two types of days. Results for peak power flexibility for weekdays are shown in Figure 23 using units of watts per water heater. This is an average peak power reduction for the hour that can be expected from a large fleet of water heaters. In addition to the PBC and MPC peak power reduction results, the maximum possible reduction is also plotted. The maximum peak power reduction corresponds to the baseline peak power use and is useful for determining the percent reduction relative to the baseline. The peak power reduction potential is largest during hours of high hot water use since more units are running and are therefore available to be turned off. However, the MPC and PBC achieve a smaller percentage reduction during these hours because some water heaters must stay on to meet the hot 
water demands of the occupants. Because of the high heating capacity of the water heater element relative to storage size, there is not much increase in sustained peak power available.
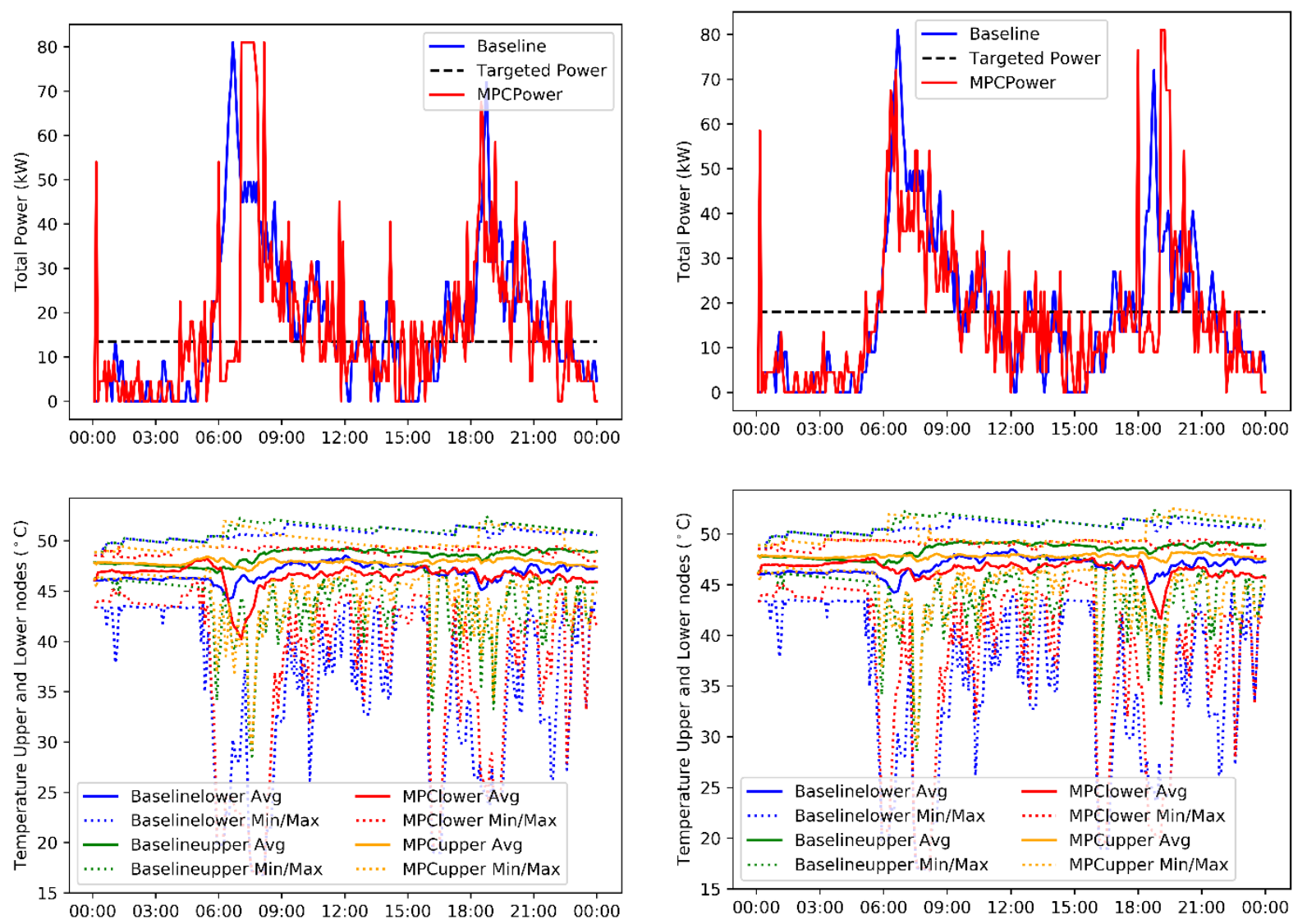

Figure 22: Power and temperature profiles for baseline and MPC with shed periods of 6:00 a.m. to 7:00 a.m. (left) and 6:00 p.m. to 7:00 p.m. (right).

Figure 24 shows the weekday energy flexibility for each hour of the day in terms of watt-hours per water heater. This is the expected energy use increase or decrease that can be expected over each hour for a fleet of water heaters. As with the peak power, the reduction is largest during periods of high hot water consumption. Conversely, energy use can be increased the most during periods with low hot water use.

The results for weekends are shown in Figure 25 for peak power and Figure 26 for energy. 


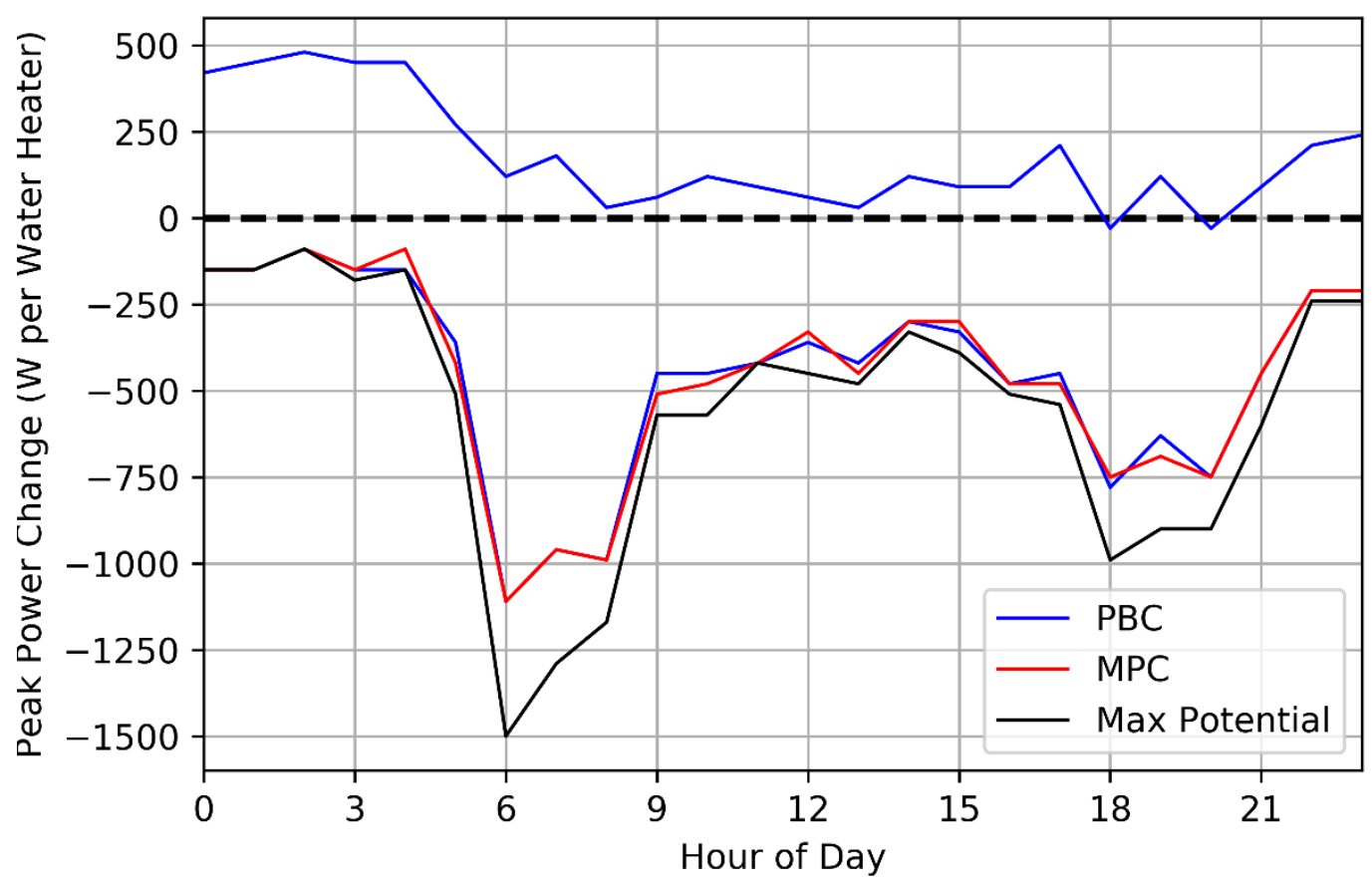

Figure 23: Weekday peak power flexibility for PBC and MPC for each hour of the day.

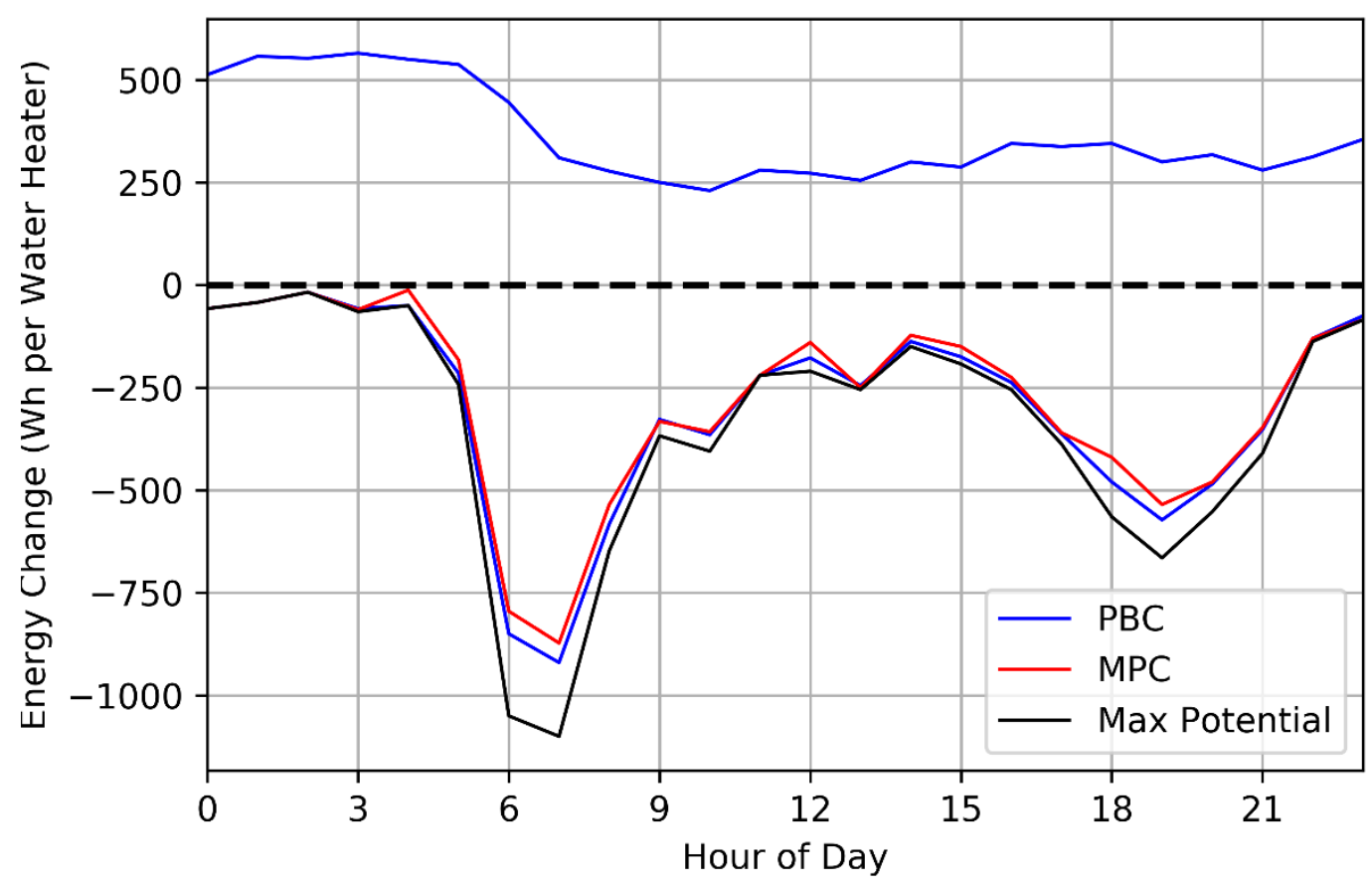

Figure 24: Weekday energy flexibility for PBC and MPC for each hour of the day. 


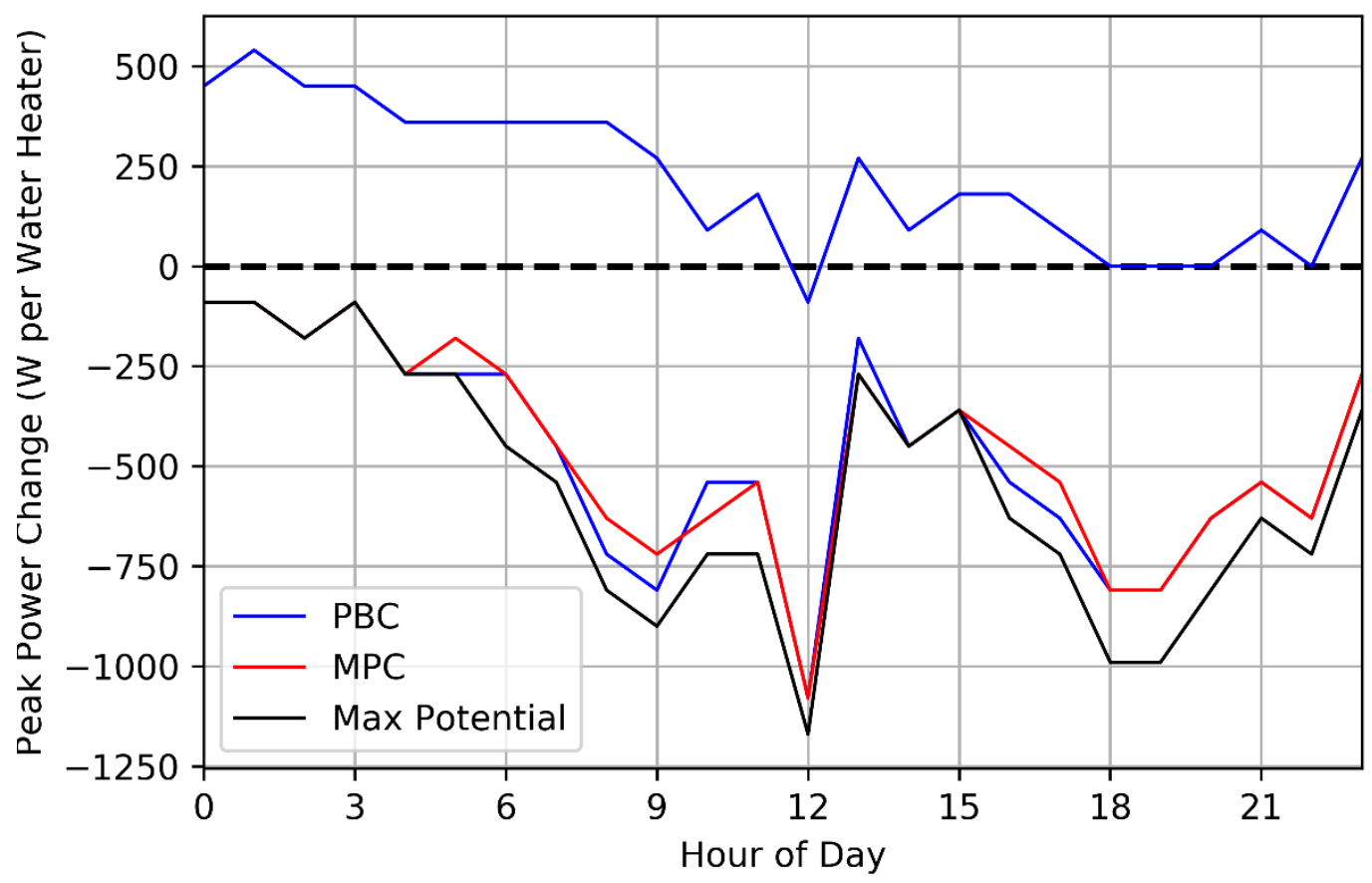

Figure 25: Weekend peak power flexibility for PBC and MPC for each hour of the day.

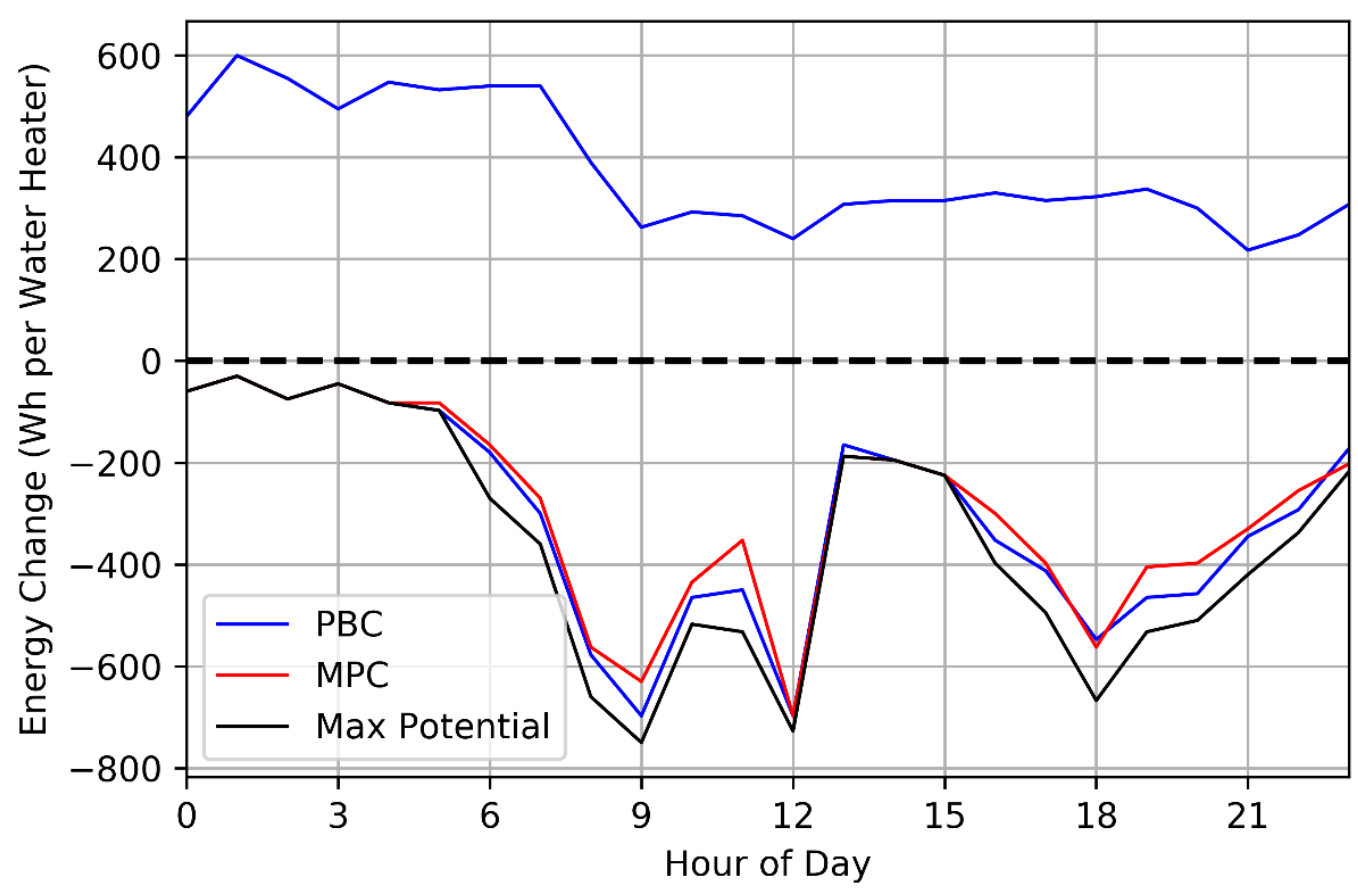

Figure 26: Weekend energy flexibility for PBC and MPC for each hour of the day. 


\subsubsection{Demand Reduction}

\subsubsection{Priority-based control}

As described in Section 4.3.1, PBC was implemented to reduce the daily peak power use of the fleet of water heaters. The PBC results for Sunday and Monday are shown in Figure 27. The PBC is able to greatly reduce the peak power use relative to the baseline with a $72 \%$ reduction $(720 \mathrm{~W}$ per water heater) for Sunday and a $62 \%$ reduction $(1170 \mathrm{~W}$ per WH) for Monday. Note that while the lower tank temperatures are extremely low, with some WHs having no hot water left at the bottom of the tank, the average upper tank temperatures are still very close to the baseline. This is because of the stratification of hot water that naturally exists due to buoyancy forces. The stratification is typically maintained in a tank except under very high hot water draw rates, which could cause the incoming cold water to mix with layers of hot water higher up in the tank. Even with high average temperatures, there were still some homes that had upper tank water temperatures dip significantly below the baseline. This indicates that some homeowner's may have experienced colder than usual hot water in this simulation, and therefore the reduction levels are to be considered aggressive values. Results for the other weekdays were similar to the results shown for Monday.
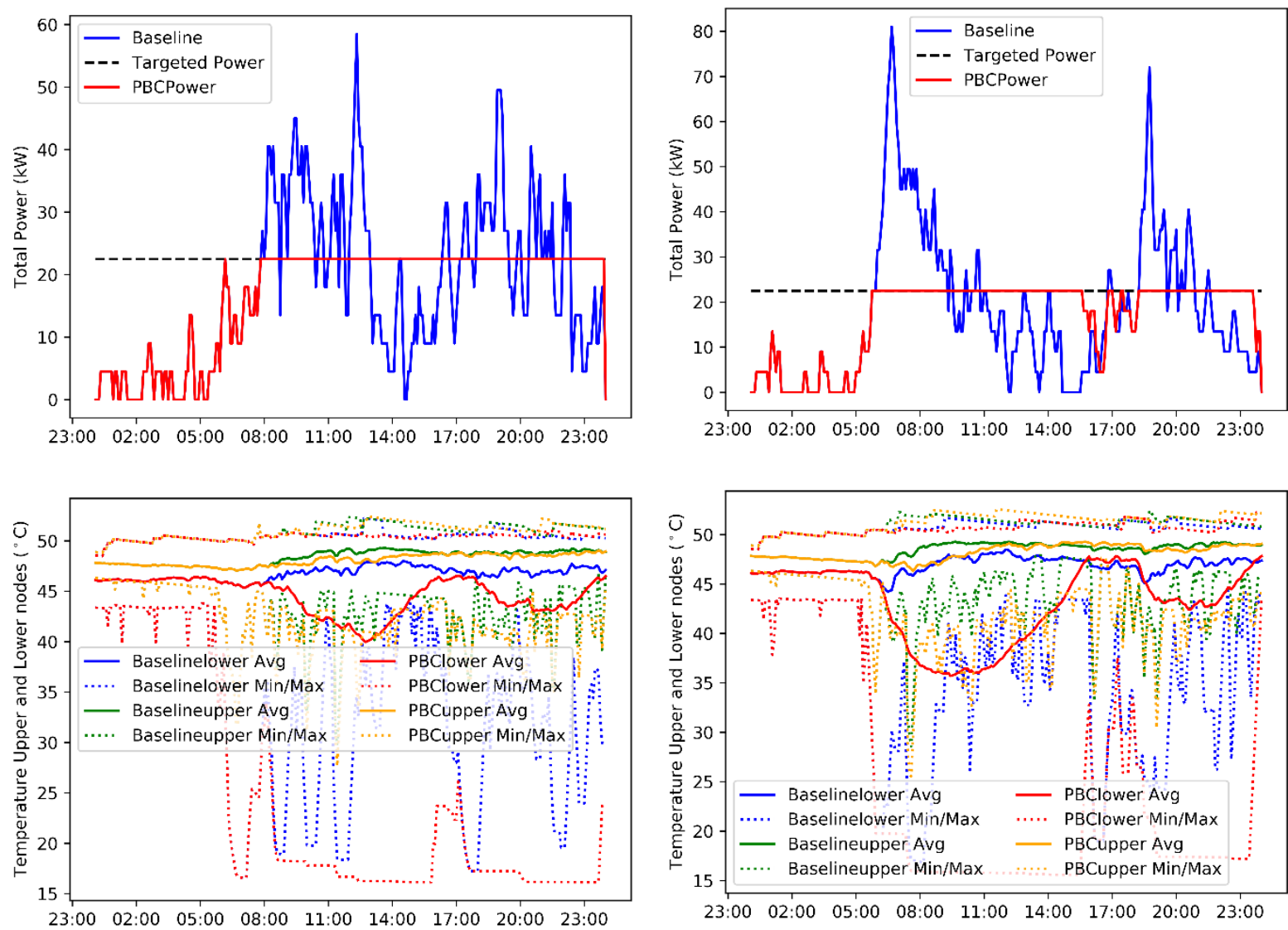

Figure 27: PBC results for demand reduction for Sunday (left) and Monday (right).

\subsubsection{Model-predictive control}

Results for the MPC for demand reduction on Sunday and Monday (matching plots shown for PBC) are shown in Figure 28. It can be seen that the power use sometimes exceeded the target power level for the MPC. This is due to the soft constraint that was used to limit the power. To keep the average tank 
temperature higher, additional units were turned on above the target power limit. The MPC achieved a $46 \%$ reduction in peak power $(630 \mathrm{~W}$ per $\mathrm{WH})$ for Sunday and a $44 \%$ reduction $(900 \mathrm{~W}$ per $\mathrm{WH}$ ) for Monday. One challenge for the MPC is that it is using only a single-node model with a single heating element for its optimization. This means that all constraints are based on an average tank temperature and using an element that heats the entire tank. To achieve the highest reduction, only the top half of the tank could be heated using the upper element, reducing the volume of water that is heated, and also the power consumption. This behavior can be seen in the PBC results and contribute to its higher demand reduction results.

Simulations were also run that allowed the water heater to increase the tank temperature up to $140^{\circ} \mathrm{F}$. This allows the MPC to preheat the water more before hot water draws to maintain the water at more acceptable temperatures for occupants. As seen in Figure 29, the MPC achieves the same level of power reduction as the $\mathrm{PBC}$ (with the exception of one or two intervals) but with much higher tank temperatures. The power profile is also generally flatter because of the additional power use to preheat the water before the morning hot water usage peak.
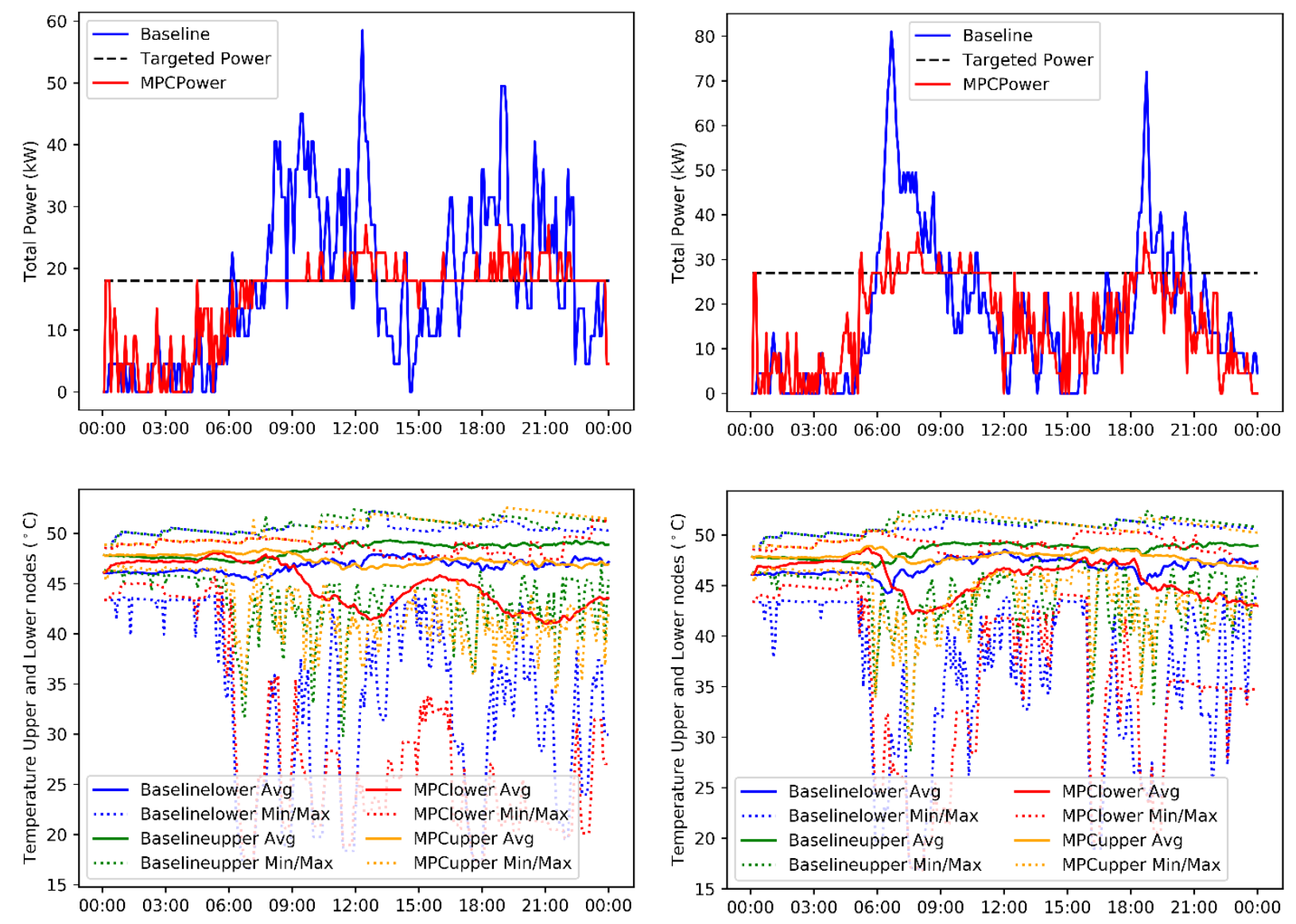

Figure 28: MPC results for demand reduction for Sunday (left) and Monday (right). 

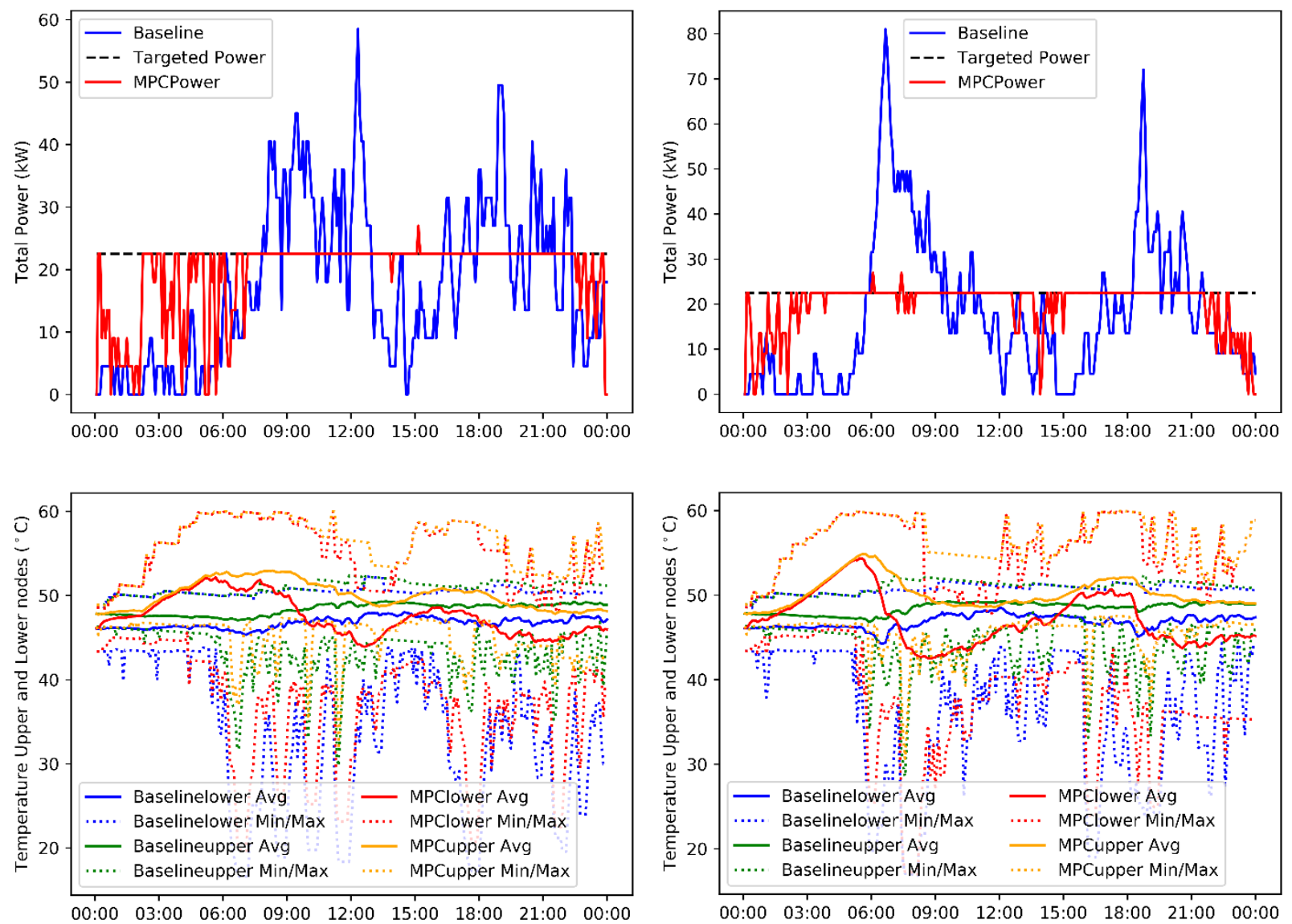

Figure 29: MPC results for demand reduction for Sunday (left) and Monday (right) with a maximum water temperature of $60^{\circ} \mathrm{C}\left(140^{\circ} \mathrm{F}\right)$.

\subsection{DISCUSSION}

The peak power and energy reduction potential of water heaters are directly related to the hot water consumption profile of homeowners. This results in larger power reduction potential during periods of high hot water consumption such as mornings and evenings during weekdays. The highest power consumption period for water heaters is weekday mornings when many people take showers. However, during this period there are still only approximately one-third of the water heaters running during any 5minute interval. This results in the maximum possible peak power reduction on a per-water-heater basis being $\sim 1,500 \mathrm{~W}$ (relative to a power rating of 4,500 W). During hours of the day outside of the highest hot water consumption, the maximum peak power reduction is significantly less.

Both MPC and PBC achieved power and energy reduction of the shed hour very close to the maximum values, 70\%-100\%. The MPC generally maintains higher hot water temperatures because of its ability to preheat some water heaters before the shed. However, the difference in peak power and energy reduction between PBC and MPC is typically small at $10 \%$ or less. Energy reduction during the shed hour is also similar for the PBC and MPC with $900 \mathrm{Wh}$ per water heater available during the weekday morning peak. 
Water heaters have limited ability to provide a sustained (i.e., over the span of an hour) increase in power use. This is due to their relatively high heating capacity compared with their storage volume. This study used 50-gallon water heaters, which are typical in homes, but there are larger, up to 80 gallon, water heaters available that would increase load-up flexibility. Water heaters can provide large increases in power over short durations (i.e., 5 to 15 minutes). Energy consumption over an hour can be increased by 200-500 Wh per water heater depending on time of day and day of week.

The daily peak demand reduction potential for water heaters is estimated at $600-700 \mathrm{~W}$ per water heater for weekends and 900-1200 W per water heater for weekdays. The largest peak occurs during the morning hours when hot water use is highest, so the most benefit would likely be seen during winter days when there is a coincident peak for electric heating systems (which often use more power than water heaters). The next largest peak is during the early evening hours, which may be beneficial to reduce during summer days when $\mathrm{AC}$ also has a peak in the late afternoon or early evening.

\subsubsection{MPC and PBC Implementation}

PBC achieved very good results when applied to water heaters. The largest challenge associated with the application is ensuring that the control provides sufficient hot water. This study allowed the PBC to turn off the lower element only, leaving the upper element free to operate if required. This approach relies heavily on the assumption that the thermostat controlling the upper element has a setting that maintains an acceptable temperature of water. During typical operation the upper element is rarely used, meaning that the temperature in the tank is mostly controlled by the operation of the lower heating element and thermostat. This issue would likely be eliminated with water heaters that are grid-enabled because they will likely have a singular control board controlling both elements based on a single set point unlike traditional water heaters that have two separate thermostats.

The MPC was implemented with a single-node tank model to avoid the nonlinear behavior of natural convection and be compatible with a linear optimizer. The results for the MPC could likely have been improved with the use of a two-node model and control of both heating elements. This would have required a nonlinear optimizer that would have increased computational time and is not guaranteed to find the globally optimal solution. The water heater models are relatively simple and are not much of an obstacle for MPC; however, forecasts of hot water use can be more challenging. Research has been done on using machine learning to learn the pattern of water heater use, which is a reasonable proxy for hot water use [9].

\subsubsection{Conclusions}

This study evaluated the load flexibility of a fleet of 50 water heaters that were simulated using two-node models and hot water draw profiles measured from occupied homes. Two control and coordination approaches were used, PBC and MPC, and the flexibility was analyzed on an hour-by-hour basis and as overall peak reduction. Main findings are summarized in the following:

- The hourly power reduction potential follows a pattern that closely matches that of hot water use. This results in the highest load reduction potential during weekdays from 6:00 a.m. to 9:00 a.m. The peak power reduction is $\sim 1,000 \mathrm{~W}$ per water heater, and the energy reduction is $\sim 800 \mathrm{Wh}$ per water heater.

- The hourly power increase potential is highest during the night and early morning when there is little hot water use but there is still an opportunity for the water heaters to be turned on to recover from tank losses. The maximum sustained power increase over an hour is $\sim 500 \mathrm{~W}$ per water heater for the midnight to 4:00 a.m. period. The energy use increase over the hour for this time frame is $\sim 500 \mathrm{Wh}$ 
per water heater. During other hours of the day this falls to $250 \mathrm{Wh}$ per water heater. The power increase potential is similar for weekdays and weekends.

- The daily demand reduction potential for water heaters is $\sim 900-1,200 \mathrm{~W}$ per water heater for weekdays and 600-700 W per water heater for weekends.

- Both PBC and MPC performed very well when controlling a fleet of water heaters to achieve a desired power change. It is likely that simple control methodologies like PBC will be easier to implement and provide similar results to more advanced algorithms.

\section{CASE STUDY 3: SUPERMARKET REFRIGERATION SYSTEM}

In the reference system, three compressors, including two fixed-speed compressors (models 4MTC-7K and 4MTC-10K) and one variable-speed compressor (model 4MTC-10K_V), are used to handle the cooling demand from the open-case refrigerator display cases. They have similar rated capacities and powers, as listed in Table 6. The variable-speed compressor is the same model as the fixed-speed compressor (4MTC-10K), but it is being powered by a variable frequency drive. The compressors are connected to a single open display case and a false load loop that can be used to simulate additional cases. The rated cooling capacity of the display case is $2,810 \mathrm{~W}$. The following subsections introduce the modeling work of the display case and compressors, simulation assumptions, PBC introductions for both load-shed and load-up scenarios, analysis of simulation results, and discussion.

Table 6: Compressor specifications

\begin{tabular}{cccccc}
\hline $\begin{array}{c}\text { Compressor } \\
\text { type }\end{array}$ & $\begin{array}{c}\text { Temperature } \\
\text { level }\end{array}$ & Model name & $\begin{array}{c}\text { Capacity } \\
\text { control }\end{array}$ & $\begin{array}{c}\text { Evaporator } \\
\text { capacity }(W)\end{array}$ & $\begin{array}{c}\text { Rated } \\
\text { power }(\mathbf{W})\end{array}$ \\
\hline Reciprocating & $\begin{array}{c}\text { Medium } \\
\text { temperature } \\
\text { Medium } \\
\text { Reciprocating }\end{array}$ & 4MTC-10K_V & Variable speed & 11,100 & 9,660 \\
temperature & 4MTC-10K & Fixed speed & 11,400 & 9,720 \\
Reciprocating & $\begin{array}{c}\text { Medium } \\
\text { temperature }\end{array}$ & 4MTC-10K & Fixed speed & 11,300 & 9,400 \\
\hline
\end{tabular}

\section{1 $\mathrm{CO}_{2}$ OPEN-CASE REFRIGERATOR DISPLAY CASE THERMAL MODEL}

A simplified grey-box refrigerator case thermal model was developed, and parameters were trained based on measured data from the medium temperature supermarket carbon dioxide $\left(\mathrm{CO}_{2}\right)$ refrigeration system. The refrigerator case model is thermally modeled as a three-node model. As shown in Figure 30, a 3R3C model is proposed. It is in an electrical analogue pattern with resistance $(\mathrm{R}, \mathrm{K} / \mathrm{W})$ and capacitance $(\mathrm{C}$, $\mathrm{J} / \mathrm{K})$. The physical properties of the refrigeration case affecting thermal transfer are mainly those of the case envelope, internal mass (i.e., food), and air within the case, which are handled separately in this model. The developed model can therefore reflect thermal status and the response of different components in the refrigeration case. It is worth noting that only sensible load is considered in this model. Meanwhile, all Rs and Cs are assumed to be time-invariant. The heat transfer in the case model is described using the first-order differential equations shown in Equations 32-34.

$$
C_{\text {air }} \frac{d T_{\text {ave }}(t)}{d t}=\frac{T_{\text {amb }}(t)-T_{\text {ave }}(t)}{R_{\text {infil }}}+\frac{T_{\text {food }}(t)-T_{\text {ave }}(t)}{R_{\text {food }}}+\frac{T_{\text {case }}(t)-T_{\text {ave }}(t)}{R_{\text {case }}}+C 1 Q_{A C, i},
$$




$$
\begin{gathered}
C_{\text {food }} \frac{d T_{\text {food }}(t)}{d t}=\frac{T_{\text {ave }}(t)-T_{\text {food }}(t)}{R_{\text {food }}}, \\
C_{\text {case }} \frac{d T_{\text {case }}(t)}{d t}=\frac{T_{\text {ave }}(t)-T_{\text {case }}(t)}{R_{\text {case }}}+C 1 Q_{A C, m},
\end{gathered}
$$

where, $C_{\text {air }}, C_{\text {food }}$, and $C_{\text {case }}$ are the thermal capacitances of air in the case, food, and refrigerator case respectively. $R_{\text {infil }}, R_{\text {food }}$, and $R_{\text {case }}$ are the thermal resistance of air infiltration, food-to-air heat transfer, and refrigerator case, respectively. $T_{a m b}$ is the ambient temperature $\left({ }^{\circ} \mathrm{C}\right)$ (i.e., the indoor air temperature surrounding the display case). $T_{\text {ave }}$ is the supply air temperature $\left({ }^{\circ} \mathrm{C}\right), T_{\text {food }}$ is the food temperature $\left({ }^{\circ} \mathrm{C}\right)$, and $T_{\text {case }}$ is the refrigerator case temperature $\left({ }^{\circ} \mathrm{C}\right)$.

$Q_{A C}$ is the total cooling capacity (W) of the refrigeration case. Unknown factors for $Q_{A C}$ include sensible heat ratio with a typical range of 0.6 to 0.8 and the installed inefficiencies such as long refrigerant lines, low airflow, dirty coils, improper refrigerant charge, etc., which are estimated at $10 \%-20 \%$. Therefore, $C 1$ is introduced to adjust $Q_{A C}$ for unknown factors.

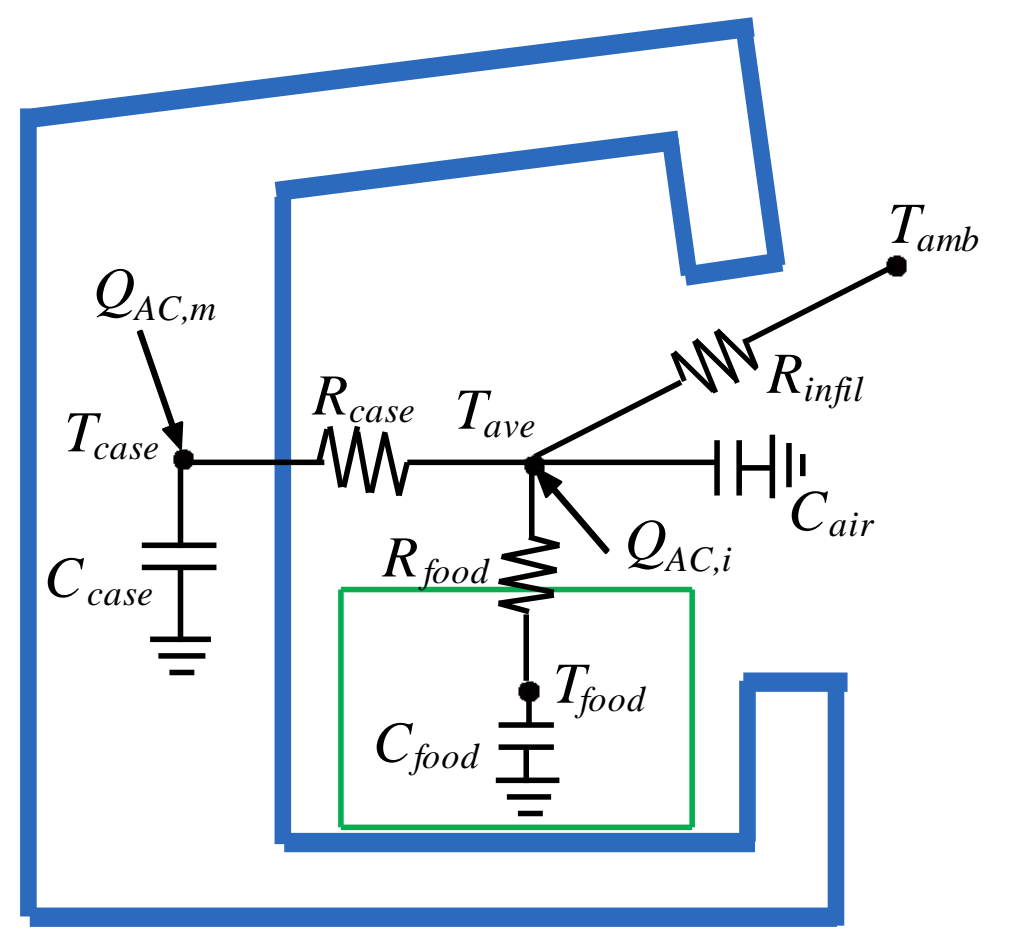

Figure 30: Schematic of simplified open-case $\mathrm{CO}_{2}$ supermarket refrigerator model (3R3C).

Portions of $C 1 Q_{A C}$ (i.e., $C 1 Q_{A C, i}$ ) are transmitted to air in case directly by convection, and the rest (i.e., $\left.C 1 Q_{A C, m}\right)$ is absorbed by the refrigerator case where the subscripts $i$ and $m$ indicate the air in case and internal mass, respectively. They are calculated by Equations 35 and 36:

$$
\begin{gathered}
C 1 Q_{A C, i}=S p 1 * C 1 Q_{A C}, \\
C 1 Q_{A C, m}=(1-S p 1) * C 1 Q_{A C},
\end{gathered}
$$

where, $S p 1$ is the convection fraction for $Q_{A C}$ and is assumed to be unknown and needing to be identified by searching algorithm. 
The searching process for optimal values of the undetermined parameters in this model is a nonlinear optimization process. Given a set of parameters, the gray-box model can predict both the $T_{\text {ave }}$ and $T_{\text {food }}$ profile. An objective function is used to evaluate the fitness between the predicted results and the measured data collected from the reference building during the training period. The objective function $J$ of such optimization is to minimize the integrated RMSEs of both $T_{\text {ave }}$ and $T_{\text {food }}$, as defined in Equation 37.

$$
J\left(R_{\text {case }}, R_{\text {food }}, R_{\text {infil }}, C_{\text {air }}, C_{\text {food }}, C_{\text {case },}, C_{1}, S p\right)=\sqrt{\frac{\sum_{k=1}^{N}\left(T_{\text {ave }, a c t}-T_{\text {ave }, \text { simu }}\right)^{2}}{N-1}}+\sqrt{\frac{\sum_{k=1}^{N}\left(T_{\text {food }, \text { act }}-T_{\text {food,simu })^{2}}\right.}{N-1}},
$$

where $T_{\text {ave,act }}$ and $T_{\text {food,act }}$ are the measured case air and food temperatures. $T_{\text {ave,simu }}$ and $T_{\text {food,simu }}$ are the result from the model. The parameters are identified by the PSO method. PSO is a computational method that optimizes a problem by iteratively trying to improve a candidate solution with regard to a given measure of quality. It solves a problem by having a population of candidate solutions and moving these particles around in the search-space according to simple mathematical formulae over the particle's position and velocity.

\subsubsection{Simplified Compressor Model for Medium Temperature Refrigeration Cycle}

We modeled all three medium temperature compressors (i.e., two fixed-speed compressors and one variable-speed compressor.

Solving classic formula can provide the cooling capacity for the case thermal model and the power consumption for control optimization. However, it is not practical to integrate the entire refrigeration system models into the display case thermal model and control optimization. Thus, further simplification is necessary for predicting the cooling load and power usage. According to the data provided by the compressor manufacturer, a simplified compressor model was developed using Equations 38 and 39:

$$
\begin{gathered}
W_{M T}=C F_{M T} \times\left(b_{0}+b_{1} S M T+b_{2} S M T^{2}+b_{3} S D T+b_{4} S D T^{2}+b_{5} S M T \times S D T\right), \\
Q_{M T}=C F_{M T} \times\left(b_{6}+b_{7} S M T+b_{8} S M T^{2}+b_{9} S D T+b_{10} S D T^{2}+b_{11} S M T \times S D T\right),
\end{gathered}
$$

where, $S M T$ is the refrigerant saturation temperature of the middle stage, which is equivalent to the saturation suction temperature for the medium-temperature compressors. SDT is the refrigerant saturation discharge temperature for the medium-temperature compressor. $b_{0}, b_{1} \ldots b_{11}$ are parameters that can be calibrated through compressor manufacturer data.

$C F_{M T}$ is the correction factor for partial load operation with compressor variable frequency drive, which is applicable for the variable-speed medium-temperature compressor $\left(4 \mathrm{MTC} \_10 \mathrm{~K} \_\mathrm{V}\right)$ only. The $\mathrm{CF}_{M T}$ can be calculated using Equation 40:

$$
C F_{M T}=\left(k_{0}+k_{1} S M T+k_{2} S D T+k_{3} S M T \times S D T\right) .
$$

The SMT is controlled by the refrigeration system to maintain a saturation temperature low enough to keep the coldest medium temperature case in the loop cool. For our system, the setting is $\sim 380$ psig or a $S M T$ of $13.4^{\circ} \mathrm{F}$.

The SDT is dependent on the outdoor temperature because of the condenser rejecting heat to the outside air. Based on test data, the relationship shown in Figure 31 was developed to correlate SDT to outdoor temperature and was converted to Equations 41-43. 


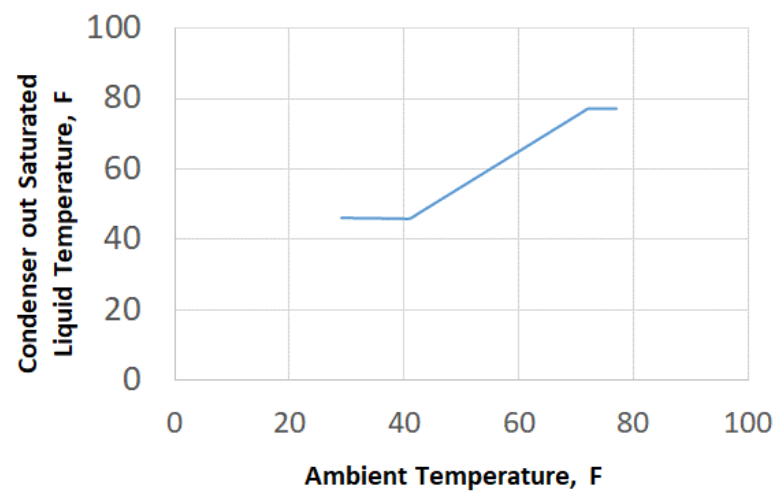

Figure 31: Relationship between outdoor dry bulb temperature $\left(\boldsymbol{T}_{\text {out }}\right)$ and condenser out saturated liquid temperature (SDT).

$$
\begin{gathered}
T_{\text {out }}<41^{\circ} \mathrm{F}: S D T=46.791-0.0214^{*} T_{\text {out }} . \\
41^{\circ} \mathrm{F}<=T_{\text {out }}<72^{\circ} \mathrm{F}: S D T=T_{\text {out }}\left({ }^{\circ} \mathrm{F}\right)+5 . \\
T_{\text {out }}>=72^{\circ} \mathrm{F}: \quad S D T=77^{\circ} \mathrm{F} .
\end{gathered}
$$

\subsubsection{Training and Testing Results of the Developed Models}

\subsubsection{Training and testing results of the open-case refrigerator display case thermal model}

The refrigeration case (RC) model was tested and validated with data collected from the reference refrigeration system in two different consecutive time periods with various operation conditions (e.g., different schedules of supply air temperature set points). The data collected from March 1, 2019, to March 1, 2019 (two consecutive days), are used for the training section, and the data collected from March 3, 2019, to March 5, 2019 (three consecutive days), are used for validation. The time steps in both training and validation data sets are 5 minutes.

The training and testing results are shown in Figure 32 and Figure 33. $T_{\text {ave act }}$ and $T_{\text {food,act }}$ are the measured case supply air and food temperatures, respectively. $T_{a v e, R C}$ and $T_{\text {food,RC }}$ are the case supply air and food temperatures from the model for a 24-hour prediction horizon. The resulting parameters identified by PSO are $R_{\text {infil }}=0.00575 \mathrm{~K} / \mathrm{W}, R_{\text {food }}=0.003915 \mathrm{~K} / \mathrm{W}, R_{\text {case }}=0.03647 \mathrm{~K} / \mathrm{W}, C_{\text {air }}=75,964 \mathrm{~J} / \mathrm{K}, C_{\text {food }}=$ $3,245,969 \mathrm{~J} / \mathrm{K}, C_{\text {case }}=19,109,514 \mathrm{~J} / \mathrm{K}, C l=0.1792$, and $S p l=0.6862$.

To quantify the deviations of the predicted data from the measured data in both training session and testing sessions, RMSE indices are used to evaluate the deviations, as listed in Table 7.

It can be found that the developed RC model has satisfactory performance in prediction of case supply air and food temperatures. 
Table 7: Accuracy indices of the RC model

\begin{tabular}{cccc}
\hline Time & Training/testing & $\boldsymbol{T}_{\text {ave }}$ & RMSE \\
& & 1.19 & $\boldsymbol{T}_{\text {food }}$ \\
\hline 3/1/2019 to 3/2/2019 & Training & 1.48 & 0.21 \\
3/3/2019 to 3/5/2019 & Testing & 0.40 \\
\hline
\end{tabular}

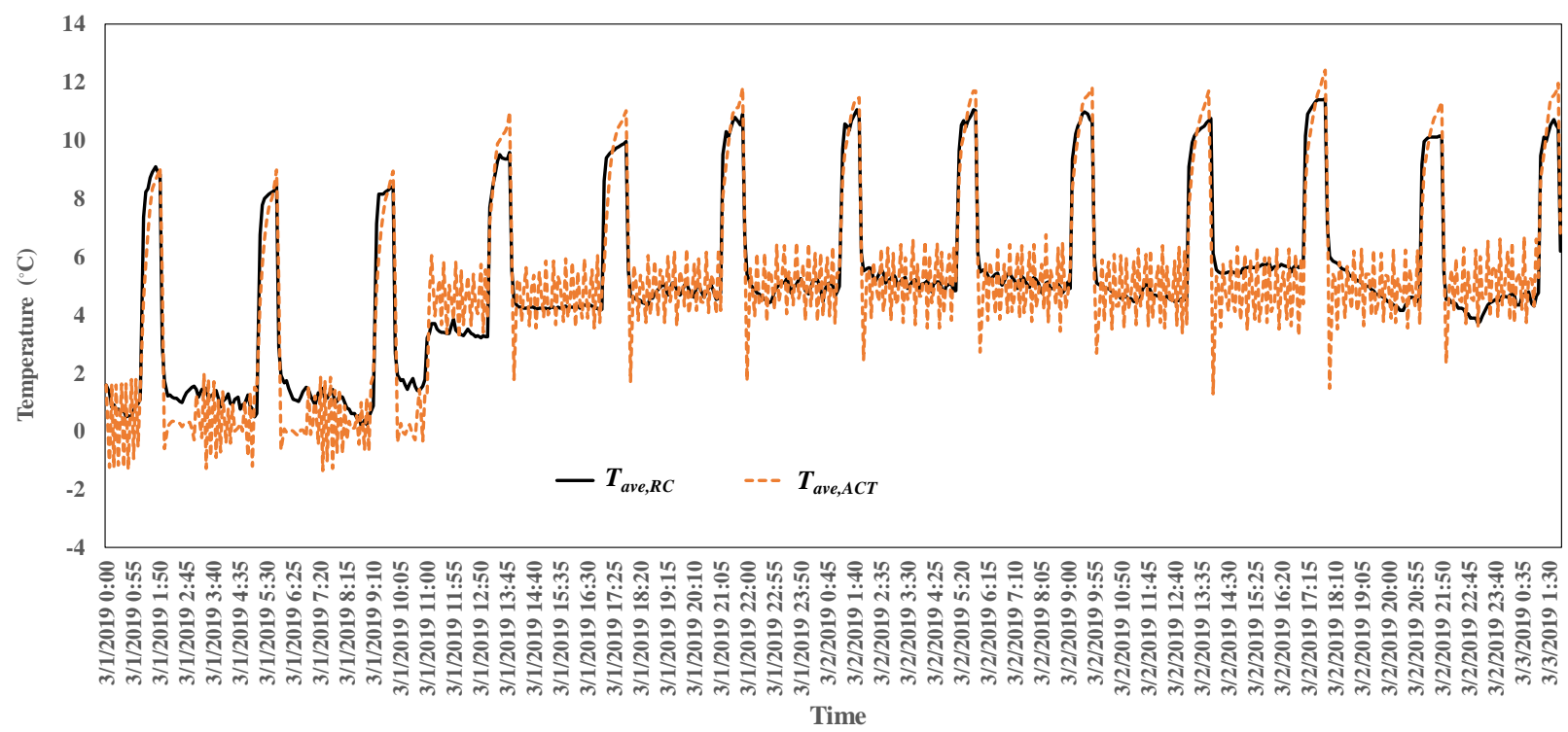

(a) Case supply air temperature comparison

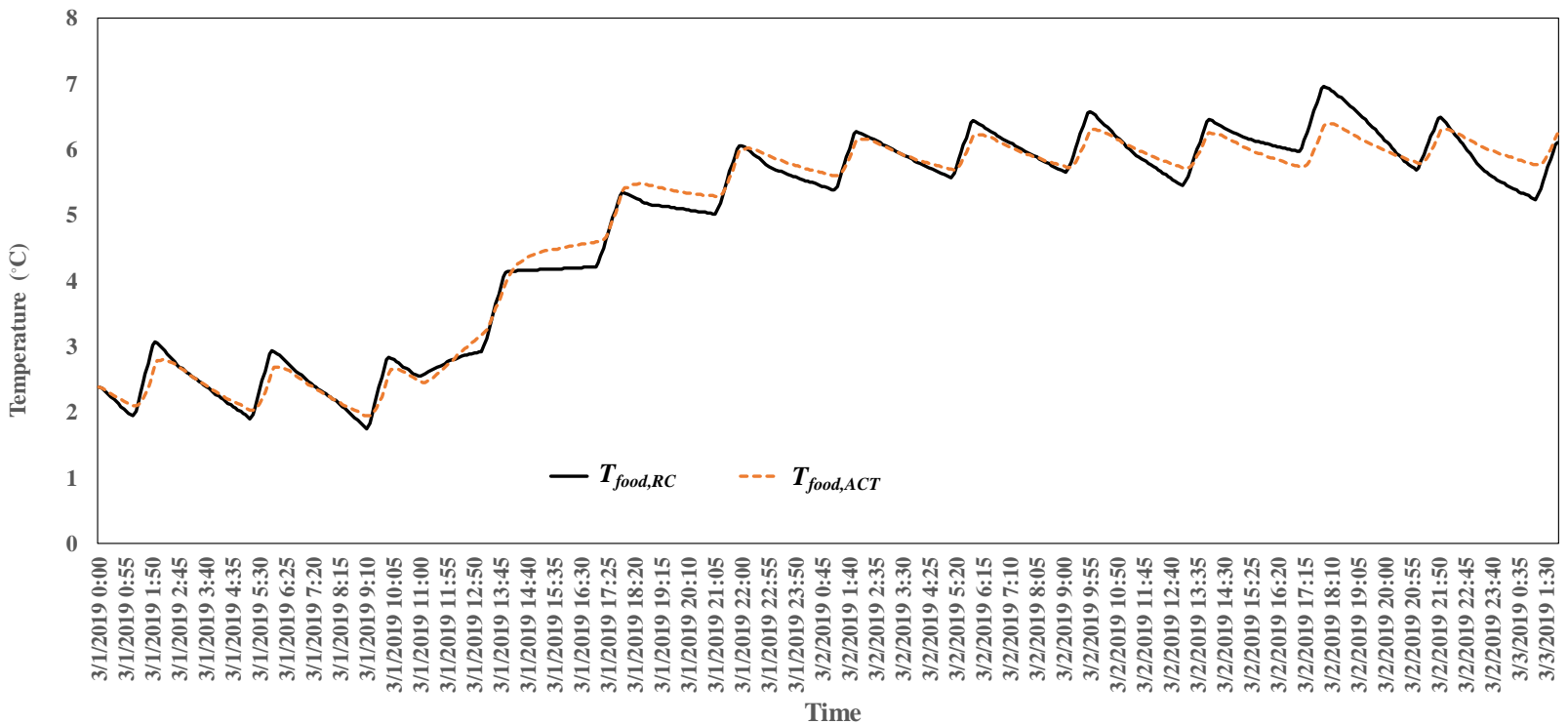

(b) Food temperature comparison

Figure 32: Open-case refrigerator display case model training results from March 1-2, 2019. 


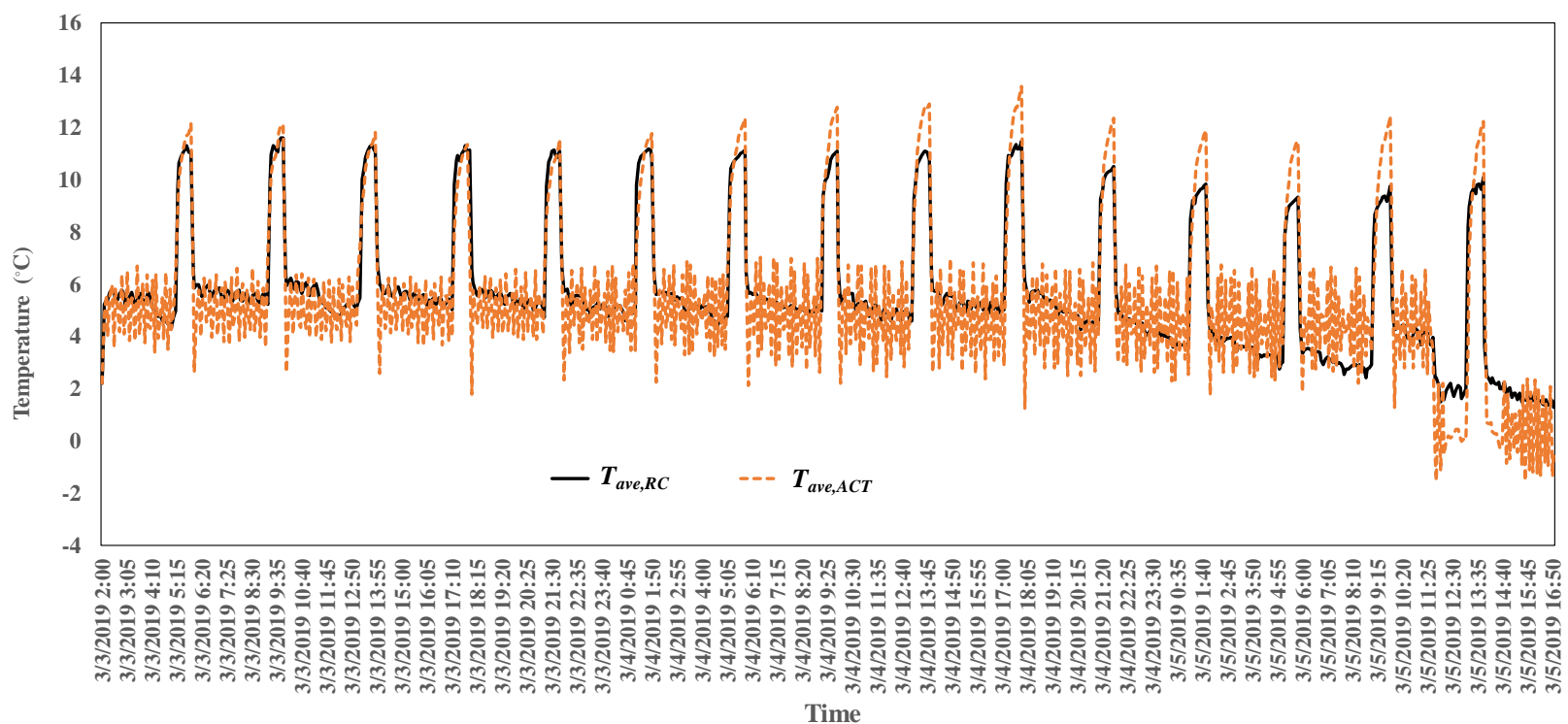

(a) Case supply air temperature comparison

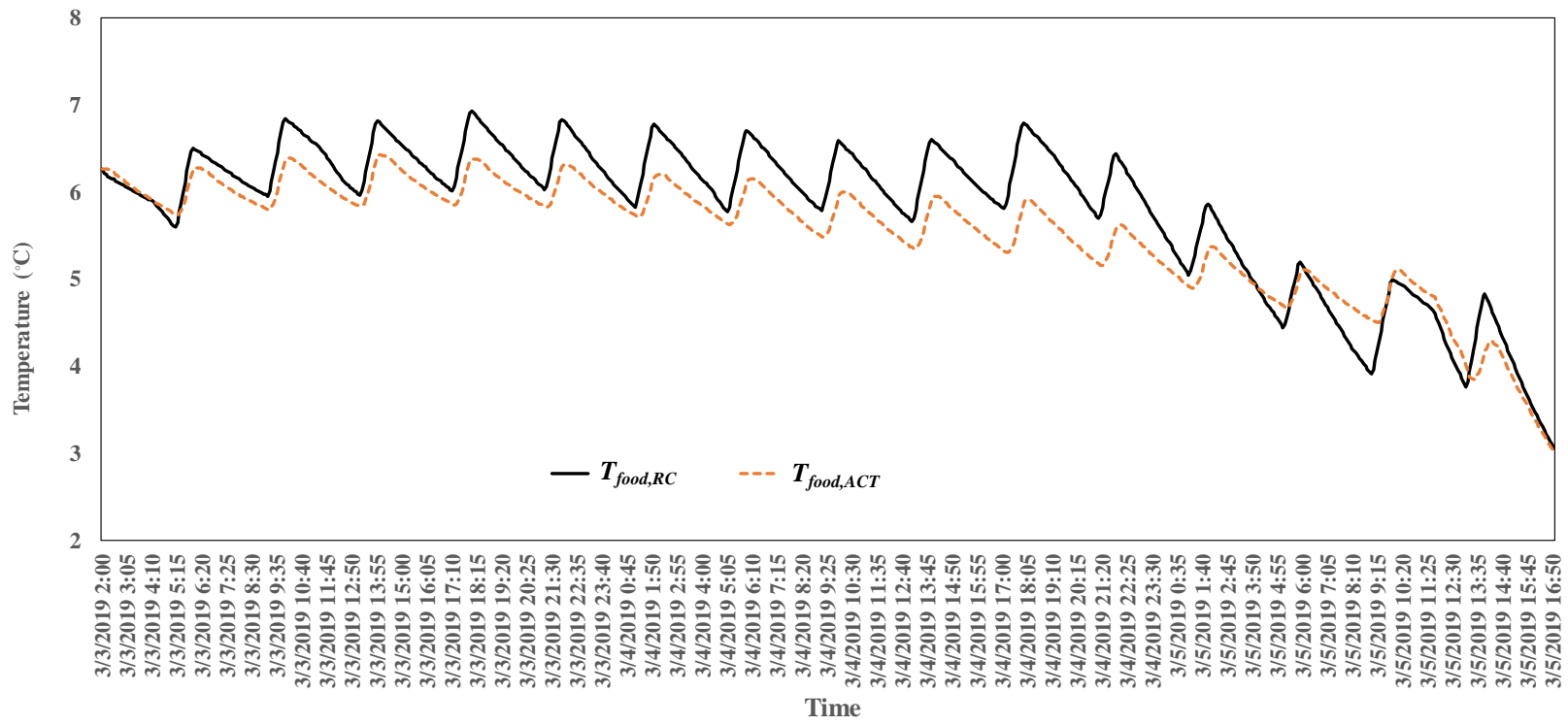

(b) Food temperature comparison

Figure 33: Open-case refrigerator display case model testing results from Mar 3-5, 2019.

\subsubsection{Training and testing results of the simplified compressor model}

The reduced model for cooling load and power consumption prediction is calibrated with the manufacturer's data. All parameters are identified and listed in Table 7. 
Table 7. Calibrated parameters for simplified compressor model

\begin{tabular}{|c|c|c|c|}
\hline Parameters & 4MTC-7K & 4MTC-10K & 4MTC-10K_V \\
\hline $\mathrm{b}_{0}$ & 3743.692211 & 3677.089459 & 3677.089459 \\
\hline $\mathrm{b}_{1}$ & -113.1469513 & -115.2126198 & -115.2126198 \\
\hline $\mathrm{b}_{2}$ & -0.949968838 & -0.909689722 & -0.909689722 \\
\hline $\mathrm{b}_{3}$ & 5.866269538 & 5.977244154 & 5.977244154 \\
\hline $\mathrm{b}_{4}$ & 0.426104769 & 0.478541268 & 0.478541268 \\
\hline $\mathrm{b}_{5}$ & 1.741848346 & 1.707071315 & 1.707071315 \\
\hline $\mathrm{b}_{6}$ & 31930.52927 & 38545.43758 & 38545.43758 \\
\hline $\mathrm{b}_{7}$ & 802.6804349 & 895.2242371 & 895.2242371 \\
\hline $\mathrm{b}_{8}$ & 2.930720513 & 3.439209561 & 3.439209561 \\
\hline $\mathrm{b}_{9}$ & -201.986731 & -427.6732271 & -427.6732271 \\
\hline $\mathrm{b}_{10}$ & -0.343492271 & 1.427300647 & 1.427300647 \\
\hline $\mathrm{b}_{11}$ & -5.958668834 & -7.221110318 & -7.221110318 \\
\hline $\mathrm{k}_{0}$ & - & - & 0.861088 \\
\hline $\mathrm{k}_{1}$ & - & - & -0.00307 \\
\hline $\mathrm{k}_{2}$ & - & - & $-4.2 \mathrm{E}-05$ \\
\hline $\mathrm{k}_{3}$ & - & - & $3.90 \mathrm{E}-06$ \\
\hline
\end{tabular}

Figure 34 and Figure 35 indicate that the model calibration has a high level of accuracy with $\pm 2 \%$ error relative to the manufacturer's data for all compressors.
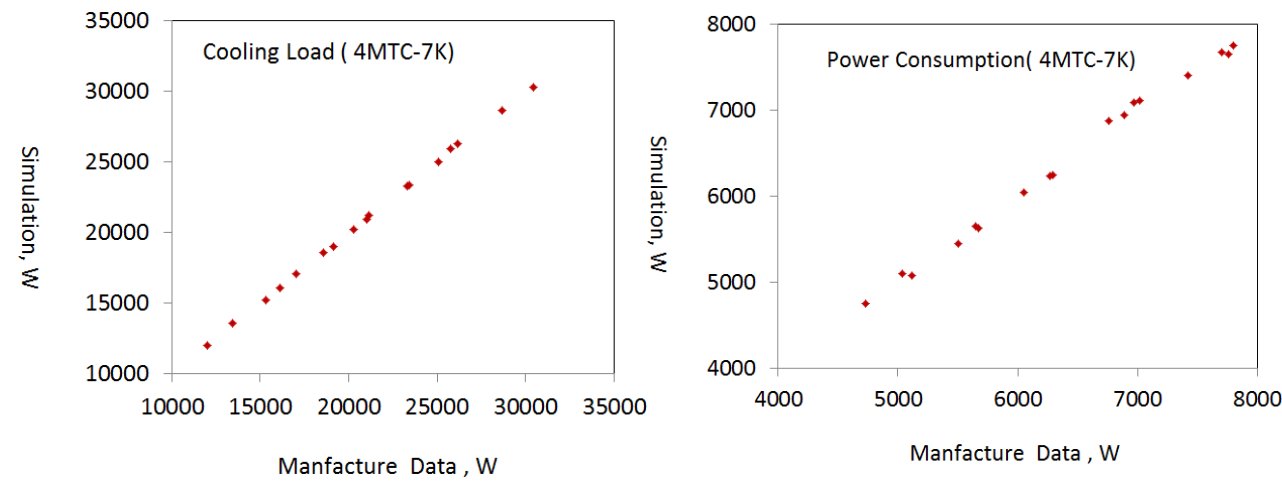

Figure 34: Model calibration of compressor 4MTC-7K.
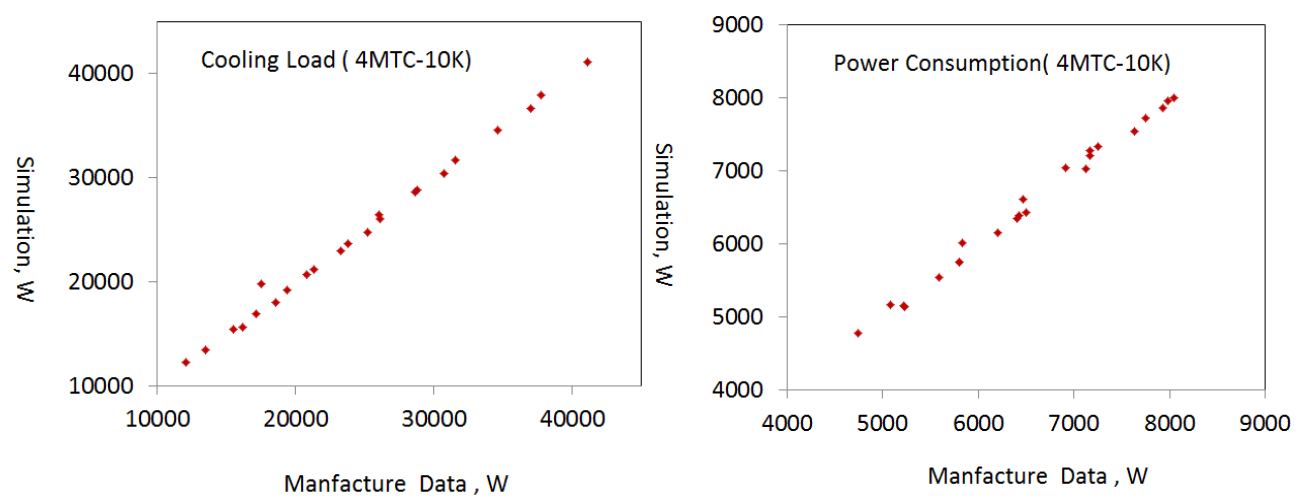

Figure 35: Model calibration of compressor 4MTC-10K. 
Two data sets are used to show the prediction accuracy of total power consumption. The first data set was collected from 5:00 p.m. on February 6, 2019, to 12:00 p.m. on February 7, 2019. The second data set was collected from 5:00 p.m. on February 7, 2019, to 12:00 p.m. on February 8, 2019. The power prediction of total power consumption is compared with the testing data. The results are shown in Figure 36 and Figure 37 with the model inputs as $S M T$ and $S D T$.

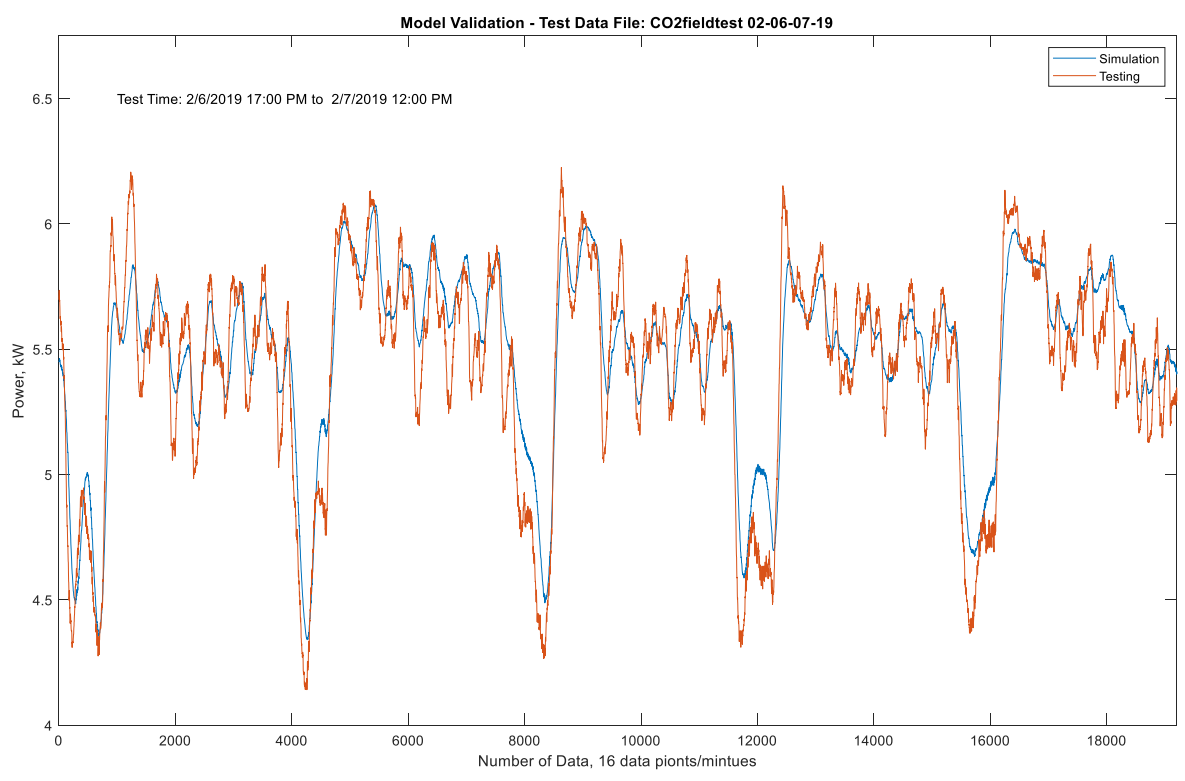

Figure 36: Medium temperature compressor power consumption comparison of test data 1.

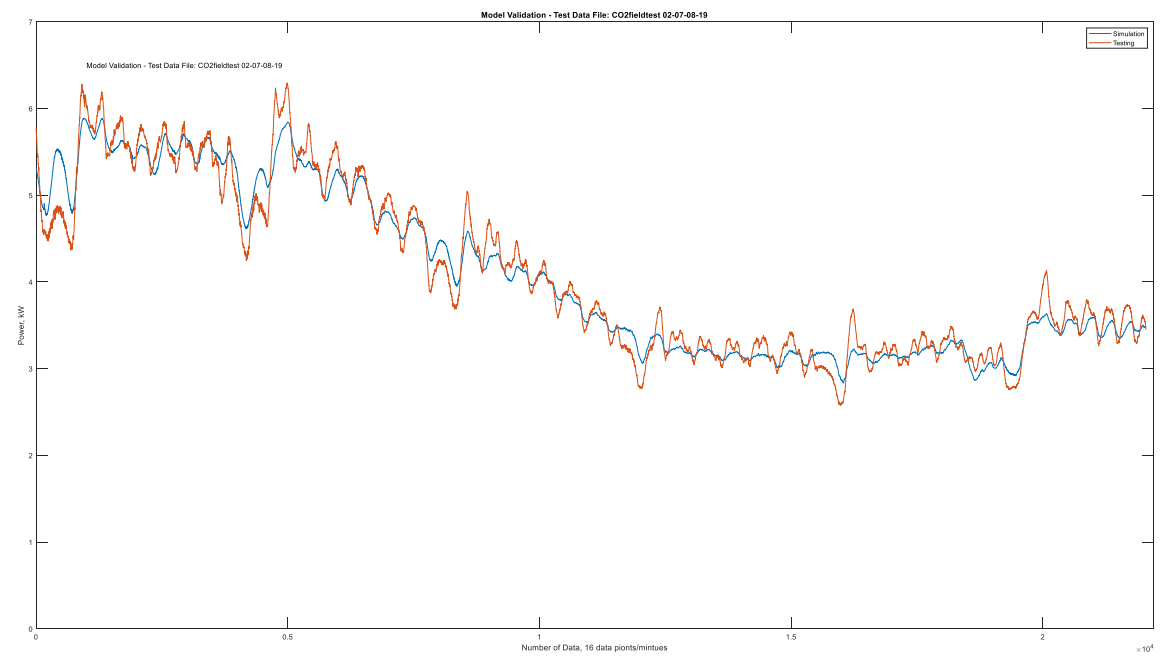

Figure 37: Medium temperature compressor power consumption comparison of test data 2. 


\subsubsection{Hourly Load Flexibility Through Priority-Based Control}

Similar to that flexibility analysis of water heaters in Section 4.2, the flexibility of supermarket refrigeration systems is analyzed for hour-long periods. Simulations for all hours of two consecutive days (i.e., 48 hours) were run. Both load-shed and load-up scenarios are also assumed for supermarket refrigeration systems. The load-shed and load-up results are analyzed using two metrics; the peak power change and the energy flexibility potential. The peak power flexibility is the difference between the 5minute peak power use during the load-shed/load-up hour of the baseline and the PBC cases. The energy reduction potential is the difference between the energy use during the load-shed/load-up hour for the baseline and PBC cases.

\subsubsection{System and operation assumptions}

For comprehensive quantification of load flexibility of multiple supermarket refrigeration systems, four sets of systems are considered. Each set includes the same compressors as introduced previously (i.e., two fixed-speed compressors and one variable-speed compressor). The total rated cooling capacity is $34,000 \mathrm{~W}(11,100+11,400+11,300 \mathrm{~W})$. Each compressor set is modeled with 12 display cases since the rated cooling capacity of each one is $2,810 \mathrm{~W}$. One important assumption in this research is that the cooling capacity of each display case is assumed to be fixed.

The power of four different refrigeration systems is generated by randomizing the initial statuses of the 48 connected display cases, that is, defrost status, recovery status, air-in-case temperature, food temperature, case temperature, and accumulated running time, as shown in Figure 38. In addition, to embody the differences among these refrigeration systems, the parameter $C_{\text {food }}$ in the open-case refrigerator display case thermal model is randomly varied from 0.8 times to 1.2 times the trained value (i.e., 3,245,969 J/K). This adds variation to the refrigerators to provide more realistic and varied responses.

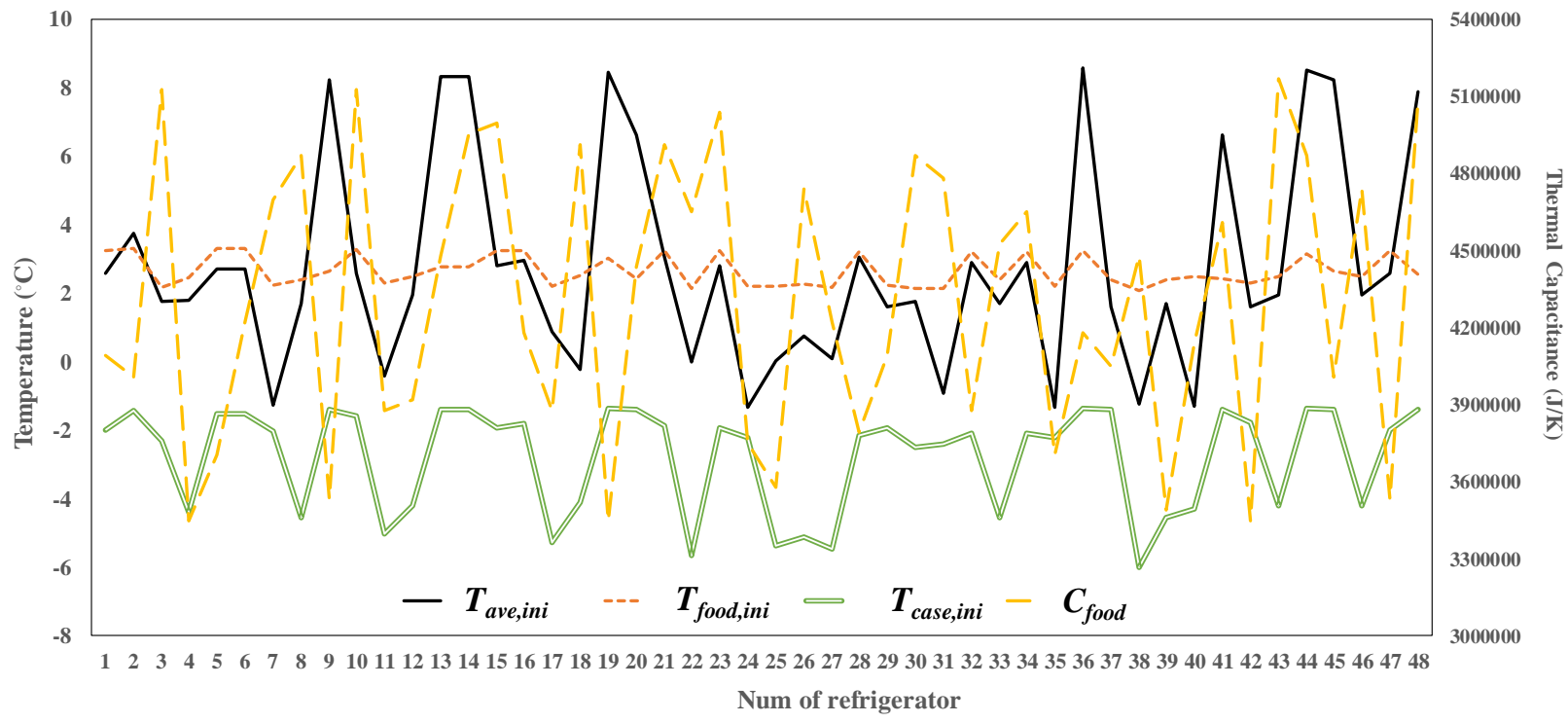

Figure 38: Initial settings of $T_{\text {ave }}, T_{\text {food, }}$ and $T_{\text {case }}$ for 50 refrigerators.

Typical medium temperature display cases go through six defrost cycles per day in which refrigeration to the case is shut down for $\sim 40$ minutes. Therefore, each single display case needs to be shut down to defrost after it accumulates 3 hours and 20 minutes ( 40 time steps) of run time. The defrost duration is fixed for each refrigerator (i.e., 40 minutes [ 8 time steps]). The defrost duration is followed by a recovery period. Recovery means each display case must be on for at least 60 minutes (12 time steps) after the 40- 
minute defrost process. During this 60-minute period, the display case cannot be interrupted and shut down.

The same initial settings are used for all simulations and were run for two consecutive days (i.e., March $1-2,2019$.

The on/off control of the compressors in each set is governed by the following rules:

1. Calculate the total running number of display cases (num) at i timestep.

2. Calculate the required total cooling capacity based on rated capacity of each display case: 2,810*num.

3. If $2,810^{*}$ num $<=11100 \mathrm{~W}: 4 \mathrm{MTC}-10 \mathrm{~K} \_\mathrm{V}: 0 \mathrm{~N} ;$ 4MTC-10K: OFF; 4MTC-7K: OFF;

If $11100 \mathrm{~W}<2,810^{*}$ num <= 22500W: 4MTC-10K_V:ON; 4MTC-10K: ON; 4MTC-7K: OFF; If 2,810*num > 22500W: 4MTC-10K_V:ON; 4MTC-10K: ON; 4MTC-7K: ON.

\subsubsection{Baseline operation}

For the baseline simulation, all of the cases are initialized with their randomized statuses (i.e., defrost status, recovery status, air temperature, food temperature, case temperature, and accumulated run time). When each refrigeration case accumulates 3 hours and 20 minutes of run time, it will be shut down for defrosting for a period of 40 minutes. After the defrost, the unit turns back on and runs for another 3 hours and 20 minutes, repeating the cycle.

The aggregated power from the four sets of compressors is shown in Figure 39. The power fluctuates between 38 to $57 \mathrm{~kW}$. As seen in Figure 39, $T_{a m b}$ (i.e., the indoor temperature surrounding the display case) is relatively stable and fluctuates between $17.2^{\circ} \mathrm{C}$ to $21.5^{\circ} \mathrm{C} . T_{\text {out }}$ (i.e., the outdoor dry bulb air temperature) has a relatively large fluctuation ranging from $5.1{ }^{\circ} \mathrm{C}$ to $14.5^{\circ} \mathrm{C}$. The trend/magnitude of the total power consumption of all compressors follows the shape of $T_{\text {out }}$ since power consumption of each compressor is dependent on outdoor dry bulb air temperature. This is why the flexibility of the supermarket refrigeration system is analyzed at all hours of the day. 


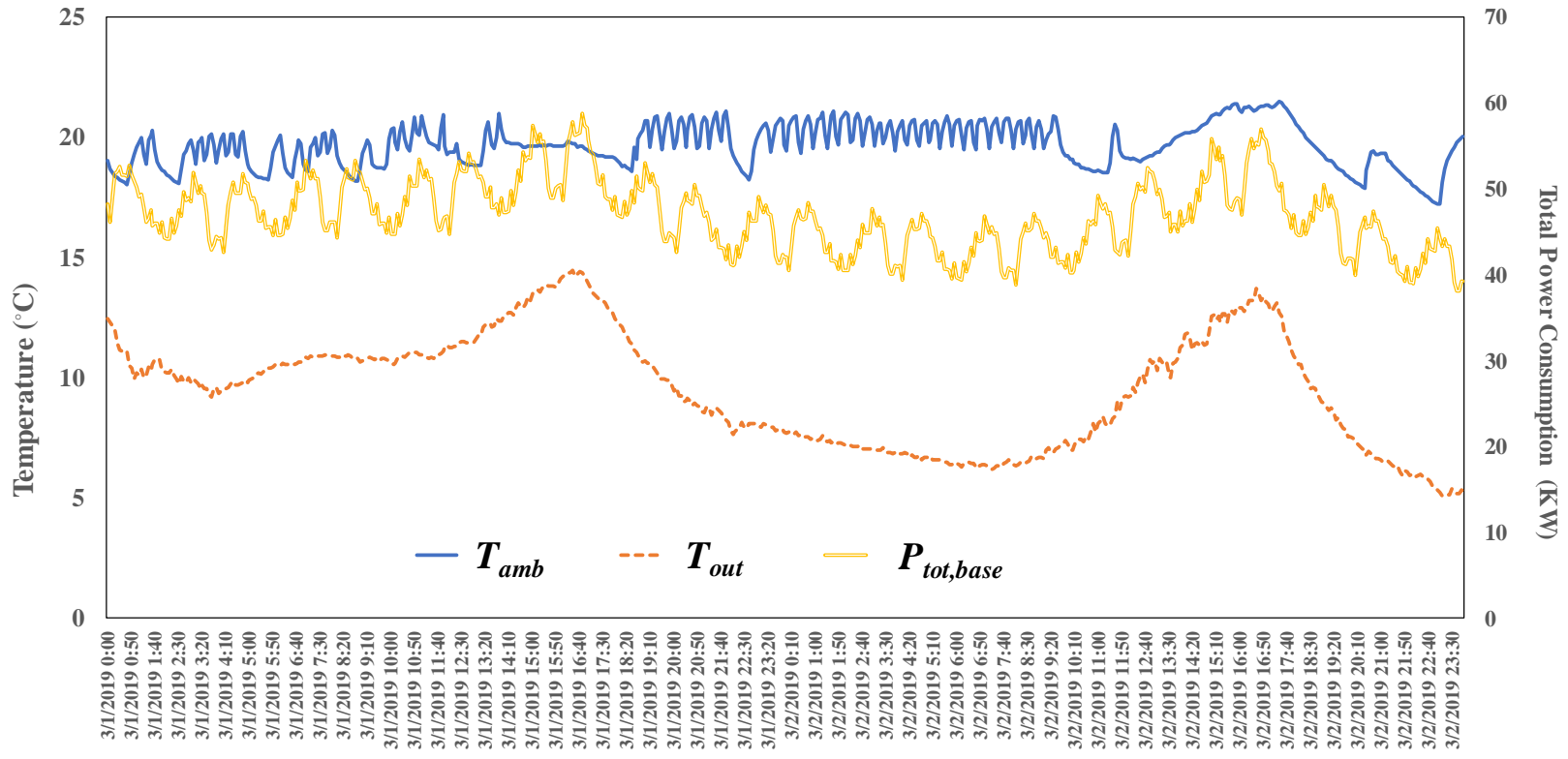

TIME

Figure 39: Aggregate power consumption for 48 supermarket refrigeration systems under baseline operation.

Figure 40 shows the total number of display cases running and the average display case running status in each time step. The running status of each display case is zero when it is in defrost period; otherwise the running status ranges from 1 to 40 (40 means the maximum accumulated time steps before the display case defrosts) when it is not in defrost period.

Obviously, both the total running number of display cases and the average display case running status follow a pattern with a 4-hour frequency. This is because the initial status of each display case is randomly initialized and 4 hours is the operation cycle for defrosting (i.e., 3 hours and 20 minutes for total running time and 40 minutes for the defrost period).

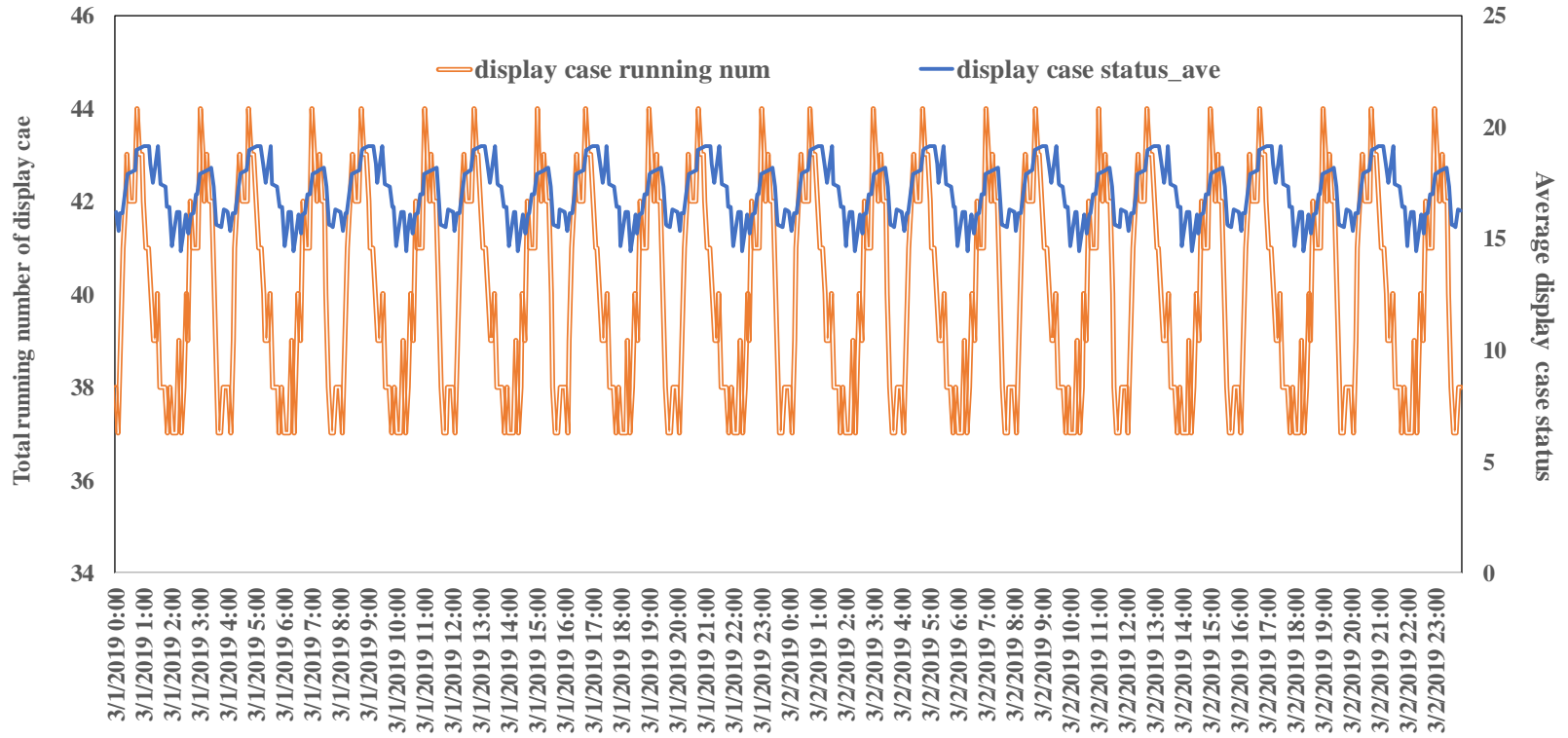

TIME

Figure 40: Aggregate power consumption for 48 supermarket refrigeration systems under baseline operation. 


\subsubsection{PBC implementation-hourly load shed}

PBC was also implemented for each hour of the day. The control is to turn off the display cases one by one until the total compressor power consumption is not higher than a preset power limit during each load-shed hour. If the total power consumption in any time step is higher than the power limit, the trial is unsuccessful. Following an unsuccessful trial, the power limit will be increased until the total power consumption in each time step is not higher than the target.

A list is formed to include the display cases that can be turned off during the current time step. The status of each display case in this list obeys such criteria: the accumulated running time is less than 40 time steps and it is neither in the defrost period nor the recovery period. The display cases with lower $T_{\text {ave }}$ take priority of selection in the list since it is safer to turn off the display cases with the lowest $T_{\text {ave }}$ first. (It could be better to take $T_{\text {food }}$ as an indicator to show the status of the food directly, but $T_{\text {food }}$ cannot be measured in a real application.)

\subsubsection{PBC implementation-hourly load-up}

The control for the hourly load-up scenario is to turn on the display cases that are currently defrosting one by one until the total compressor power consumption is not lower than the preset target during each loadup hour. If the total power consumption in any time step of the load-up hour is lower than the power limit, the trial is unsuccessful. Following an unsuccessful trial, the target power will be reduced until the total power consumption in each time step of the hour is not lower than it.

A list is formed to include the display cases that can be turned on during the current time step. Note that one big difference between this scenario and the baseline operation or the load-shed scenario is that the display cases can be started before the original defrost period (duration of 40 minutes) ends. It is assumed that display cases that have already been defrosted for no less than 20 minutes can be turned on. Such curtailment of defrost duration is due to the fact that the default setting/status of each display case is always on under baseline operation and its cooling demand is fixed as introduced previously. Relaxing the defrost period allows the total cooling demand from display cases to be larger than that under baseline operation, and is expected to be reasonable since time-based defrost schedules are often conservative and result in unnecessary defrost cycles.

Therefore, the status of each display case in the list obeys this criteria: the display case is in defrost period, and the accumulated defrost time has already been at least 20 minutes. The display cases with higher $T_{\text {ave }}$ take priority of selection in the list since it is safer to first turn on the display cases with higher $T_{\text {ave. }}$.

\subsubsection{Simulation Results}

Sample plots showing the comparison of results, including total power consumption, supply air temperatures, and food temperatures, under baseline operation and hourly load shed with $\mathrm{PBC}$ for the period from 4:00 a.m. to 5:00 a.m. are shown in Figures 41 through 43.

The power consumption of each compressor set is independent from the others. The defrost periods of some display cases, which are shut down for reducing the overall compressor power consumption under the targeted power limit (i.e., $P_{\text {targeted }}$ ), have to be postponed until after the load-shed period. Therefore, the original schedules of these display cases are changed accordingly. This can cause a more synchronized situation in that after the shed period, the power "valley" could be even lower and the peak could be even higher in the power profile under PBC since the statuses of display cases could be more synchronized, as shown in Figure 41, Figure 42, and Figure 43. 
Sample plots showing the comparison of results, including total power consumption, supply air temperatures, and food temperatures, under baseline operation and hourly load-up with the period from 12:00 p.m. to 1:00 p.m. are shown in Figure 44, Figure 45, and Figure 46. Compared with the changes in the load-shed scenario, the changes of power, $T_{a v e}$ and $T_{\text {food }}$, in the hourly load-up scenario are much less since fewer display cases are defrosting than running at any point in time and only the display cases that have already been defrosting for at least 20 minutes are allowed to be turned on early.

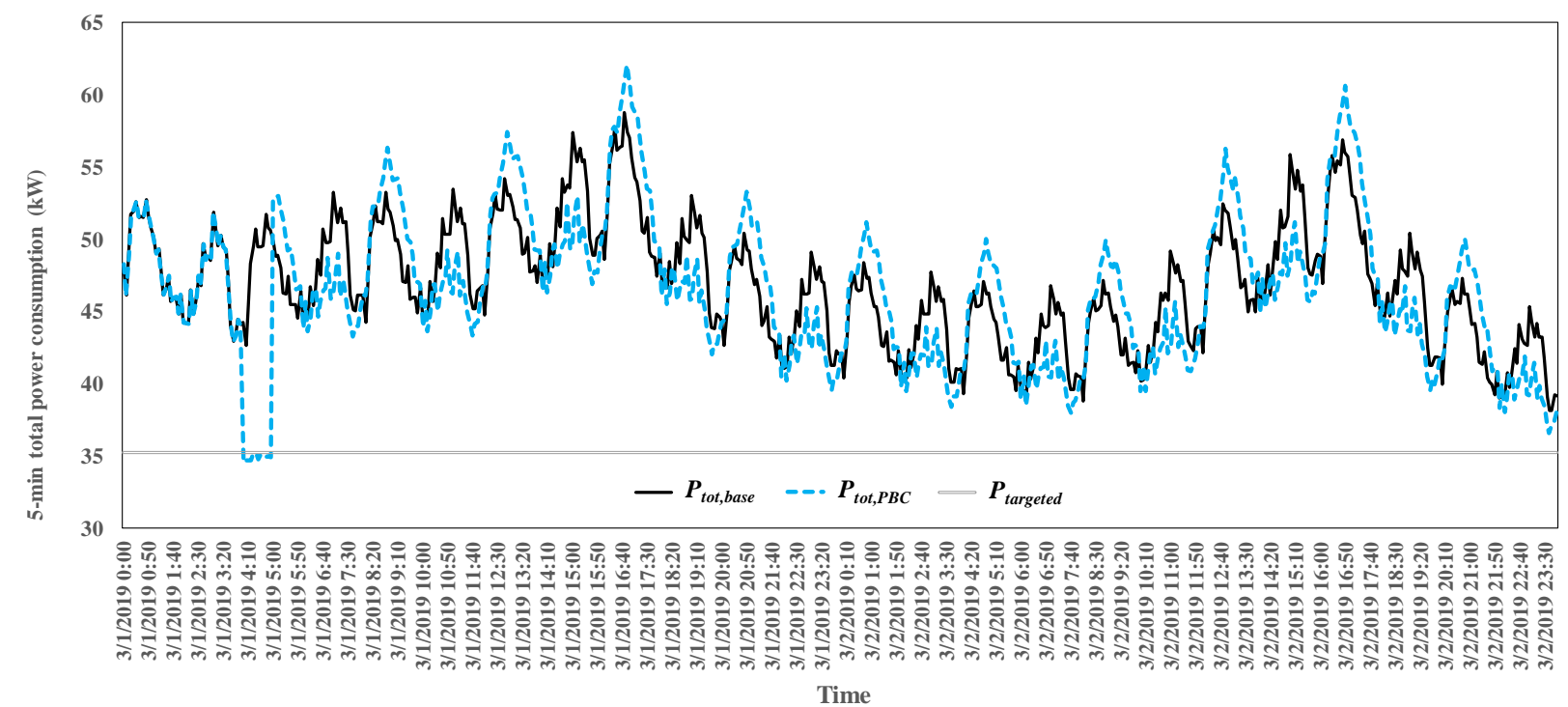

Figure 41: Total power consumption profiles for baseline and PBC with a load-shed period of 4:00 a.m. to 5:00 a.m., March 1, 2019.

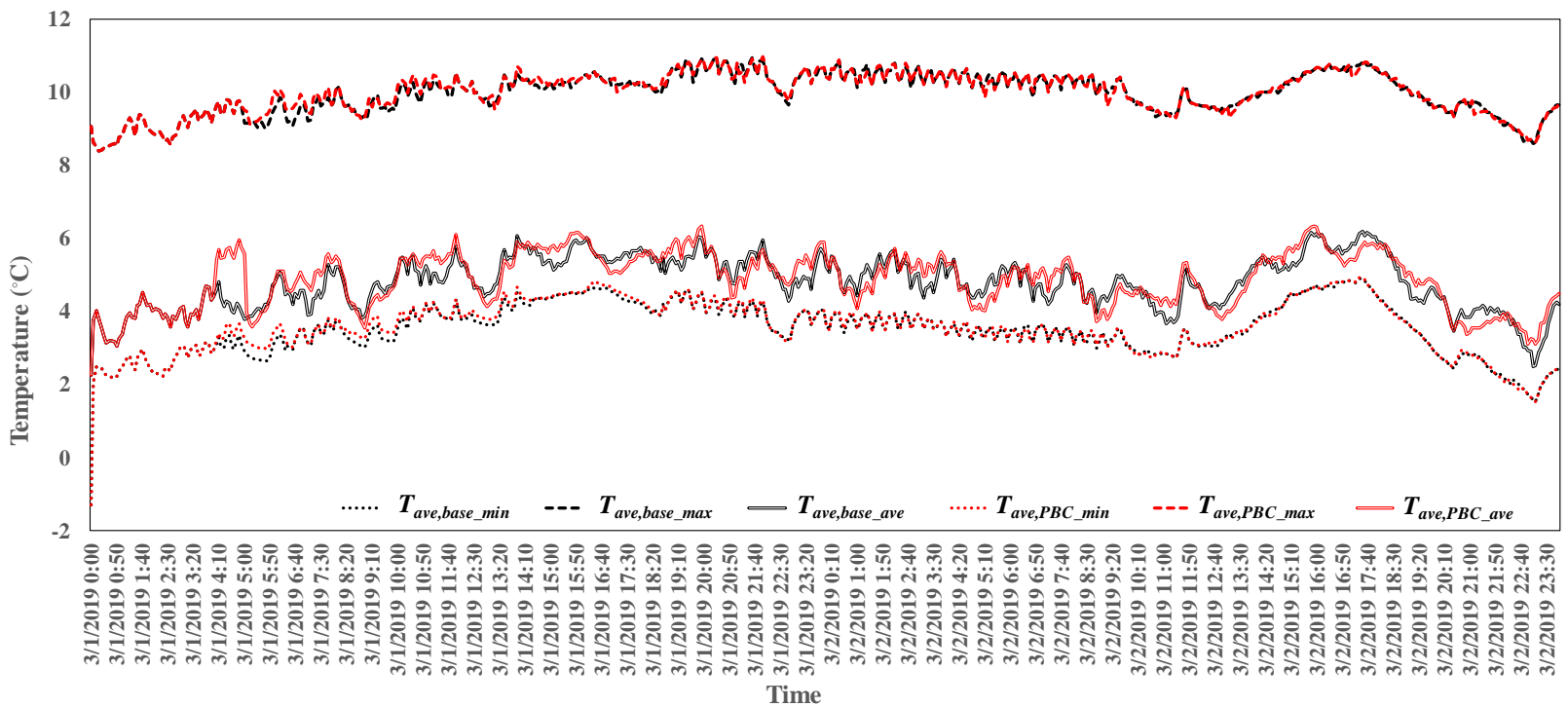

Figure 42: Supply air temperature profiles for baseline and PBC with a load-shed period of 4:00 a.m. to 5:00 a.m., March 1, 2019. 


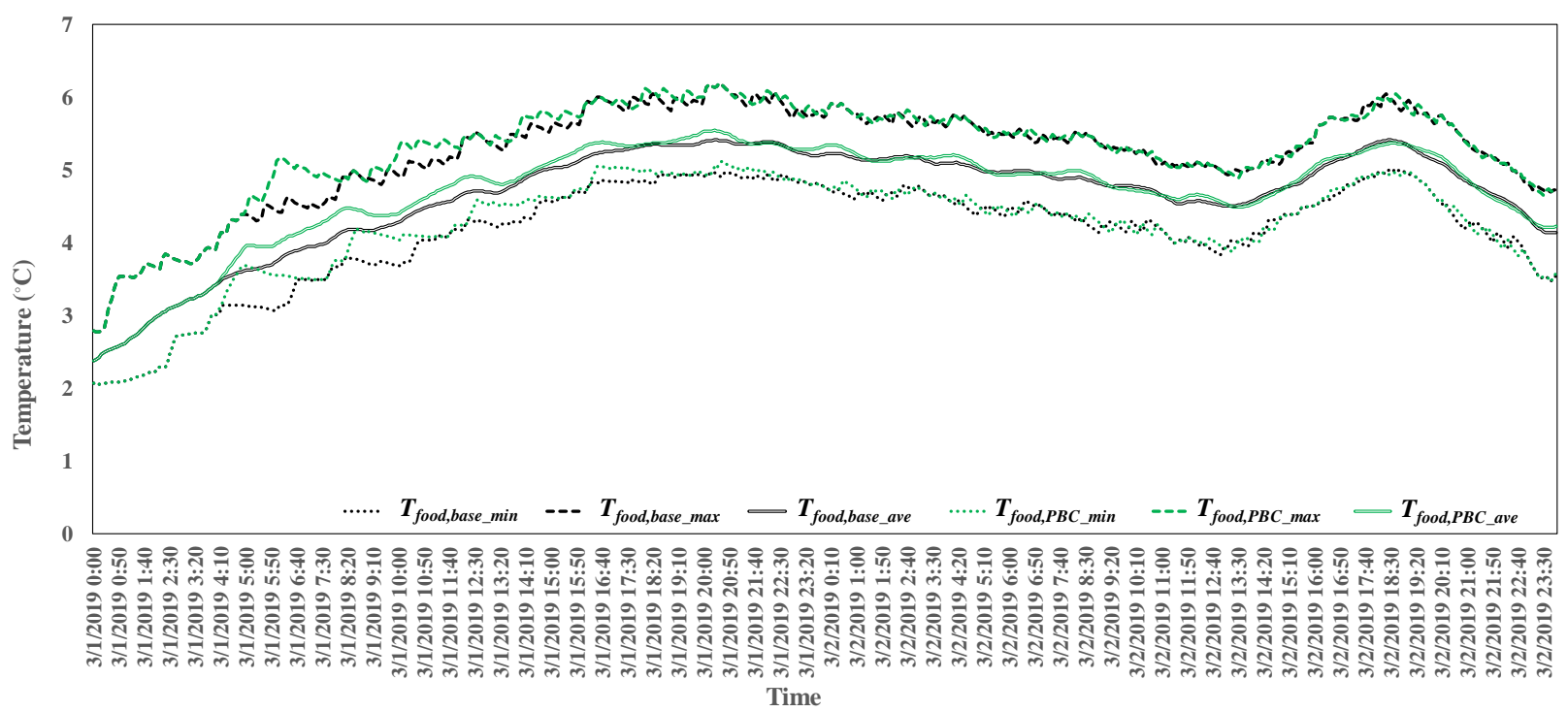

Figure 43: Frozen food temperature profiles for baseline and PBC with a load-shed period of 4:00 a.m. to 5:00 a.m., March 1, 2019.

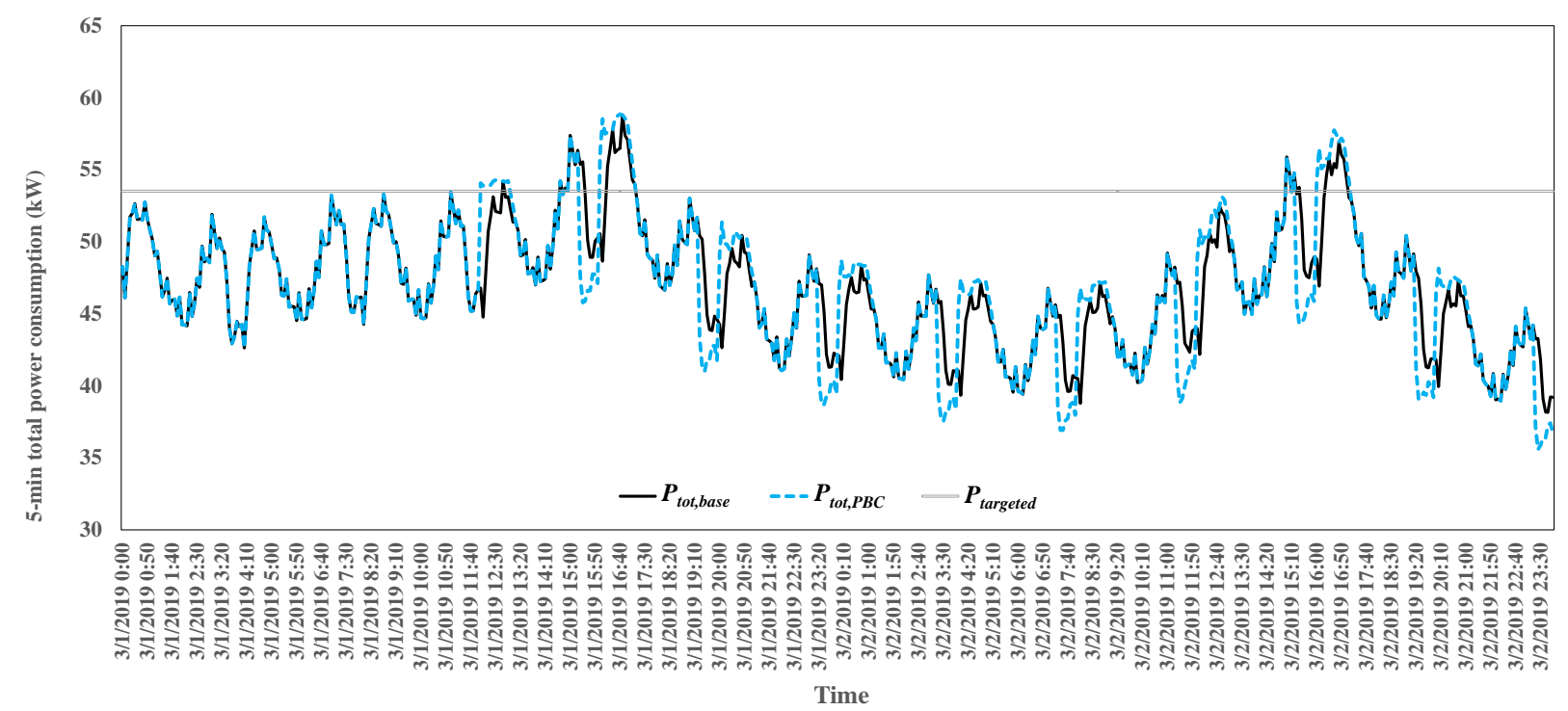

Figure 44: Total power consumption profiles for baseline and PBC with a load-up period of 12:00 p.m. to 1:00 p.m., March 1, 2019. 


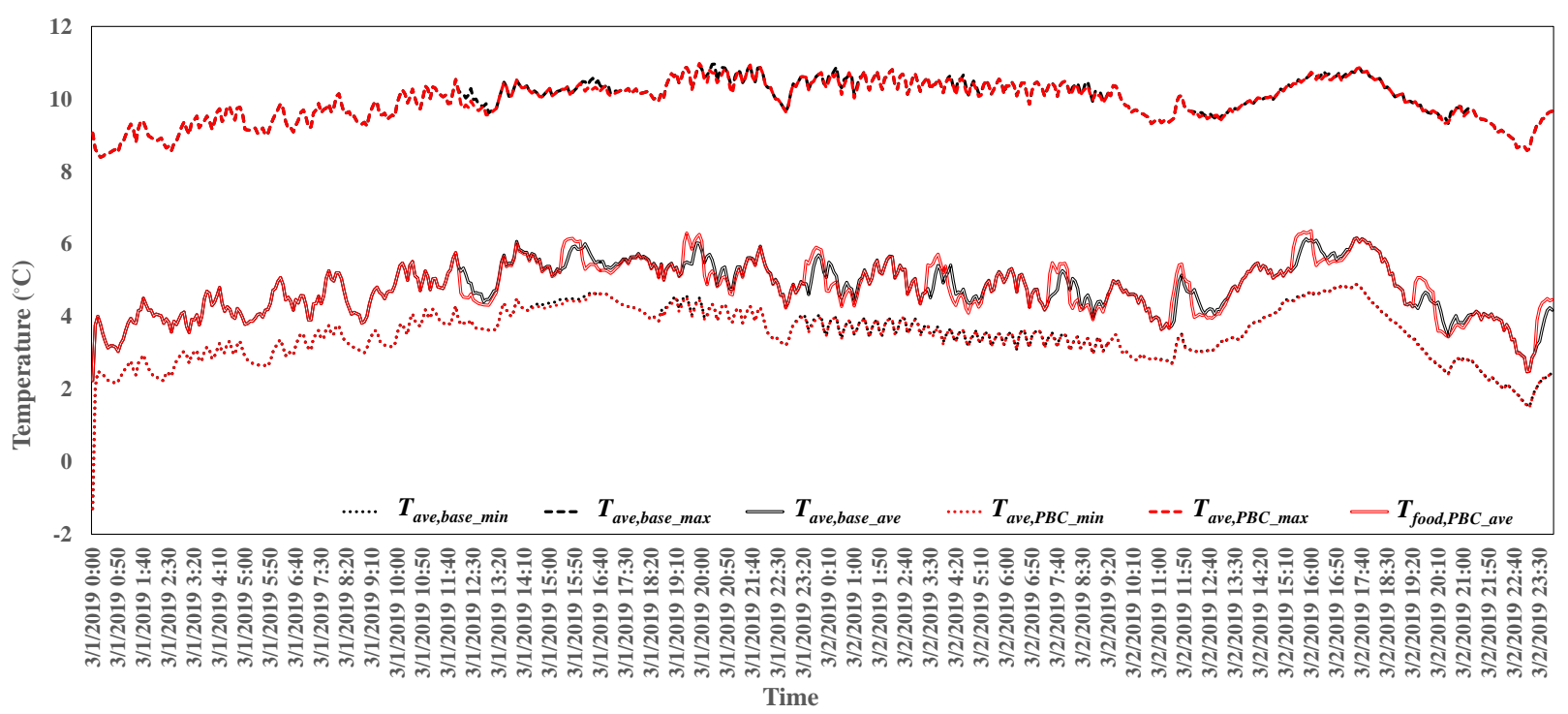

Figure 45: Air-in-case temperature profiles for baseline and PBC with a load-up period of 12:00 p.m. to 1:00 p.m., March 1, 2019.

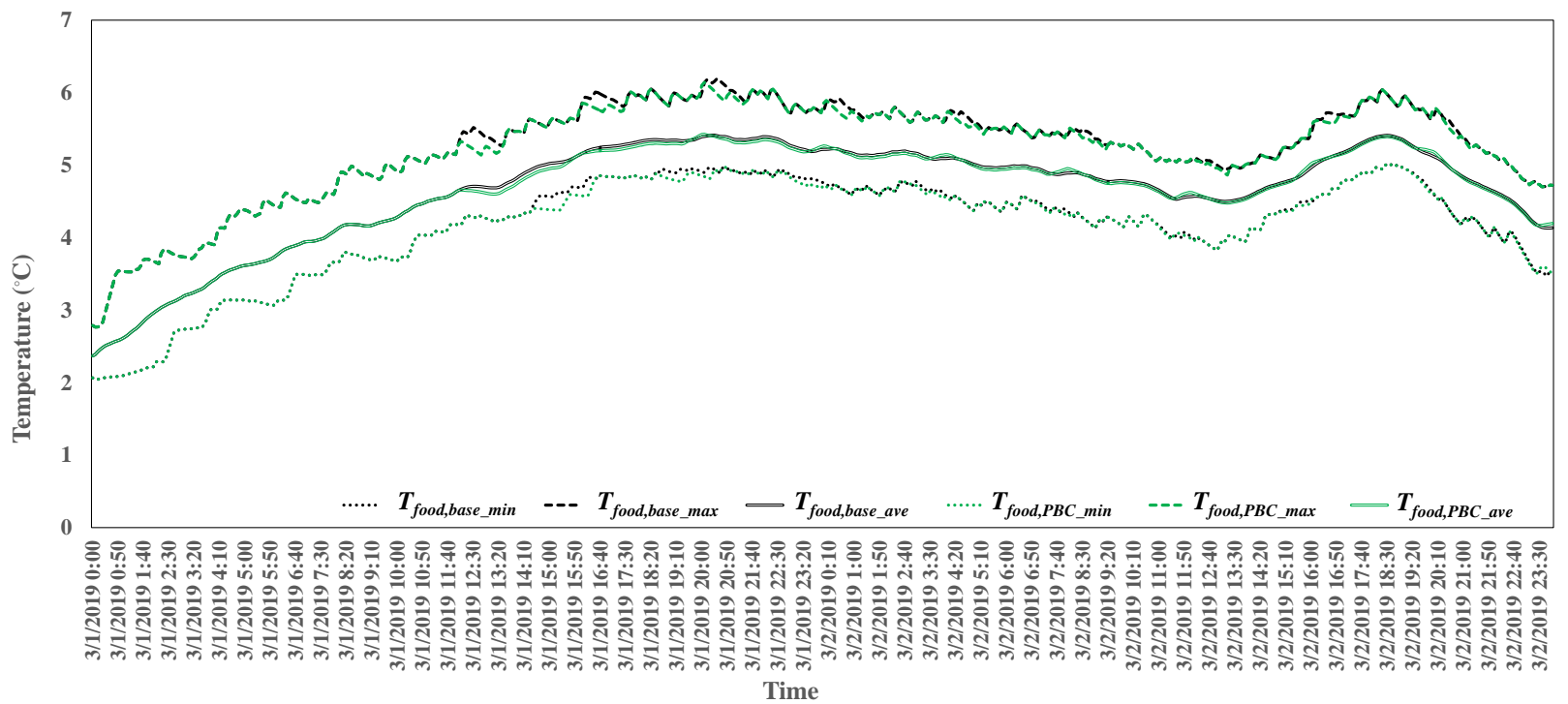

Figure 46: Frozen food temperature profiles for baseline and PBC with a load-up period of 12:00 p.m. to 1:00 p.m., March 2, 2019.

Figure 47and Figure 48 show the maximum allowable peak power reduction and energy reduction achieved in the hourly load-shed scenario. Both follow the 4-hour running/defrosting cycle pattern, as shown in Figure 40. Figure 49 shows the intuitive relationship among the maximum peak power reduction percentage, maximum energy reduction percentage, and the lowest targeted power limit in each hour. Maximum power reduction percentage and maximum energy reduction percentage follow a very similar pattern, which is not similar to that of baseline power consumption. This leads to the important finding that it is the cycling pattern of display cases, not the baseline total power consumption determined by outside weather conditions, that plays a decisive role in deciding the peak power reduction potential. The pattern of the lowest targeted power limit profile is reversed to that of the maximum power reduction percentage or the maximum energy reduction percentage profile: The limit is low when the percentage is high and vice versa. 


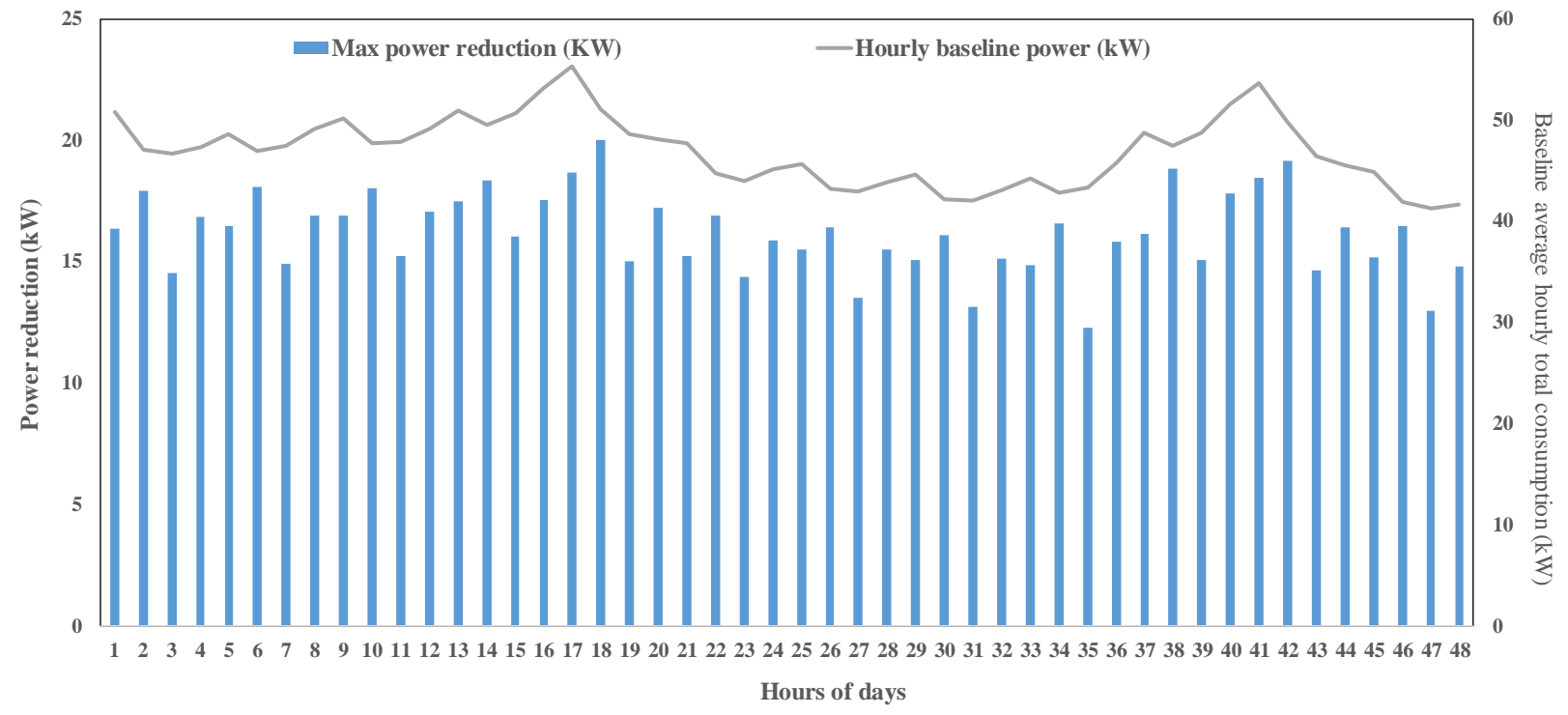

Figure 47: Power reduction achieved with PBC by hour of day for two days.

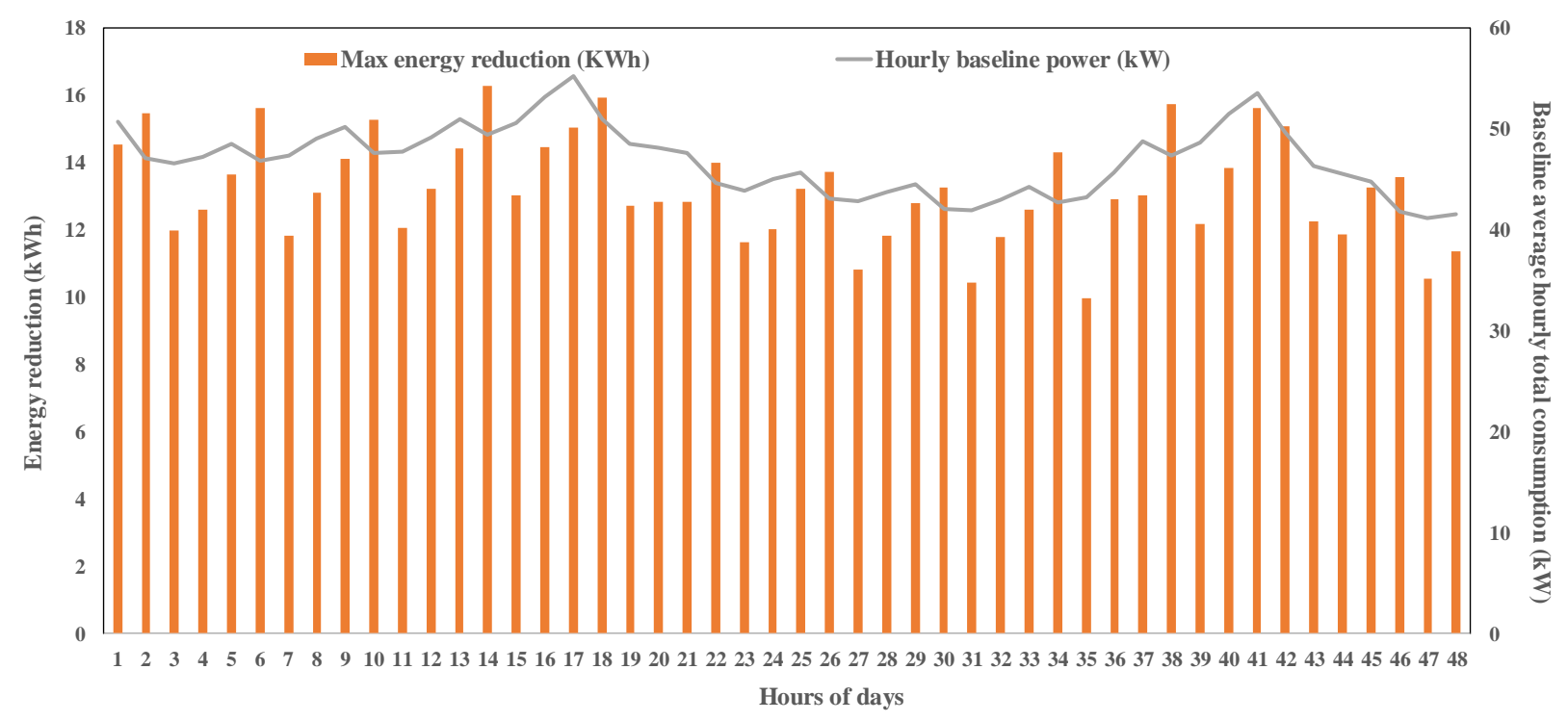

Figure 48: Energy reduction achieved with $\mathrm{PBC}$ by hour of day for two days. 


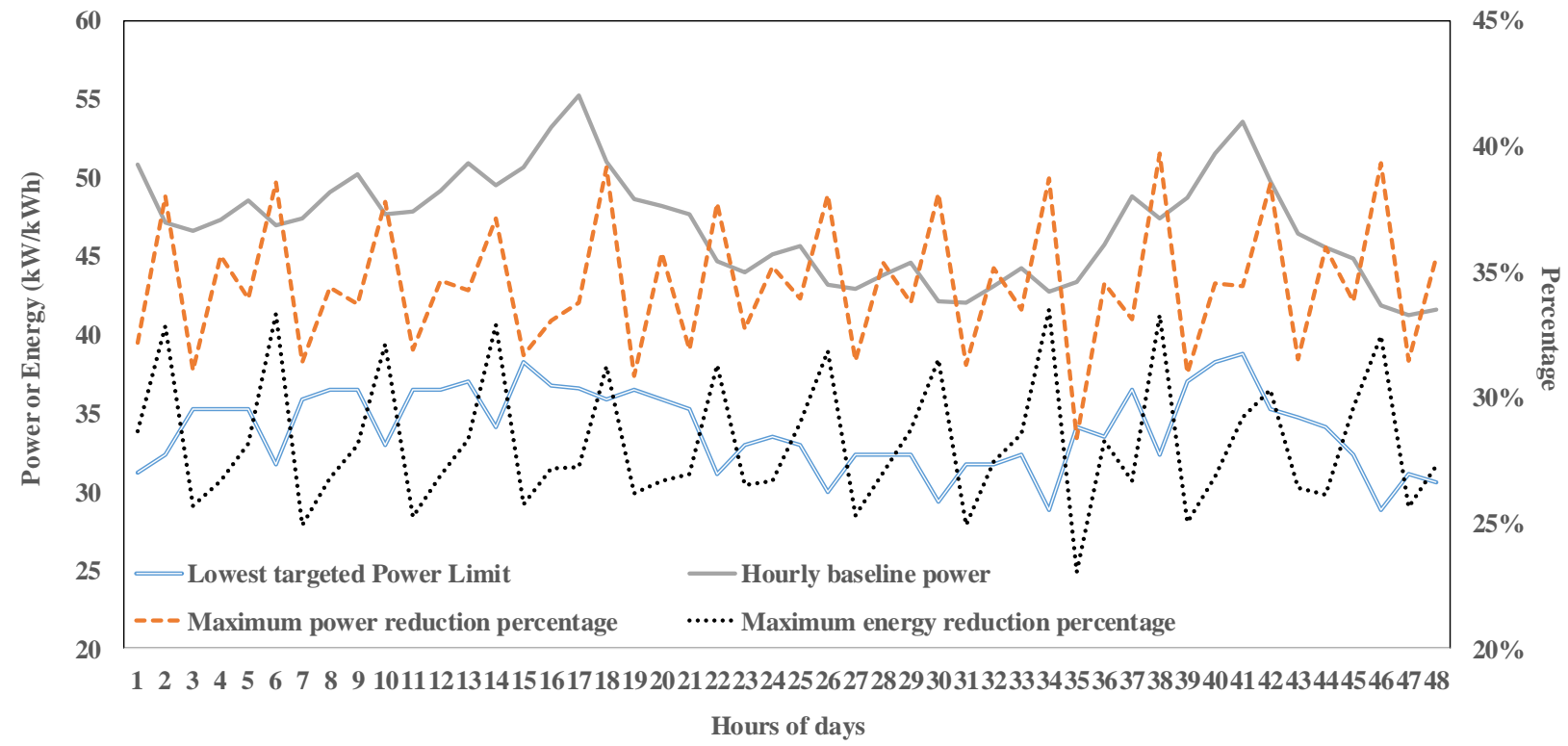

Figure 49: Power and energy reduction percentages in each hour for the load-shed scenario.

Figure 50 and Figure 51 show the maximum allowable peak power increase and energy increase achieved in the hourly load-up scenario. Compared with the reduction potentials from the load-shed scenario, it can be seen that the peak power and energy increase are much less. The maximum power increase percentage and the maximum energy increase percentage follow a very similar pattern, as shown in Figure 52.

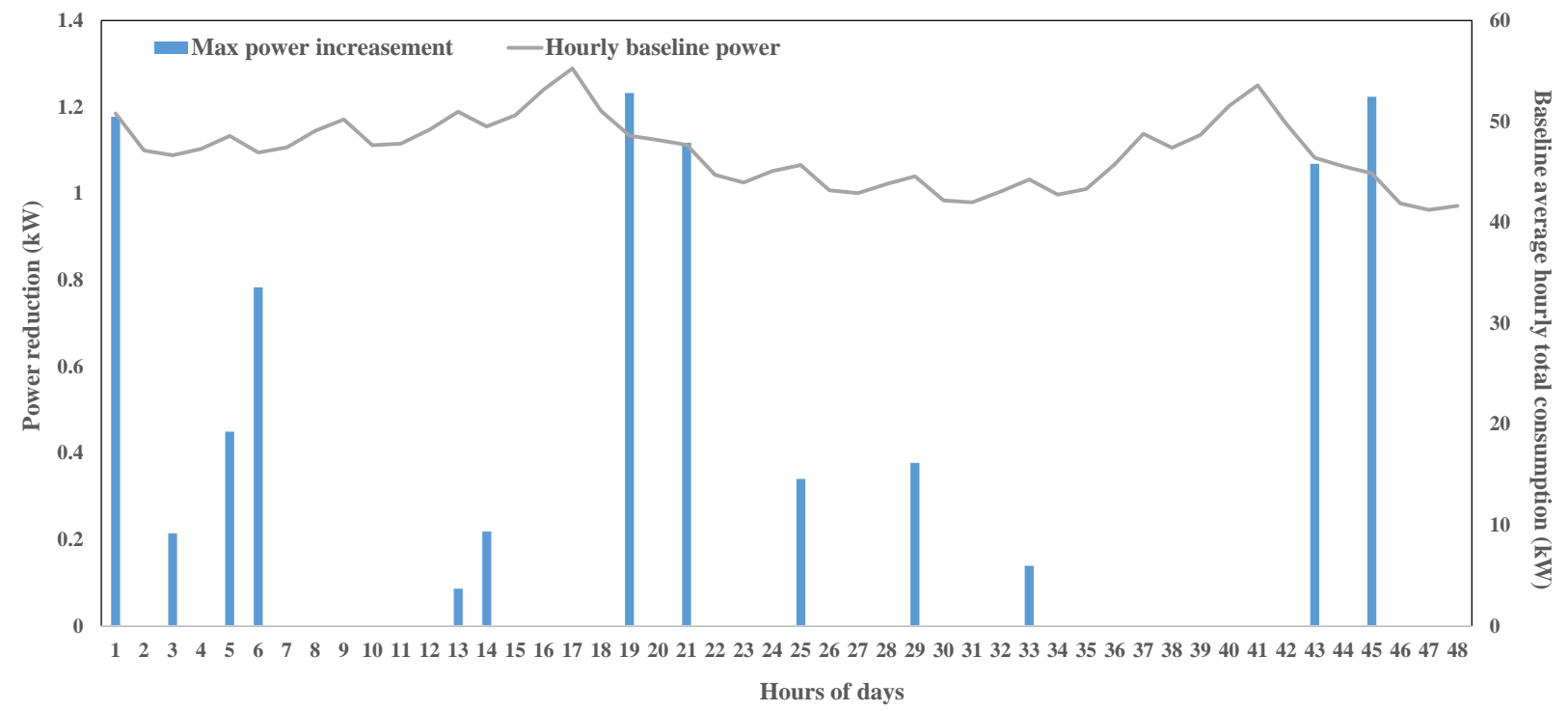

Figure 50: Power increase achieved with PBC by hour of day for two days. 


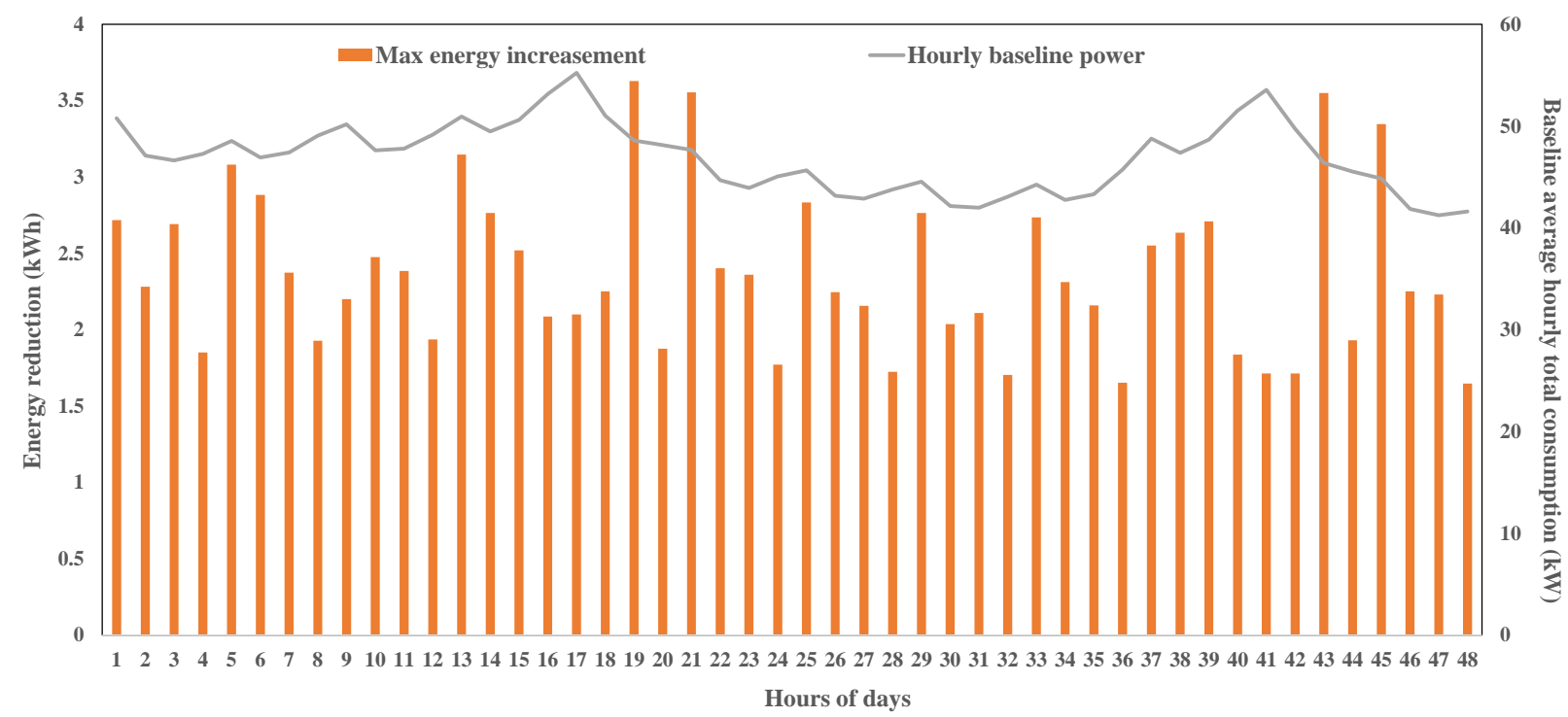

Figure 51: Energy increase achieved with PBC by hour of day for two days.

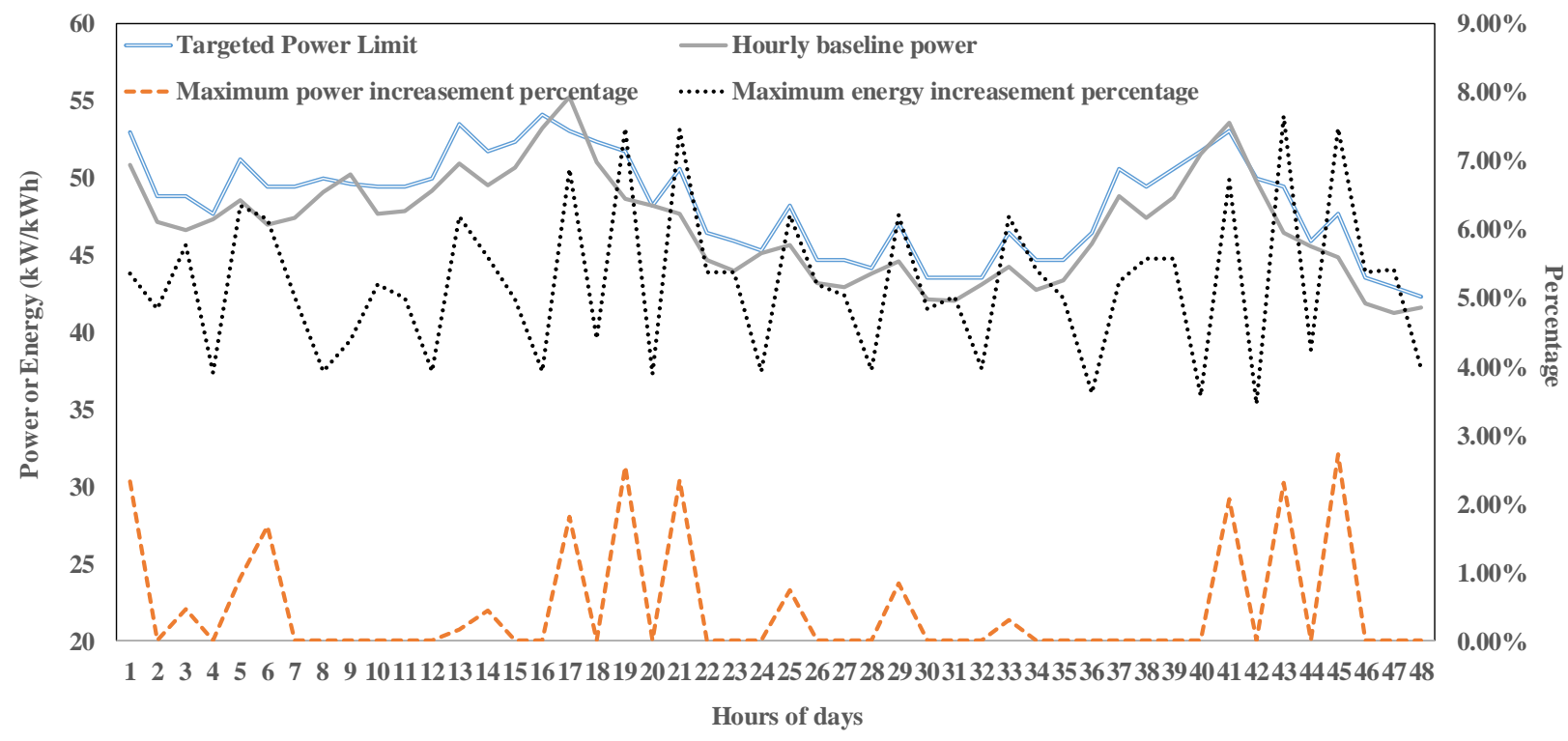

Figure 52: Power and energy reduction percentages in each hour for the load-up scenario.

\subsubsection{Discussion}

In this study, both an open-case refrigeration display case thermal model and simplified $\mathrm{CO}_{2}$ compressor model are developed with actual measured data. The validation results show that the developed models have satisfactory accuracies. The quantification of the hourly load flexibility is realized by PBC for four sets of compressors for all hours of two consecutive days. Each set includes two fixed-speed compressors and one variable-speed compressor. The total rated cooling capacity is $34,000 \mathrm{~W}(11,100+11,400+$ $11,300 \mathrm{~W})$. Each set corresponds to 12 display cases since the rated cooling capacity of each one is $2,810 \mathrm{~W}$.

The power reduction potential of refrigerators is related to the ambient temperature, initial statuses (e.g., defrost status, recover status, air-in-case temperature, food temperature, case temperature- and 
accumulated running time), and parameter values $\left(C_{f o o d}\right)$. There is no significant power peak for the baseline simulation with variations because of case defrost patterns having a similar magnitude due to changes in outdoor air temperature. If the outdoor air temperature had larger swings between day and night, this might not be the case.

Typical peak power reduction over the course of an hour does not vary significantly with time of day and is more closely tied to the defrost pattern of the cases. This value is $\sim 300 \mathrm{~W}$ per refrigerated case. Energy reduction potential for each hour is $\sim 250 \mathrm{Wh}$ per refrigerated case.

Peak power increase potential over an hour is not significant, and energy increase potential for an hour is also small, averaging less than $50 \mathrm{Wh}$ per case.

Note that the results of this study look at only one control mechanism - turning refrigeration on or off to the display cases. Given the complexity of refrigeration systems, there are many other control mechanisms that may yield increased power flexibility. These options are currently being investigated and include changing case set point temperatures, changing the compressor target suction saturation temperature, directly controlling the variable-speed compressor, directly controlling the fixed-speed compressors, and adjusting and coordinating the defrost schedules of the cases. 


\section{REFERENCES}

[1] U.S. DOE, 2015 Grid Modernization Multi-Year Program Plan, U.S. Department of Energy, 2015.

[2] U.S. EIA, "2015 Residential Energy Consumption Survey," Office of Energy Consumption and Efficiency Statistics, 2018.

[3] U.S. EIA, "2012 Commercial Buildings Energy Consumption Survey," U.S. Energy Information Agency, 2016.

[4] B. Cui, J. Munk, R. Jackson, D. Fugate, and M. Starke, "Building thermal model development of typical house in U.S. for virtual storage control of aggregated building loads based on limited available information," in 2017 ECOS Conference Proceedings, San Diego, CA, 2017.

[5] X. H. Xu and S. W. Wang, "A simplified dynamic model for existing buildings usign CTF and thermal network modles," International Journal of Thermal Sciences, pp. 1249-1262, 2008.

[6] U.S. DOE, "BEopt v 2.7," U.S. Department of Energy, 2016.

[7] U.S. EIA, "Electric Power Monthly with Data for February 2019," April 2019. https://www.eia.gov/electricity/monthly/archive/april2019.pdf.

[8] J. McLaren, "A Survey of U.S. Demand Charges," Clean Energy Group Webinar, September 19, 2017," https://www.nrel.gov/solar/assets/pdfs/2017-us-demand-charges-webinar.pdf.

[9] J. Dong, J. Munk, B. Cui, P. Boudreaux, and T. Kuruganti, "Machine-Learning Model of Electric Water Heater for Electricity Consumption Prediction," in 5th International High Performance Buildings Conference at Purdue, West Lafayette, IN, 2018. 
